April 1998

DFUB 98-7

Version 1

hep-th/9804043

\title{
BASIC AND EQUIVARIANT COHOMOLOGY IN BALANCED TOPOLOGICAL FIELD THEORY
}

\author{
by \\ Roberto Zucchini \\ Dipartimento di Fisica, Università degli Studi di Bologna \\ V. Irnerio 46, I-40126 Bologna, Italy \\ and \\ INFN, sezione di Bologna
}

\begin{abstract}
We present a detailed algebraic study of the $N=2$ cohomological set-up describing the balanced topological field theory of Dijkgraaf and Moore. We emphasize the role of $N=2$ topological supersymmetry and $\mathfrak{s l}(2, \mathbb{R})$ internal symmetry by a systematic use of superfield techniques and of an $\mathfrak{s l}(2, \mathbb{R})$ covariant formalism. We provide a definition of $N=2$ basic and equivariant cohomology, generalizing Dijkgraaf's and Moore's, and of $N=2$ connection. For a general manifold with a group action, we show that: $i$ ) the $N=2$ basic cohomology is isomorphic to the tensor product of the ordinary $N=1$ basic cohomology and a universal $\mathfrak{s l}(2, \mathbb{R})$ group theoretic factor: $i i)$ the affine spaces of $N=2$ and $N=1$ connections are isomorphic.
\end{abstract}

PACS no.: 0240, 0460, 1110. Keywords: Topological Field Theory, Cohomology. 


\section{Introduction}

Topological quantum field theories are complicated often fully interacting local renormalizable field theories, yet they can be solved exactly and the solution is highly non trivial. Expectation values of topological observables provide topological invariants of the manifolds on which the fields propagate. These invariants are independent from the couplings and to a large extent from the interactions between the fields. At the same time, topological field theories are often topological sectors of ordinary field theories. In this way, they are convenient testing grounds for subtle non perturbative field theoretic phenomena. See f. i. refs. [1-3] for an updated comprehensive review on the subject and complete referencing.

$N=1$ cohomological topological field theories have been the object of intense and exhaustive study. They can be understood in the framework of equivariant cohomology of infinite dimensional vector bundles [4-9] and realized as Mathai-Quillen integral representations of Euler classes [10-13]. The resulting formalism is elegant and general and covers the important case where the quotient by the action of a gauge symmetry group is required. Each of these models describes the differential topology of a certain moduli space, depending on the model considered: the field theoretic correlation functions of topological observables correspond to intersection numbers on the moduli space.

$N=2$ cohomological topological field theories were discovered quite early [14-17], but they did not arouse much interest until recently when it became clear that they might provide important clues in the analysis of $S$ duality in supersymmetric Yang-Mills theory and in the study of the world volume theories of $D$-branes in string theory.

In ref. [18], Vafa and Witten performed an exact strong coupling test of $S$ duality of $N=4$ supersymmetric 4-dimensional Yang-Mills theory by studying a topologically twist of the model yielding an $N=2$ cohomological field theory. They showed that the partition function is $Z(\tau)=\sum_{k} a_{k} \exp (2 \pi i \tau k)$, where $a_{k}$ is the Euler characteristic of the moduli space of $k$ instantons, and tested $S$ duality by analyzing the modularity properties of $Z(\tau)$. Their work, inspired by the original work of Yamron [14], was soon developed and refined in a series of papers [19-24]. In ref. [25], Bershadsky, Sadov and Vafa showed that the three $N=2$ cohomological topological field theories obtained by the non topological twistings of $N=4$ supersymmetric 4-dimensional Yang-Mills theory arose from curved 3-branes embedded in Calabi-Yau manifolds and manifolds with exceptional holonomy groups. Their analysis was continued and further developed in refs. [21,26-28], where the connection with higher dimensional instantons was elucidated. In ref. [29], Park constructed a family of Yang-Mills instantons from $D$-instantons in topological twisted 
$N=4$ supersymmetric 4-dimensional Yang-Mills theory. In ref. [30], Hofman and Park worked out a 2 -dimensional $N=2$ cohomological topological field theory as a candidate for covariant second quantized RNS superstrings, which they conjectured to be a formulation of $M$ theory.

All the endeavors mentioned above, and many other related ones, which we cannot mention for lack of space, show that $N=2$ cohomological topological field theories are relevant in a variety of physical and mathematical issues. In spite of that, the body of literature devoted to the study of the geometry of such models is comparatively small. In ref. [17], Blau and Thompson worked out a Riemannian formulation of $N=2$ topological gauge theory using $N=2$ topological superfield techniques. In ref. [31], Dijkgraaf and Moore showed that all known $N=2$ topological models were examples of "balanced topological field theories" and developed a cohomological framework suitable for their study. In ref. [21], Blau and Thompson proved the equivalence of their earlier formulation and Dijkgraaf's and Moore's. These studies show that the partition function of every $N=2$ topological model calculates the Euler characteristic of some moduli space of vanishing virtual dimension. They also indicate that the appropriate cohomological scheme is provided by $N=2$ basic or equivariant cohomology. The present paper aims at a systematic study of the latter developing the ideas of [31].

In general, a cohomological topological field theory is characterized by a symmetry Lie algebra $\mathfrak{g}$, a graded algebra of fields $f$ and a set of graded derivations on $f$ generating a Lie algebra $t$. In turn, the topological algebra $t$ provides the algebraic and geometric framework for the definition of the topological observables [1].

As is well-known, in $N=1$ cohomological topological field theory, $\mathrm{t}$ is generated by four derivations $k, d, j(\xi), l(\xi), \xi \in \mathfrak{g}$, of degrees $0,1,-1,0$, respectively, obeying the graded commutation relations $(2.1 .14),(2.1 .15 \mathrm{a})-(2.1 .15 \mathrm{c}),(2.1 .16),(2.1 .17 \mathrm{a})-(2.1 .17 \mathrm{~b})$ and $(2.1 .18 \mathrm{a})-(2.1 .18 \mathrm{c})$ below. $k$ is the ghost number operator. $d$ is the nilpotent topological charge. $j(\xi), l(\xi)$ describe the action of the symmetry Lie algebra $\mathfrak{g}$ on fields. The elements $\alpha \in \mathrm{f}$ are classified into the eigenspaces $\mathrm{f}^{p}, p \in \mathbb{Z}$, of $k$. The $N=1$ basic degree $p$ cohomology of $\mathrm{f}$ is defined by

$$
\begin{aligned}
& j(\xi) \alpha=0, \quad l(\xi) \alpha=0, \quad \xi \in \mathfrak{g}, \\
& d \alpha=0, \quad \alpha \equiv \alpha+d \beta, \quad \beta \in \mathrm{f}^{p-1}, \quad \alpha \in \mathfrak{f}^{p} .
\end{aligned}
$$

The $N=1$ Weil algebra w, an essential element of the definition of the $N=1$ equivariant cohomology of $\mathfrak{f}$, is generated by two $\mathfrak{g}$ valued fields $\omega$, $\phi$ of degrees 1,2 , respectively. $t$ acts on $w$ according to $(3.1 .10 \mathrm{a}),(3.1 .10 \mathrm{~b}),(3.1 .11 \mathrm{a}),(3.1 .11 \mathrm{~b}),(3.1 .12 \mathrm{a})-$ (3.1.12d) below. 
$k, d, j(\xi), l(\xi)$ can be organized into two $N=1$ topological superderivation

$$
\begin{aligned}
H & =k-\theta d, \\
I(\xi) & =j(\xi)+\theta l(\xi), \quad \xi \in \mathfrak{g} .
\end{aligned}
$$

The Lie algebra structure of $\mathrm{t}$ is compatible with the underlying $N=1$ topological supersymmetry, since the relevant commutation relations can be written in terms of the superderivations $H, I(\xi)$. Similarly, $\omega, \phi$ can be organized into the $\mathfrak{g}$ valued superfield

$$
W=\omega+\theta\left(\phi-\frac{1}{2}[\omega, \omega]\right) .
$$

The action of $\mathrm{t}$ on $\mathrm{w}$ can be written in terms of the superderivations $H, I(\xi)$ and the superfield $W$ in a manifestly $N=1$ supersymmetric way.

Analogously, in $N=2$ cohomological topological field theory, $\mathrm{t}$ is generated by seven graded derivations $u_{A}, A=1,2, t_{A B}, A, B=1,2$, symmetric in $A, B, k, d_{A}, A=1,2, j(\xi)$, $j_{A}(\xi), A=1,2, l(\xi), \xi \in \mathfrak{g}$, of degrees $-1,0,0,1,-2,-1,0$, respectively, obeying the graded commutation relations $(2.2 .16 \mathrm{a})-(2.2 .16 \mathrm{c}),(2.2 .17 \mathrm{a})-(2.2 .17 \mathrm{j}),(2.2 .18 \mathrm{a})-(2.2 .18 \mathrm{c})$, (2.2.19a)-(2.2.19f), (2.2.20a)-(2.2.20f) below. The $u_{A}$ are a sort of homotopy operators and constrain the cohomology of $\mathrm{f}$, defined shortly, to an important extent. The $t_{A B}$ and $k$ are the generators of the internal $\mathfrak{s l}(2, \mathbb{R}) \oplus \mathbb{R}$ symmetry Lie algebra of $\mathrm{t}$. The $d_{A}$ are the nilpotent topological charges. $j(\xi), j_{A}(\xi), l(\xi)$ describe the action of the symmetry Lie algebra $\mathfrak{g}$ on fields. The elements $\alpha \in \mathrm{f}$ are classified into the eigenspaces $\mathrm{f}^{n, p}, n \in \mathbb{N}$, $p \in \mathbb{Z}$, of the invariants $c, k$ of the internal algebra $\mathfrak{s t}(2, \mathbb{R}) \oplus \mathbb{R}$. The $N=2$ basic type $n, p$ cohomology of $f$ is defined by

$$
\begin{aligned}
& j(\xi) \alpha=0, \quad j_{A}(\xi) \alpha=0, \quad l(\xi) \alpha=0, \quad \xi \in \mathfrak{g}, \\
& d_{A} \alpha=0, \quad \alpha \equiv \alpha+\frac{1}{2} \epsilon^{K L} d_{K} d_{L} \beta, \quad \beta \in \mathrm{f}^{n, p-2}, \quad \alpha \in \mathrm{f}^{n, p} .
\end{aligned}
$$

It is possible to show, using the basic relation $\left[d_{A}, u_{B}\right]=\frac{1}{2}\left(t_{A B}+\epsilon_{A B} k\right)$, that this cohomology is trivial for $p \neq \pm n+1$.

The $N=2$ Weil algebra $\mathrm{w}$, entering the definition of $N=2$ equivariant cohomology, is generated by four $\mathfrak{g}$ valued fields $\omega_{A}, A=1,2, \phi_{A B}, A, B=1,2$, symmetric in $A, B, \gamma, \rho_{A}$, $A=1,2$, of degrees $1,2,2,3$, respectively. $\mathrm{t}$ acts on $\mathrm{w}$ according to $(3.2 .10 \mathrm{a})-(3.2 .10 \mathrm{~h})$, (3.2.11a)-(3.2.11h), (3.2.12a)-(3.2.12l).

$u_{A}, t_{A B}, k, d_{A}, j(\xi), j_{A}(\xi), l(\xi)$ can be organized into two $N=2$ topological superderivation

$$
\begin{aligned}
H_{A} & =u_{A}+\frac{1}{2} \theta^{K}\left(t_{A K}-\epsilon_{A K} k\right)-\frac{1}{2} \epsilon_{K L} \theta^{K} \theta^{L} d_{A}, \\
I(\xi) & =j(\xi)+\theta^{K} j_{K}(\xi)+\frac{1}{2} \epsilon_{K L} \theta^{K} \theta^{L} l(\xi), \quad \xi \in \mathfrak{g} .
\end{aligned}
$$


The Lie algebra structure of $\mathrm{t}$ is compatible with the underlying $N=2$ topological supersymmetry, since the relevant commutation relations can be written in terms of the superderivations $H_{A}, I(\xi)$. Similarly, $\omega_{A}, \phi_{A B}, \gamma, \rho_{A}$ can be organized into the $\mathfrak{g}$ valued superfield

$$
\begin{aligned}
W & =\omega_{A}+\theta^{K}\left(\phi_{A K}+\epsilon_{A K} \gamma-\frac{1}{2}\left[\omega_{A}, \omega_{K}\right]\right)+\frac{1}{2} \epsilon_{M N} \theta^{M} \theta^{N}\left(-2 \rho_{A}-\epsilon^{K L}\left[\omega_{K}, \phi_{A L}\right]\right. \\
& \left.+\left[\omega_{A}, \gamma\right]+\frac{1}{6} \epsilon^{K L}\left[\omega_{K},\left[\omega_{L}, \omega_{A}\right]\right]\right) .
\end{aligned}
$$

The action of $\mathrm{t}$ on $\mathrm{w}$ can be written in terms of the superderivations $H_{A}, I(\xi)$ and the superfield $W$ in a manifestly $N=2$ supersymmetric way.

In the first part of this paper, we study the topological algebra $t$ and the Weil algebra w abstractly both in the $N=1$ and in the $N=2$ case. We show that their structure is essentially dictated by rather general requirements of closure and topological supersymmetry, called stability conditions, which can be defined for any value of $N$. In this way, we show that all these algebraic structures can be derived from a single universal model independent notion and also suggest an obvious method for generalizing them to higher values of $N$. In the second part of the paper, we define basic and equivariant cohomology, abstract connections and the Weil homomorphism both in the $N=1$ and in the $N=2$ case and study some of their properties. Finally, in the third part of the paper, we study the cohomology of manifolds carrying a right group action and show that, in this important case, the $N=2$ type $(k, k+1)$ basic cohomology is isomorphic to the tensor product of the $N=1$ degree $k$ basic cohomology and the completely symmetric tensor space $\bigvee^{k-1} \mathbb{R}^{2}$ and that the affine spaces of $N=2$ and $N=1$ connections are isomorphic.

Throughout the paper, we stress the role of topological supersymmetry, also because we feel that, on this score, confusing claims have appeared in the literature. This has allowed us to discover the derivations $u_{A}$ and $k$ introduced above, which are not mentioned in ref. [31], but which are required by $N=2$ topological supersymmetry and constrain structurally the $N=2$ cohomology.

The definition of $N=2$ basic cohomology given above is more general than that used in ref. [31], which is limited to the important case where $n=1$. In our judgement, this definition is more appropriate, yielding the aforementioned fundamental relation between the $N=1$ and $N=2$ basic cohomologies of manifolds with a right group action.

This paper is organized as follows. In sect. 1, we introduce and precisely define the crucial notion of stability. In sect. 2 , we show that both the $N=1$ and $N=2$ topological algebras can be derived in a model independent way by imposing non degeneracy and stability. In sect. 3, we show similarly that non degeneracy and stability allow to obtain 
in abstract fashion both the $N=1$ and $N=2$ Weil algebra. In sect. 4, we define the relevant notions of $N=1$ and $N=2$ basic cohomology, highlighting their similarities and differences. In sect. 5, we study the Weil superoperation and its cohomology. In sect. 6, we define $N=1$ and $N=2$ abstract connections, equivariant cohomology and the related Weil homomorphism. In sect. 7, we apply our algebraic set up to study the cohomology of manifolds carrying a right group action and work out the relation between $N=1$ and $N=2$ cohomology. Finally, sect. 8 outlines future lines of inquiry.

\section{Superalgebras, supermodules and stability}

\section{1. $\mathbb{Z}$ graded algebras and the corresponding superalgebras}

All the vector spaces, algebras and modules considered in this section are real and $\mathbb{Z}$ graded. If $\mathrm{s}$ is such a space, we denote by $\mathrm{s}^{k}$ the subspace of $\mathrm{s}$ of degree $k \in \mathbb{Z}$. If $\mathrm{s}=\mathrm{s}^{0}, \mathrm{~s}$ is called ungraded.

Let $N \in \mathbb{N}$. Let $\theta^{A}, A=1, \cdots, N$, be a $N$-uple of Grassmann odd generators which are conventionally assigned degree -1 :

$$
\theta^{A} \theta^{B}+\theta^{B} \theta^{A}=0, \quad A, B=1, \cdots, N ; \quad \operatorname{deg} \theta^{A}=-1, \quad A=1, \cdots, N .
$$

The $\theta^{A}$ generate a Grassmann algebra $\Lambda_{N}[\theta]$.

Let a be a $\mathbb{Z}$ graded algebra. The $N$ superalgebra $\mathrm{A}_{N}$ associated to a is the graded tensor product algebra

$$
\mathrm{A}_{N}=\Lambda_{N}[\theta] \hat{\otimes} \mathbf{a}
$$

with the canonical $\mathbb{Z}$ grading.

\subsection{Stability}

In this subsection, we introduce the notion of stability, which will play an important role throughout this paper.

Let $\mathrm{u}$ and a be an ungraded real vector space and a $\mathbb{Z}$ graded real algebra, respectively. Let $\mathcal{T}=\left\{T_{r} \mid r \in R\right\}$ be a finite subset of $\operatorname{Hom}\left(\mathrm{u}, \mathrm{A}_{N}\right)$.

Definition 1.2.1. $\mathcal{T}$ is called stable if:

i) for $r \in R$, there is $p_{r}^{\mathcal{T}} \in \mathbb{Z}$ such that, for all $x \in \mathrm{u}, T_{r}(x) \in \mathrm{A}_{N}^{p^{\mathcal{T}}{ }^{r}}$;

ii) for $x \in \mathrm{u}, x \neq 0$, the $T_{r}(x)$ are linearly independent;

iii) there are constants $k^{\mathcal{T}} A r{ }^{s} \in \mathbb{R}, r, s \in R, A=1, \cdots, N$, such that, for $x \in \mathrm{u}$,

$$
\partial_{A} T_{r}(x)=k_{A r}^{\mathcal{T}}{ }^{s} T_{s}(x), \quad r \in R, A=1, \cdots, N
$$


where $\partial_{A}=\partial / \partial \theta^{A}$.

If $\mathrm{u}$ is an algebra, it is also required that:

iv) there are constants $g^{\mathcal{T}}{ }_{r s}{ }^{t} \in \mathbb{R}, r, s, t \in R$, such that, for $x, y \in \mathrm{u}$,

$$
T_{r}(x) T_{s}(y)=g_{r s}^{\mathcal{T}} T_{t}^{t}(x y), \quad r, s \in R .
$$

For $x \in \mathrm{u}$, denote by $\mathrm{T}(x)$ the linear span of the $T_{r}(x)$. Then, by the above, $\mathrm{T}(x)$ is stable under $\partial_{A}$. Further, when $\mathrm{u}$ is an algebra, $\mathrm{T}(x) \mathrm{T}(y) \subseteq \mathrm{T}(x y)$.

The following remarks are in order.

a) By $i i$ above, if $\mathrm{u} \neq 0$, the constants $k^{\mathcal{T}}{ }_{A r}{ }^{s}$ are uniquely determined; further, when $\mathrm{u}$ is an algebra and $\mathbf{u u} \neq 0$, the $g^{\mathcal{T}}{ }_{r s}^{t}$, also, are uniquely determined.

b) By grading reasons, when $u \neq 0$,

$$
k^{\mathcal{T}}{ }_{A r}{ }^{s}=0, \quad \text { unless } p^{\mathcal{T}}{ }_{r}-p^{\mathcal{T}}{ }_{s}+1=0 ;
$$

further, when $u$ is an algebra such that $u u \neq 0$,

$$
g^{\mathcal{T}}{ }_{r s}^{t}=0, \quad \text { unless } p^{\mathcal{T}}{ }_{r}+p^{\mathcal{T}}{ }_{s}-p^{\mathcal{T}}{ }_{t}=0 .
$$

c) As $\partial_{A} \partial_{B}+\partial_{B} \partial_{A}=0$, one has

$$
k^{\mathcal{T}}{ }_{A r}{ }^{t} k^{\mathcal{T}}{ }_{B t}{ }^{s}+k^{\mathcal{T}}{ }_{B r}{ }^{t} k^{\mathcal{T}}{ }_{A t}{ }^{s}=0,
$$

when $\mathbf{u} \neq 0$; further, if $\mathbf{u}$ is an algebra, being $\partial_{A} T_{r}(x) T_{s}(y)+(-1)^{p^{\tau}{ }_{r}} T_{r}(x) \partial_{A} T_{s}(y)=$ $g^{\mathcal{T}}{ }_{r s}{ }^{t} \partial_{A} T_{t}(x y)$ by (1.2.2), one has further

$$
k^{\mathcal{T}}{ }_{A r}{ }^{u} g^{\mathcal{T}}{ }_{u s}{ }^{t}+(-1)^{p^{\mathcal{T}}{ }_{r}} k^{\mathcal{T}}{ }_{A s}{ }^{u} g^{\mathcal{T}}{ }_{r u}{ }^{t}=g^{\mathcal{T}}{ }_{r s}{ }^{u} k^{\mathcal{T}}{ }_{A u}{ }^{t},
$$

when $u u \neq 0$.

d) If $\mathrm{u}$ is an algebra, there are further constraints on the $g^{\mathcal{T}}{ }_{r s}{ }^{t}$ coming from such properties as associativity, Jacobi relations, etc., when they hold.

Usually, the constraints (1.2.3)-(1.2.6), are so stringent that, given some extra non singularity assumptions, allow the determination of the $k^{\mathcal{T}} A r^{s}$ and $g^{\mathcal{T}}{ }_{r s}^{t}$, up to the natural equivalence relation associated to the non singular linear redefinition of the $T_{r}$.

Let a be $\mathbb{Z}$ graded real algebra. Let $\mathcal{H}=\left\{H_{r} \mid r \in R\right\}$ be a finite subset of $\mathrm{A}_{N}$.

Definition 1.2.2. $\mathcal{H}$ is called stable, if, defining

$$
T^{\mathcal{H}}{ }_{r}(x)=x H_{r}, \quad x \in \mathbb{R},
$$

$\mathcal{T}^{\mathcal{H}}=\left\{T^{\mathcal{H}}{ }_{r} \mid r \in R\right\}$ is a stable subset of $\operatorname{Hom}\left(\mathbb{R}, \mathrm{A}_{N}\right)$ as defined above. 
We denote by $\mathrm{H}$ the linear span of the $H_{r}$. Then, $\mathrm{H}$ is a subalgebra of $\mathrm{A}_{N}$.

Let $\mathrm{u}, \mathrm{a}$, e, q be an ungraded real algebra, a $\mathbb{Z}$ graded real algebra, an ungraded real left $\mathrm{u}$ module and a $\mathbb{Z}$ graded real left a module algebra, respectively. Let $\mathcal{T}=\left\{T_{r} \mid r \in R\right\}$, $\mathcal{M}=\left\{M_{i} \mid i \in I\right\}$ be stable subsets of $\operatorname{Hom}\left(\mathrm{u}, \mathrm{A}_{N}\right)$ and $\operatorname{Hom}\left(\mathrm{e}, \mathrm{Q}_{N}\right)$, respectively. Let $C \in \mathrm{Q}_{N}^{0}$ such that $\partial_{A} C=0$.

Definition 1.2.3. We say that $\mathcal{T}$ acts $C$-stably on $\mathcal{M}$ on the left if:

i) if $C \neq 0$, then for $m \in \mathrm{e}, C$ is linearly independent from the $M_{i}(m)$;

ii) for $x \in \mathrm{u}$,

$$
T_{r}(x) C=0 ;
$$

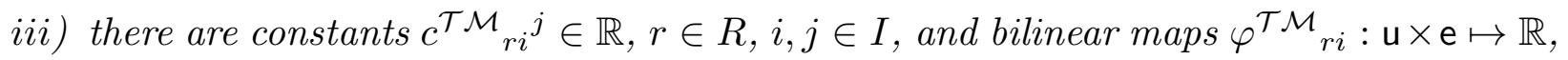
$r \in R, i \in I$ such that, for any $x \in \mathrm{u}$ and $m \in \mathrm{e}$,

$$
T_{r}(x) M_{i}(m)=c^{\mathcal{T} \mathcal{M}_{r i}{ }^{j} M_{j}(x m)+\varphi^{\mathcal{T} \mathcal{M}}}{ }_{r i}(x, m) C, \quad r \in R, i \in I .
$$

When $C=0$ above, we say that $\mathcal{T}$ acts stably on $\mathcal{M}$ on the left.

So, for any $x \in \mathrm{u}$ and $m \in \mathrm{e}, \mathrm{T}(x) \mathrm{M}(m) \subseteq \mathrm{M}(x m) \oplus \mathbb{R} C$.

Note the following.

a) By the above definition, if ue $\neq 0$, the constants $c^{\mathcal{M T}}{ }_{r i}{ }^{j}$ are uniquely determined; also, if $C \neq 0$, the maps $\varphi^{\mathcal{T} \mathcal{M}_{r i}}$ are uniquely determined.

b) By grading reasons, when ue $\neq 0$,

$$
c^{\mathcal{T} \mathcal{M}_{r i}{ }^{j}}=0, \quad \text { unless } p_{r}^{\mathcal{T}}+p^{\mathcal{M}}{ }_{i}-p^{\mathcal{M}}{ }_{j}=0
$$

similarly, when $C \neq 0$,

$$
\varphi^{\mathcal{T} \mathcal{M}_{r i}(x, m)}=0, \quad \text { unless } p_{r}^{\mathcal{T}}+p^{\mathcal{M}}{ }_{i}=0 .
$$

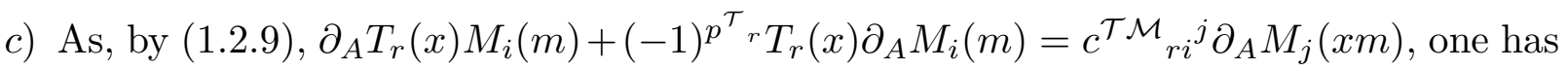

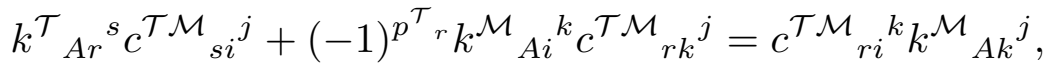

when ue $\neq 0$; further, as $\left(T_{r}(x) T_{s}(y)\right) M_{i}(m)=T_{r}(x)\left(T_{s}(y) M_{i}(m)\right)$,

$$
\begin{aligned}
& g^{\mathcal{T}}{ }_{r s}{ }^{t} c^{\mathcal{T} \mathcal{M}_{t i}{ }^{j}}-c^{\mathcal{T} \mathcal{M}_{s i}{ }^{k}} c^{\mathcal{T} \mathcal{M}}{ }_{r k}{ }^{j}=0, \\
& g^{\mathcal{T}}{ }_{r s}{ }^{t} \varphi^{\mathcal{T} \mathcal{M}_{t i}(x y, m)-c^{\mathcal{T M}}{ }_{s i}{ }^{j} \varphi^{\mathcal{T} \mathcal{M}}}{ }_{r j}(x, y m)=0,
\end{aligned}
$$

when ue $\neq 0, C \neq 0$, respectively.

Usually, the constraints (1.2.10)-(1.2.14), are so stringent that, given some extra non singularity assumptions, allow the determination of the $c^{\mathcal{T} \mathcal{M}}{ }_{r i}{ }^{j}$ and $\varphi^{\mathcal{T M}}{ }_{r i}$, up to the natural equivalence relation associated to the non singular linear redefinition of the $T_{r}$ and $M_{i}$.

Let a, e, q be an ungraded real algebra, an ungraded real vector space and a $\mathbb{Z}$ graded real left a module algebra, respectively. Let $\mathcal{H}=\left\{H_{r} \mid r \in R\right\}, \mathcal{M}=\left\{M_{i} \mid i \in I\right\}$ be stable subsets of $A_{N}$ and $\operatorname{Hom}\left(e, Q_{N}\right)$, respectively. 
Definition 1.2.4. We say that $\mathcal{H}$ acts stably on $\mathcal{M}$, if $\mathcal{T}^{\mathcal{H}}$ (cfr. def. 1.2.2) acts stably on $\mathcal{M}$ as defined above, where e has the structure of $\mathbb{R}$ module corresponding to that of real vector space.

\subsection{The $N=1,2$ cases}

In this paper, we concentrate on the cases $N=1,2$. In this subsection, we introduce notation suitable for these special $N$ values.

Let a be a $\mathbb{Z}$ graded real algebra.

Let $N=1$. In this case, one can set $\theta^{1}=\theta$ for simplicity. If $X \in \mathrm{A}_{1}^{p}$ for some $p \in \mathbb{Z}$, then $X$ is of the form

$$
X=x+\theta \tilde{x}
$$

with $x \in \mathrm{a}^{p}$ and $\tilde{x} \in \mathrm{a}^{p+1}$. Note that

$$
x=\left.X\right|_{\theta=0} .
$$

Denoting $\partial=\partial / \partial \theta$, we define

$$
\tilde{X}=\partial X
$$

Clearly, $\tilde{X} \in \mathrm{A}_{1}^{p+1}$. Indeed,

$$
\tilde{X}=\tilde{x}
$$

Let $N=2$. If $X \in \mathrm{A}_{2}^{p}$ for some $p \in \mathbb{Z}$, then $X$ is of the form

$$
X=x+\theta^{A} x_{, A}+\frac{1}{2} \epsilon_{K L} \theta^{K} \theta^{L} \tilde{x}
$$

with $x \in \mathrm{a}^{p}, x_{, A} \in \mathrm{a}^{p+1}$ and $\tilde{x} \in \mathrm{a}^{p+2} \quad 1$. Note that

$$
x=\left.X\right|_{\theta=0} .
$$

Denoting $\partial_{A}=\partial / \partial \theta^{A}$, we define

$$
X_{, A}=\partial_{A} X
$$

Clearly, $X_{, A} \in \mathrm{A}_{2}^{p+1}$. Indeed,

$$
X_{, A}=x_{, A}+\epsilon_{A K} \theta^{K} \tilde{x}
$$

So,

$$
x_{, A}=\left.X_{, A}\right|_{\theta=0} \cdot
$$

1 The totally antisymmetric symbols $\epsilon_{A B}, \epsilon^{A B}$ are normalized so that $\left|\epsilon_{12}\right|=\left|\epsilon^{12}\right|=1$ and $\epsilon^{A K} \epsilon_{K B}=\epsilon_{B K} \epsilon^{K A}=\delta_{B}^{A}$. 
Finally, we set

$$
\tilde{X}=\frac{1}{2} \epsilon^{K L} \partial_{K} \partial_{L} X .
$$

Clearly, $\tilde{X} \in \mathrm{A}_{2}^{p+2}$, as

$$
\tilde{X}=\tilde{x} .
$$

The analysis of stability simplify considerably in the $N=1,2$ cases. In the $N=1$ case, stability is an almost trivial notion. For $N=2$, this is no longer so. The notion of stability is nevertheless useful, since it shows quite clearly in what sense several $N=2$ algebraic structures generalize their $N=1$ counterparts.

\section{Fundamental superstructures}

Let $d$ be a $\mathbb{Z}$ graded real Lie algebra, i.e. a $\mathbb{Z}$ graded real algebra whose product is graded antisymmetric. Let $\mathfrak{g}$ be a real ungraded Lie algebra.

\subsection{The fundamental $N=1$ superstructure}

Proposition 2.1.1. Let $H \in \mathrm{D}_{1}^{0}$. Assume that the set $\{H, \tilde{H}\}$ is stable (cfr. def. 1.2.2). Let $\mathrm{H}$ the linear span of this set. Assume that

$$
\left[\mathrm{H}^{0}, \mathrm{H}^{1}\right]=\mathrm{H}^{1} .
$$

Then, perhaps after substituting $H$ by $\kappa H$ for some suitable $\kappa \in \mathbb{R}_{\times}$, one has

$$
\begin{aligned}
& {[H, H]=0, \quad[H, \tilde{H}]=\tilde{H},} \\
& {[\tilde{H}, \tilde{H}]=0 .}
\end{aligned}
$$

Proof. It is straightforward to check that (2.1.2a)-(2.1.2c) are compatible with the graded antisymmetry and Jacobi relations of $D_{1}$. By stability and grading reasons, one has $\left[\mathrm{H}^{i}, \mathrm{H}^{j}\right] \subseteq \mathrm{H}^{i+j}$, where $\mathbf{H}^{0}=\mathbb{R} H, \mathrm{H}^{1}=\mathbb{R} \tilde{H}$ and $\mathbf{H}^{i}=0$ else. So, (2.1.2a) holds. Further, by stability (cfr. eq. (1.2.2)),

$$
[H, \tilde{H}]=a \tilde{H},
$$

where $a \in \mathbb{R}$. Owing to (2.1.1), $a \neq 0$. Renormalize $H$ into $a^{-1} H$. Then, one can set $a=1$ in (2.1.3), getting (2.1.2b). Applying $\partial$ to (2.1.2b), one obtains (2.1.2c). 
Proposition 2.1.2. Let $I: \mathfrak{g} \mapsto \mathrm{D}_{1}$ be a homomorphism such that $I(\mathfrak{g}) \subseteq \mathrm{D}_{1}^{-1}$. Assume that the set $\{I, \tilde{I}\}$ is stable (cfr. def. 1.2.1). For $\xi \in \mathfrak{g}$, let $\mathrm{I}(\xi)$ the linear span of the set $\{I(\xi), \tilde{I}(\xi)\}$. Assume that

$$
\left[\mathrm{I}^{-1}(\xi), \mathrm{I}^{0}(\eta)\right]=\mathrm{I}^{-1}([\xi, \eta]), \quad \xi, \eta \in \mathfrak{g} .
$$

Then, perhaps after substituting I by $\lambda I$ for some suitable $\lambda \in \mathbb{R}_{\times}$, one has

$$
\begin{array}{ll}
{[I(\xi), I(\eta)]} & =0, \quad[I(\xi), \tilde{I}(\eta)]=I([\xi, \eta]), \\
{[\tilde{I}(\xi), \tilde{I}(\eta)]} & =\tilde{I}([\xi, \eta]),
\end{array}
$$

Proof. It is straightforward to check that $(2.1 .5 \mathrm{a})-(2.1 .5 \mathrm{c})$ are compatible with the graded antisymmetry and Jacobi relations of $\mathrm{D}_{1}$. By stability and grading reasons, one has $\left[I^{i}(\xi), I^{j}(\eta)\right] \subseteq I^{i+j}([\xi, \eta])$, where $\mathbf{I}^{-1}(\xi)=\mathbb{R} I(\xi), I^{0}(\xi)=\mathbb{R} \tilde{I}(\xi)$ and $I^{i}(\xi)=0$ else. So, (2.1.5a) holds. Further, by stability (cfr. eq. (1.2.2)),

$$
[I(\xi), \tilde{I}(\eta)]=a I([\xi, \eta])
$$

where $a \in \mathbb{R}$. Owing to (2.1.4), $a \neq 0$, if $[\mathfrak{g}, \mathfrak{g}] \neq 0$, or one can choose $a \neq 0$, if $[\mathfrak{g}, \mathfrak{g}]=0$. Renormalize $I$ into $a^{-1} I$. Then, one can set $a=1$ in (2.1.6), getting (2.1.5b). Applying $\partial$ to this latter relation, one obtains $(2.1 .5 \mathrm{c})$.

Proposition 2.1.3. Assume that $[\mathfrak{g}, \mathfrak{g}] \neq 0$. Assume that $H$ and I satisfy the hypotheses of props. 2.1.1 and 2.1.2, respectively. Assume further that $\{H, \tilde{H}\}$ acts stably on $\{I, \tilde{I}\}$ (cfr. def. 1.2.4). Assume finally that

$$
\left[\mathrm{H}^{0}, \mathrm{I}^{-1}(\xi)\right]=\mathrm{I}^{-1}(\xi), \quad \xi \in \mathfrak{g} .
$$

Then, one has

$$
\begin{aligned}
{[H, I(\xi)] } & =-I(\xi), \quad[H, \tilde{I}(\xi)]=0, \\
{[\tilde{H}, I(\xi)] } & =-\tilde{I}(\xi), \quad[\tilde{H}, \tilde{I}(\xi)]=0, \quad \xi \in \mathfrak{g} .
\end{aligned}
$$

Proof. It is straightforward to check that (2.1.8a)-(2.1.8d) are compatible with the graded antisymmetry and Jacobi relations of $\mathrm{D}_{1}$ and with relations (2.1.2a)-(2.1.2c), (2.1.5a)-(2.1.5c). By stability and grading reasons, one has $\left[\mathrm{H}^{i}, \mathrm{I}^{j}(\xi)\right] \subseteq \mathrm{I}^{i+j}(\xi)$, where 
$\mathrm{H}^{i}, \mathrm{I}^{i}(\xi)$ have been defined above (2.1.3) and (2.1.6), respectively. Taking stability into account (cfr. eq. (1.2.9)), one has

$$
[H, I(\xi)]=a I(\xi), \quad[H, \tilde{I}(\xi)]=b \tilde{I}(\xi),
$$

where $a, b \in \mathbb{R}$. By applying $\partial$ to (2.1.9) and using (2.1.10), one gets

$$
[\tilde{H}, I(\xi)]=(a-b) \tilde{I}(\xi)
$$

Owing to (2.1.7), $a \neq 0$. From (2.1.5b) and the Jacobi identity, one has $[H, I([\xi, \eta])]-$ $[[H, I(\xi)], \tilde{I}(\eta)]-[I(\xi),[H, \tilde{I}(\eta)]]=0$, which, upon using (2.1.9), (2.1.10), gives the relation $-b I([\xi, \eta])=0$. As $[\mathfrak{g}, \mathfrak{g}] \neq 0$ and $I(\zeta) \neq 0$ for $\zeta \neq 0$ by stability, $b=0$. From (2.1.3b) and the Jacobi identity, $[\tilde{H}, I(\xi)]-[H,[\tilde{H}, I(\xi)]]+[[H, I(\xi)], \tilde{H}]=0$, which, upon using (2.1.9)-(2.1.11), gives $a(a+1) I(\xi)=0 . \quad$ As $\mathfrak{g} \neq 0$ and $I(\zeta) \neq 0$ for $\zeta \neq 0, a=-1$. Substituting the found values of $a$ and $b$ into (2.1.9)-(2.1.11), one gets (2.1.8a)-(2.1.8c). (2.1.8d) is obtained by applying $\partial$ to $(2.1 .8 \mathrm{c})$.

For an arbitrary ungraded Lie algebra $\mathfrak{g}$, relations (2.1.2a)-(2.1.2c), (2.1.5a)-(2.1.5c), (2.1.8a)-(2.1.8d) define a $\mathbb{Z}$ graded real Lie algebra $t_{\theta}$. As shown by the above three propositions, when $\mathfrak{g}$ is not Abelian, $\mathrm{t}_{\theta}$ is the unique solution of a suitable set of stability and non degeneracy conditions. When $\mathfrak{g}$ is Abelian, $\mathrm{t}_{\theta}$ is a particular case of a certain class of $\mathbb{Z}$ graded Lie algebras. We shall not investigate this issue any further.

Note that $\mathrm{t}_{\theta} \simeq \mathrm{t}_{0}=: \mathrm{t}$. The $\mathbb{Z}$ graded real Lie algebra $\mathrm{t}$ is called fundamental $N=1$ superstructure of $\mathfrak{g}$. It is defined in terms of the four generators $h, \tilde{h}, i(\xi), \tilde{\imath}(\xi), \xi \in \mathfrak{g}$, satisfying relations (2.1.2a)-(2.1.2c), (2.1.5a)-(2.1.5c), (2.1.8a)-(2.1.8d). More customarily, one sets

$$
\begin{gathered}
k=h, \quad d=-\tilde{h}, \\
j(\xi)=i(\xi), \quad l(\xi)=\tilde{\imath}(\xi), \quad \xi \in \mathfrak{g} .
\end{gathered}
$$

From (2.1.2a)-(2.1.2c), (2.1.5a)-(2.1.5c) and (2.1.8a)-(2.1.8d), one sees that $k, d, j$ and $l$ satisfy the relations

$$
\begin{array}{r}
{[k, k]=0,} \\
{[k, d]=d,} \\
{[k, j(\xi)]=-j(\xi), \quad[k, l(\xi)]=0, \quad \xi \in \mathfrak{g},} \\
{[d, d]=0,}
\end{array}
$$




$$
\begin{gathered}
{[d, j(\xi)]=l(\xi), \quad[d, l(\xi)]=0, \quad \xi \in \mathfrak{g},} \\
{[j(\xi), j(\eta)]=0, \quad[j(\xi), l(\eta)]=j([\xi, \eta]),} \\
{[l(\xi), l(\eta)]=l([\xi, \eta]),}
\end{gathered}
$$

Note that, by (2.1.14), $k$ generates an ungraded Lie subalgebra

$$
\mathrm{i} \simeq \mathbb{R}
$$

of t. i is called the internal symmetry algebra of the fundamental $N=1$ superstructure t.

\subsection{The fundamental $N=2$ superstructure}

Proposition 2.2.1. Let $H_{A} \in \mathrm{D}_{2}^{-1}, A=1,2$. Assume that the set $\left\{H_{A} \mid A=1,2\right\} \cup$ $\left\{H_{A, B} \mid A, B=1,2\right\} \cup\left\{\tilde{H}_{A} \mid A=1,2\right\}$ is stable (cfr. def. 1.2.2). Let $\mathrm{H}$ the linear span of this set. Assume that

$$
\left[\mathrm{H}^{-1}, \mathrm{H}^{0}\right]=\mathrm{H}^{-1} .
$$

Then, perhaps after substituting $H_{A}$ by $\kappa_{A}^{B} H_{B}$ for a suitable matrix $\kappa \in \mathrm{GL}(2, \mathbb{R})$, one has

$$
\begin{array}{ll}
{\left[H_{A}, H_{B}\right]=0,} & {\left[H_{A}, H_{B, C}\right]=\epsilon_{A B} H_{C}} \\
{\left[H_{A}, \tilde{H}_{B}\right]=-H_{A, B},} & {\left[H_{A, C}, H_{B, D}\right]=\epsilon_{A B} H_{C, D}-\epsilon_{D C} H_{B, A}, \quad(2.2 .2 a)-(2.2 .2 f)} \\
{\left[H_{A, C}, \tilde{H}_{B}\right]=-\epsilon_{B C} \tilde{H}_{A},} & {\left[\tilde{H}_{A}, \tilde{H}_{B}\right]=0 .}
\end{array}
$$

Proof. It is straightforward to check that (2.2.2a)-(2.2.2f) are compatible with the graded antisymmetry and Jacobi relations of $\mathrm{D}_{2}$. By stability and grading reasons, one has $\left[\mathrm{H}^{i}, \mathrm{H}^{j}\right] \subseteq \mathrm{H}^{i+j}$, where $\mathrm{H}^{-1}=\bigoplus_{A=1,2} \mathbb{R} H_{A}, \mathrm{H}^{0}=\bigoplus_{A, B=1,2} \mathbb{R} H_{A, B}, \mathrm{H}^{1}=\bigoplus_{A=1,2} \mathbb{R} \tilde{H}_{A}$ and $\mathrm{H}^{i}=0$ else. So, (2.2.2a) holds. Further, by stability (cfr. eq. (1.2.2)),

$$
\left[H_{A}, H_{B, C}\right]=a_{A B C}{ }^{K} H_{K}, \quad\left[H_{A}, \tilde{H}_{B}\right]=b_{A B}{ }^{K L} H_{K, L},
$$

where $a_{A B C}{ }^{D}, b_{A B}{ }^{C D} \in \mathbb{R}$ are certain constants. Applying $\partial_{C}$ to (2.2.2a), one gets $\left[H_{A, C}, H_{B}\right]+\left[H_{B, C}, H_{A}\right]=0$. Substituting (2.2.3) into this relation and exploiting the linear independence of the $H_{A}$, one has $a_{A B C}{ }^{D}+a_{B A C}{ }^{D}=0$, which entails $a_{A B C}{ }^{D}=\epsilon_{A B} a_{C}{ }^{D}$ 
for certain constants $a_{A}^{B} \in \mathbb{R}$. Now, on account of (2.2.1) and (2.2.3), the matrix $a=\left(a_{A}^{B}\right)$ must be invertible. Redefine $H_{A}$ into $(\operatorname{det} a)^{-1} a_{A}{ }^{K} H_{K}$. The new $H_{A}$ satisfy obviously the same assumptions as the old ones. Further, the new $H_{A}$ satisfy relations (2.2.3), where, now, by construction, $a_{A B C}{ }^{D}=\epsilon_{A B} \delta_{C}{ }^{D}$. In this way, we get (2.2.2b). Next, applying $\partial_{D}$ to $(2.2 .2 \mathrm{~b})$, one gets $\left[H_{A, C}, H_{B, D}\right]+\epsilon_{C D}\left[\tilde{H}_{A}, H_{B}\right]=\epsilon_{A B} H_{C, D}$. From this relation, using $(2.2 .2 \mathrm{a}),(2.2 .2 \mathrm{~b})$ and the Jacobi identity, one obtains $\left[H_{A, C},\left[H_{B, D}, H_{E}\right]\right]-$ $\left[H_{B, D},\left[H_{A, C}, H_{E}\right]\right]-\epsilon_{C D}\left[\left[\tilde{H}_{A}, H_{E}\right], H_{B}\right]-\epsilon_{A B}\left[H_{C, D}, H_{E}\right]=0$. Substituting $(2.2 .2 \mathrm{~b})$ and (2.2.4) into this identity and exploiting the linear independence of the $H_{A}$, one finds that $b_{A B}{ }^{C D}=-\delta_{A}{ }^{C} \delta_{B}{ }^{D}$. Substituting this expression back into (2.2.4), one obtains (2.2.2c). Next, substituting (2.2.2c) into the relation $\left[H_{A, C}, H_{B, D}\right]=\epsilon_{A B} H_{C, D}+\epsilon_{D C}\left[\tilde{H}_{A}, H_{B}\right]$ obtained earlier, one gets $(2.2 .2 \mathrm{~d})$. (2.2.2e) follows from applying $\partial_{C}$ to $(2.2 .2 \mathrm{c})$. Finally, (2.2.2f) follows from applying $\partial_{D}$ to $(2.2 .2 \mathrm{e})$.

Proposition 2.2.2. Let $I: \mathfrak{g} \mapsto \mathrm{D}_{2}$ be a linear map such that $I(\mathfrak{g}) \subseteq \mathrm{D}_{2}^{-2}$. Assume that the set $\{I, \tilde{I}\} \cup\left\{I_{, A} \mid A=1,2\right\}$ is stable (cfr. subsect. 1.3). For $\xi \in \mathfrak{g}$, let $\mathrm{I}(\xi)$ be the linear span of $\{I(\xi), \tilde{I}(\xi)\} \cup\left\{I_{, A}(\xi) \mid A=1,2\right\}$. Assume that

$$
\left[\mathrm{I}^{-2}(\xi), \mathrm{I}^{0}(\eta)\right]=\mathrm{I}^{-2}([\xi, \eta]), \quad \xi, \eta \in \mathfrak{g} .
$$

Then, perhaps after substituting I by $\lambda I$ for a suitable $\lambda \in \mathbb{R}_{\times}$, one has

$$
\begin{array}{lll}
{[I(\xi), I(\eta)]=0,} & {\left[I(\xi), I_{, A}(\eta)\right]=0,} \\
{[I(\xi), \tilde{I}(\eta)]=I([\xi, \eta]),} & {\left[I_{, A}(\xi), I_{, B}(\eta)\right]=\epsilon_{A B} I([\xi, \eta]),} & (2.2 .6 a)-(2.2 .6 f) \\
{\left[I_{, A}(\xi), \tilde{I}(\eta)\right]=I_{, A}([\xi, \eta]),} & {[\tilde{I}(\xi), \tilde{I}(\eta)]=\tilde{I}([\xi, \eta]), \quad \xi, \eta \in \mathfrak{g} .}
\end{array}
$$

Proof. It is straightforward to check that (2.2.6a)-(2.2.6f) are compatible with the graded antisymmetry and Jacobi relations of $D_{2}$. By stability and grading reasons, one has $\left[\mathrm{I}^{i}(\xi), \mathrm{I}^{j}(\eta)\right] \subseteq \mathrm{I}^{i+j}([\xi, \eta])$, where $\mathrm{I}^{-2}(\xi)=\mathbb{R} I(\xi), \mathrm{I}^{-1}(\xi)=\bigoplus_{A=1,2} \mathbb{R} I_{, A}(\xi), \mathrm{I}^{0}(\xi)=\mathbb{R} \tilde{I}(\xi)$ and $\mathrm{I}^{i}(\xi)=0$ else. So, (2.2.6a), (2.2.6b) hold. Further, by stability (cfr. eq (1.2.2)), one has

$$
[I(\xi), \tilde{I}(\eta)]=a I([\xi, \eta]),
$$

where $a \in \mathbb{R}$. Owing to $(2.2 .5), a \neq 0$, if $[\mathfrak{g}, \mathfrak{g}] \neq 0$, or one can choose $a \neq 0$, if $[\mathfrak{g}, \mathfrak{g}]=0$. Renormalize, $I$ into $a^{-1} I$. Then, one can set $a=1$ in (2.2.7), getting (2.2.6c). Applying $\partial_{B}$ to $(2.2 .6 \mathrm{~b})$ and using $(2.2 .6 \mathrm{c})$, one gets $(2.2 .6 \mathrm{~d})$ readily. Applying successively $\partial_{A}$ and $\partial_{A} \partial_{B}$ to $(2.2 .6 \mathrm{c})$, one gets $(2.2 .6 \mathrm{e}),(2.2 .6 \mathrm{f})$. 
Proposition 2.2.3. Assume that $[\mathfrak{g}, \mathfrak{g}] \neq 0$. Assume that $H$ and I satisfy the hypotheses of props. 2.2.1 and 2.2.2, respectively. Assume further that $\left\{H_{A} \mid A=1,2\right\} \cup$ $\left\{H_{A, B} \mid A, B=1,2\right\} \cup\left\{\tilde{H}_{A} \mid A=1,2\right\}$ acts stably on $\{I, \tilde{I}\} \cup\left\{I_{, A} \mid A=1,2\right\}$ (cfr. def. 1.2.4). Assume finally that

$$
\left[H^{0}, I^{-2}(\xi)\right]=I^{-2}(\xi), \quad \xi \in \mathfrak{g} .
$$

Then, one has

$$
\begin{aligned}
& {\left[H_{A}, I(\xi)\right]=0, \quad\left[H_{A}, I_{, B}(\xi)\right]=\epsilon_{A B} I(\xi),} \\
& {\left[H_{A}, \tilde{I}(\xi)\right]=0, \quad\left[H_{A, B}, I(\xi)\right]=\epsilon_{A B} I(\xi),} \\
& {\left[H_{A, C}, I_{, B}(\xi)\right]=\epsilon_{A B} I_{, C}(\xi), \quad\left[H_{A, B}, \tilde{I}(\xi)\right]=0,} \\
& {\left[\tilde{H}_{A}, I(\xi)\right]=-I_{, A}(\xi), \quad\left[\tilde{H}_{A}, I_{, B}(\xi)\right]=\epsilon_{A B} \tilde{I}(\xi),} \\
& {\left[\tilde{H}_{A}, \tilde{I}(\xi)\right]=0 . \quad \xi \in \mathfrak{g} .}
\end{aligned}
$$

Proof. It is straightforward to check that (2.2.9a)-(2.2.9i) are compatible with the graded antisymmetry and Jacobi relations of $\mathrm{D}_{2}$ and with (2.2.2a)-(2.2.2f), (2.2.6a)(2.2.6f). By stability and grading reasons, $\left[\mathrm{H}^{i}, \mathrm{I}^{j}(\xi)\right] \subseteq \mathrm{I}^{i+j}(\xi)$, where $\mathrm{H}^{i}$ and $\mathrm{I}^{i}(\xi)$ have been defined above (2.2.3) and (2.2.7), respectively. So, (2.2.9a) holds. Further, by stability (cfr. eq. (1.2.9)),

$$
\left[H_{A}, I_{, B}(\xi)\right]=a_{A}{ }^{K} \epsilon_{K B} I(\xi) \quad\left[H_{A}, \tilde{I}(\xi)\right]=b_{A}{ }^{K} I_{, K}(\xi),
$$

where $a_{A}{ }^{B}, b_{A}{ }^{B} \in \mathbb{R}$. Applying $\partial_{C}$ to (2.2.9a) and (2.2.10), and using (2.2.10), (2.2.11) to cast the relations so obtained, one gets further

$$
\left[H_{A, B}, I(\xi)\right]=a_{A}{ }^{K} \epsilon_{K B} I(\xi), \quad\left[H_{A, C}, I_{, B}(\xi)\right]=a_{A}{ }^{K} \epsilon_{K B} I_{, C}(\xi)+\epsilon_{B C} b_{A}{ }^{K} I_{, K}(\xi) .
$$

Now, from (2.2.6e) and the Jacobi identity, $\left[\left[H_{A}, I_{, B}(\xi)\right], \tilde{I}(\eta)\right]-\left[I_{, B}(\xi),\left[H_{A}, \tilde{I}(\eta)\right]\right]-$ $\left[H_{A}, I_{, B}([\xi, \eta])\right]=0$. Substituting (2.2.10), (2.2.11) into this relation and using (2.2.6e), one obtains $b_{A}{ }^{K} \epsilon_{K B} I([\xi, \eta])=0$. As $[\mathfrak{g}, \mathfrak{g}] \neq 0$ and $I(\zeta) \neq 0$ for $\zeta \neq 0$ by stability, one has $b_{A}{ }^{B}=0$. Next, from (2.2.2b) and the Jacobi identity, one has $\left[H_{A, C},\left[H_{D}, I_{, B}(\xi)\right]\right]-$ $\left[H_{D},\left[H_{A, C}, I_{, B}(\xi)\right]\right]-\epsilon_{A D}\left[H_{C}, I_{, B}(\xi)\right]=0$. Substituting (2.2.10), (2.2.12), (2.2.13) into this relation, one gets $-\epsilon_{A D} \epsilon^{B K}\left[-a_{C}{ }^{L} a_{L}{ }^{K}+a_{L}{ }^{L} a_{C}{ }^{K}-a_{C}{ }^{K}\right] I(\xi)=0$. Since $\mathfrak{g} \neq 0$ and $I(\zeta) \neq 0$ for $\zeta \neq 0,-a_{A}{ }^{K} a_{K}{ }^{B}+a_{K}{ }^{K} a_{A}{ }^{B}-a_{A}{ }^{B}=0$. It is a simple algebraic exercise to 
show that there are only two 2 by 2 real matrices satisfying this relation: $a_{A}{ }^{B}=0$ and $a_{A}{ }^{B}=\delta_{A}^{B}$. The first solution is not admissible, because, by (2.2.12), it would violate (2.2.8). So, the second holds. By substituting the values of $a_{A}{ }^{B}$ and $b_{A}{ }^{B}$ thus found in $(2.2 .10)-(2.2 .13)$, one gets $(2.2 .9 \mathrm{~b})-(2.2 .9 \mathrm{e})$. (2.2.9f) follows from applying $\partial_{B}$ to $(2.2 .9 \mathrm{c})$. $(2.2 .9 \mathrm{~g})$ follows from applying $\partial_{C}$ to $(2.2 .9 \mathrm{~d})$ and using $(2.2 .9 \mathrm{e})$ to express the result. Applying successively $\partial_{B}$ and $\partial_{B} \partial_{C}$ to $(2.2 .9 \mathrm{~g})$, one gets $(2.2 .9 \mathrm{~h}),(2.2 .9 \mathrm{i})$.

For an arbitrary ungraded Lie algebra $\mathfrak{g}$, relations $(2.2 .2 \mathrm{a})-(2.2 .2 \mathrm{f}),(2.2 .6 \mathrm{a})-(2.2 .6 \mathrm{f})$, (2.2.9a)-(2.2.9i) define a $\mathbb{Z}$ graded real Lie algebra $t_{\theta}$. As shown by the above three propositions, when $\mathfrak{g}$ is not Abelian, $\mathrm{t}_{\theta}$ is the unique solution of a suitable set of stability and non degeneracy conditions. When $\mathfrak{g}$ is Abelian, $\mathrm{t}_{\theta}$ is a particular case of a certain class of $\mathbb{Z}$ graded Lie algebras. We shall not investigate this matter any further. Note the analogy to the $N=1$ case.

Note that $\mathrm{t}_{\theta} \simeq \mathrm{t}_{0}=\mathrm{t}$. The $\mathbb{Z}$ graded real Lie algebra $\mathrm{t}$ is called fundamental $N=2$ superstructure of $\mathfrak{g}$. It is defined in terms of the six generators $h_{A}, h_{A, B}, \tilde{h}_{A}, i(\xi), i_{, A}(\xi)$, $\tilde{\imath}(\xi), \xi \in \mathfrak{g}$ satisfying relations $(2.2 .2 \mathrm{a})-(2.2 .2 \mathrm{f}),(2.2 .6 \mathrm{a})-(2.2 .6 \mathrm{f}),(2.2 .9 \mathrm{a})-(2.2 .9 \mathrm{i})$. To make contact with ref. [31], one sets

$$
\begin{array}{cl}
t_{A B}=h_{A, B}+h_{B, A}, \quad k=\epsilon^{K L} h_{K, L}, & (2.2 .14 a)-(2.2 .14 d) \\
u_{A}=h_{A}, & d_{A}=-\tilde{h}_{A}, \\
j(\xi)=i(\xi), \quad j_{A}(\xi)=i_{, A}(\xi), \quad l(\xi)=\tilde{\imath}(\xi), \quad \xi \in \mathfrak{g} . & (2.2 .15 a)-(2.2 .15 c)
\end{array}
$$

From $(2.2 .2 \mathrm{a})-(2.2 .2 \mathrm{f}),(2.2 .6 \mathrm{a})-(2.2 .6 \mathrm{f})$ and $(2.2 .9 \mathrm{a})-(2.2 .9 \mathrm{i})$, one sees that $t_{A B}, k, u_{A}$, $d_{A}, j, j_{A}$ and $l$ satisfy the relations

$$
\begin{array}{cl}
{\left[t_{A C}, t_{B D}\right]=\epsilon_{A B} t_{C D}+\epsilon_{C B} t_{A D}+\epsilon_{A D} t_{B C}+\epsilon_{C D} t_{B A},} \\
{\left[k, t_{A B}\right]=0, \quad[k, k]=0,} \\
{\left[t_{A C}, u_{B}\right]=\epsilon_{A B} u_{C}+\epsilon_{C B} u_{A},} & {\left[k, u_{A}\right]=-u_{A},} \\
{\left[t_{A C}, d_{B}\right]=\epsilon_{A B} d_{C}+\epsilon_{C B} d_{A},} & {\left[k, d_{A}\right]=d_{A},} \\
{\left[t_{A B}, j(\xi)\right]=0,} & {[k, j(\xi)]=-2 j(\xi),} \\
{\left[t_{A C}, j_{B}(\xi)\right]=\epsilon_{A B} j_{C}(\xi)+\epsilon_{C B} j_{A}(\xi),} & {\left[k, j_{A}(\xi)\right]=-j_{A}(\xi),} \\
{\left[t_{A B}, l(\xi)\right]=0,} & {[k, l(\xi)]=0, \quad \xi \in \mathfrak{g},}
\end{array}
$$




$$
\begin{gathered}
{\left[u_{A}, u_{B}\right]=0, \quad\left[d_{A}, u_{B}\right]=\frac{1}{2}\left(t_{A B}+\epsilon_{A B} k\right),} \\
{\left[d_{A}, d_{B}\right]=0,} \\
{\left[u_{A}, j(\xi)\right]=0, \quad\left[u_{A}, j_{B}(\xi)\right]=\epsilon_{A B} j(\xi),} \\
{\left[u_{A}, l(\xi)\right]=0,} \\
{\left[d_{A}, j_{B}(\xi)\right]=-\epsilon_{A B} l(\xi), \quad\left[d_{A}, l(\xi)\right]=j_{A}(\xi),} \\
{[j(\xi), j(\eta)]=0, \quad \xi \in \mathfrak{g},} \\
{[j(\xi), l(\eta)]=j([\xi, \eta]), \quad\left[j(\xi), j_{A}(\eta)\right]=0,} \\
{\left[j_{A}(\xi), l(\eta)\right]=j_{A}([\xi, \eta]), \quad[l(\xi), l(\eta)]=l([\xi, \eta]), \quad \xi, \eta \in \mathfrak{g} .}
\end{gathered}
$$

Note that, from (2.2.16a)-(2.2.16c), $t_{A B}, k$ generate an ungraded Lie subalgebra

$$
\mathrm{i} \simeq \mathfrak{s l}(2, \mathbb{R}) \oplus \mathbb{R}
$$

of $\mathrm{t}$. i is called the internal symmetry algebra of the $N=2$ fundamental superstructure $\mathrm{t}$ and plays an important role.

\section{The Weil algebra}

Let $d$ be a $\mathbb{Z}$ graded real Lie algebra. Let $z$ be a $\mathbb{Z}$ graded real left $d$ module algebra with unity 1 , where the action of $d$ on $z$ is derivative, i. e. it obeys the graded Leibniz rule. Finally, let $\mathfrak{g}$ be an ungraded real Lie algebra.

\subsection{The $N=1$ case}

Proposition 3.1.1. Assume that $[\mathfrak{g},[\mathfrak{g}, \mathfrak{g}]] \neq 0$. Assume that $H$ and $I$ satisfy the hypotheses of props. 2.1.1 and 2.1.2, respectively. Let $W: \mathfrak{g}^{\vee} \mapsto \mathrm{Z}_{1}$ be a homomorphism such that $W\left(\mathfrak{g}^{\vee}\right) \subseteq \mathrm{Z}_{1}^{1}$. Suppose $\{W, \tilde{W}\}$ is stable and that $\{H, \tilde{H}\}$ and $\{I, \tilde{I}\}$ act stably and 1-stably on $\{W, \tilde{W}\}$ (cfr. defs. 1.2.3, 1.2.4), respectively, the module action of $\mathfrak{g}$ on $\mathfrak{g}^{\vee}$ being the coadjoint one. For $\mu \in \mathfrak{g}^{\vee}$, denote by $\mathrm{W}(\mu)$ the linear span of $\{W(\mu), \tilde{W}(\mu)\}$. Assume that

$$
\mathrm{I}^{-1}(\xi) \mathrm{W}^{1}(\mu)=\mathbb{R} 1
$$


for some $\xi \in \mathfrak{g}$, for fixed $\mu \in \mathfrak{g}^{\vee}, \mu \neq 0$, and for some $\mu \in \mathfrak{g}^{\vee}$, for fixed $\xi \in \mathfrak{g}, \xi \neq 0$. Finally, suppose that

$$
\mathbf{I}^{0}(\xi) \mathbf{W}^{1}(\mu)=\mathbf{W}^{1}\left(\operatorname{ad}^{\vee} \xi \mu\right)
$$

for $\xi \in \mathfrak{g}$ and $\mu \in \mathfrak{g}^{\vee}$. Then, after perhaps redefining $W$ into $W \circ f$ for some invertible linear map $f: \mathfrak{g}^{\vee} \mapsto \mathfrak{g}^{\vee}$ and viewing $W$ as an element of $\mathbf{Z}_{1} \otimes \mathfrak{g}$, one has

$$
\begin{aligned}
& H W=W, \quad H \tilde{W}=2 \tilde{W}, \\
& \tilde{H} W=-\tilde{W}, \quad \tilde{H} \tilde{W}=0,
\end{aligned}
$$

$$
\begin{array}{ll}
I(\xi) W=\xi, & I(\xi) \tilde{W}=-[\xi, W], \\
\tilde{I}(\xi) W=-[\xi, W], \quad \tilde{I}(\xi) \tilde{W}=-[\xi, \tilde{W}], \quad \xi \in \mathfrak{g} .
\end{array}
$$

Proof. It is straightforward to check that (3.1.3a)-(3.1.3d) and (3.1.4a)-(3.1.4d) are compatible with relations (2.1.2a)-(2.1.2c), (2.1.5a)-(2.1.5c) and (2.1.8a)-(2.1.8d). By stability, 1-stability and grading reasons, $\mathrm{H}^{i} \mathrm{~W}^{j}(\mu) \subseteq \mathrm{W}^{i+j}(\mu)$ and $\mathrm{I}^{i}(\xi) \mathrm{W}^{j}(\mu) \subseteq$ $\mathrm{W}^{i+j}\left(\operatorname{ad}^{\vee} \xi \mu\right) \oplus \mathbb{R} \delta_{i+j, 0} 1$, where $\mathrm{H}^{i}$ and $\mathrm{I}^{i}(\xi)$ are given above (2.1.3) and (2.1.6), respectively, and $\mathbf{W}^{1}(\mu)=\mathbb{R} W(\mu), \mathbf{W}^{2}(\mu)=\mathbb{R} \tilde{W}(\mu)$ and $\mathbf{W}^{i}(\mu)=0$, else. We note further that $H 1=0$ and $I(\xi) 1=0$ as $H$ and $I(\xi)$ act as derivations and $1^{2}=1$. By stability (cfr. eq. $(1.2 .9))$, one has thus

$$
H W(\mu)=a W(\mu), \quad \tilde{H} W(\mu)=b \tilde{W}(\mu)
$$

where $a, b \in \mathbb{R}$. Further, by 1 -stability (cfr. eq. (1.2.9)), one has

$$
I(\xi) W(\mu)=\varphi(\xi, \mu), \quad \tilde{I}(\xi) W(\mu)=c W\left(\operatorname{ad}^{\vee} \xi \mu\right),
$$

where $\varphi: \mathfrak{g} \times \mathfrak{g}^{\vee} \mapsto \mathbb{R}$ is a bilinear map and $c \in \mathbb{R}$. Next, from (2.1.8a), one has the relation $([H, I(\xi)]+I(\xi)) W(\mu)=0$, which, upon using (3.1.5), (3.1.7), gives (1 a) $\varphi(\xi, \mu)=0$. As $\varphi \neq 0$, by (3.1.1), (3.1.7), $a=1$. Next, by (3.1.2), (3.1.8), $c \neq 0$. From (2.1.5c), one has the relation $([\tilde{I}(\xi), \tilde{I}(\eta)]-\tilde{I}([\xi, \eta])) W(\mu)=0$, which, using (3.1.8), yields $c(c+1) W\left(\operatorname{ad}^{\vee}[\xi, \eta] \mu\right)=0$. As, $c \neq 0$ and $[\mathfrak{g},[\mathfrak{g}, \mathfrak{g}]] \neq 0$ and $W(\nu) \neq 0$ for $\nu \neq 0$ by stability, one has $c=-1$. Next, we write $\varphi(\xi, \mu)=\langle\mu, \phi(\xi)\rangle$, where $\phi: \mathfrak{g} \mapsto \mathfrak{g}$ is a linear map, which on account of (3.1.1), (3.1.7) is invertible. From (2.1.5b), one has the relation $([I(\xi), \tilde{I}(\eta)]-I([\xi, \eta])) W(\mu)=0$, which, using (3.1.7), (3.1.8), gives the relation 
$\langle\mu,[\phi(\xi), \eta]-\phi([\xi, \eta])\rangle=0$. So, $[\phi, \operatorname{ad} \eta]=0$. Redefine $W$ into $W \circ \phi^{\vee-1}$. After doing so, (3.1.7) holds with $\varphi(\xi, \mu)=\langle\mu, \xi\rangle$, while (3.1.8) is formally invariant. In this way, one obtains (3.1.4a), (3.1.4c). (3.1.4b) follows from applying $\partial$ to (3.1.4a) and using (3.1.4c). (3.1.4d) follows from applying $\partial$ to (3.1.4c). Next, by $(2.1 .8 \mathrm{c})$, one has the relation $[[\tilde{H}, I(\xi)]+\tilde{I}(\xi)] W(\mu)=0$, which, by using (3.1.6), (3.1.4a) $-(3.1 .4 \mathrm{c})$ gives $-(b+$ 1) $W\left(\operatorname{ad}^{\vee} \xi \mu\right)=0$. As $[\mathfrak{g}, \mathfrak{g}] \neq 0$ and $W(\nu) \neq 0$ for $\nu \neq 0, b=-1$. By substituting the values of $a$ and $b$ thus found into (3.1.5), (3.1.6), one gets (3.1.3a). (3.1.3c). (3.1.3b) follows from applying $\partial$ to $(3.1 .3 \mathrm{a})$ and using (3.1.3c). (3.1.3d) follows from applying $\partial$ to $(3.1 .3 \mathrm{c})$.

For an arbitrary ungraded Lie algebra $\mathfrak{g}$, relations $(3.1 .3 \mathrm{a})-(3.1 .3 \mathrm{~d}),(3.1 .4 \mathrm{a})-(3.1 .4 \mathrm{~d})$ define a $\mathbb{Z}$ graded real left module algebra $w_{\theta}$ of the graded real Lie algebra $t_{\theta}$ (cfr. subsect. 2.1). As shown by the above propositions, when $[\mathfrak{g},[\mathfrak{g}, \mathfrak{g}]] \neq 0, w_{\theta}$ is the unique solution of a suitable set of stability and non degeneracy conditions. Else, $w_{\theta}$ is a particular case of a certain class of $\mathbb{Z}$ graded left module algebra of $t_{\theta}$.

Note that $\mathrm{w}_{\theta} \simeq \mathrm{w}_{0}=: \mathrm{w}$. $\mathrm{w}$ is called $N=1$ Weil algebra of $\mathfrak{g}$ and is a $\mathbb{Z}$ graded real left module algebra of the fundamental $N=1$ superstructure $\mathrm{t}$ of $\mathfrak{g}$ (cfr. subsect. $2.1)$. It is defined in terms of the generators $1, w, \tilde{w}$ and the derivations $h, \tilde{h}, i(\xi), \tilde{\imath}(\xi)$, $\xi \in \mathfrak{g}$, satisfying relations $(3.1 .3 \mathrm{a})-(3.1 .3 \mathrm{~d}),(3.1 .4 \mathrm{a})-(3.1 .4 \mathrm{~d})$. However, in the standard treatment, $w$ is usually presented as follows. Define

$$
\omega=w, \quad \phi=\tilde{w}+(1 / 2)[w, w]
$$

Then, one has

$$
\begin{gathered}
k \omega=\omega, \quad k \phi=2 \phi, \\
d \omega=\phi-(1 / 2)[\omega, \omega], \quad d \phi=-[\omega, \phi], \\
j(\xi) \omega=\xi, \quad j(\xi) \phi=0, \\
l(\xi) \omega=-[\xi, \omega], \quad l(\xi) \phi=-[\xi, \phi], \quad \xi \in \mathfrak{g},
\end{gathered}
$$

where $k, d, j, l$ are given by $(2.1 .12 \mathrm{a}),(2.1 .12 \mathrm{~b}),(2.1 .13 \mathrm{a}),(2.1 .13 \mathrm{~b})$. Note that $\omega$ is just another name for $w$. $\phi$ is by construction 'horizontal', i. e. satisfying (3.1.12b).

3.2. The $N=2$ case 
Proposition 3.2.1. Assume that $[\mathfrak{g},[\mathfrak{g}, \mathfrak{g}]] \neq 0$. Assume that $H_{A}, A=1,2$, and $I$ satisfy the hypotheses of props. 2.2.1 and 2.2.2, respectively. Let $W_{A}: \mathfrak{g}^{\vee} \mapsto \mathrm{Z}_{2}, A=1,2$, be homomorphisms such that $W\left(\mathfrak{g}^{\vee}\right) \subseteq \mathrm{Z}_{2}^{1}$. Suppose that $\left\{W_{A} \mid A=1,2\right\} \cup\left\{W_{A, B} \mid A, B=\right.$ $1,2\} \cup\left\{\tilde{W}_{A} \mid A=1,2\right\}$ is stable and that $\left\{H_{A} \mid A=1,2\right\} \cup\left\{H_{A, B} \mid A, B=1,2\right\} \cup\left\{\tilde{H}_{A} \mid A=1,2\right\}$ and $\{I, \tilde{I}\} \cup\left\{I_{, A} \mid A=1,2\right\}$ act stably and 1-stably on $\left\{W_{A} \mid A=1,2\right\} \cup\left\{W_{A, B} \mid A, B=\right.$ $1,2\} \cup\left\{\tilde{W}_{A} \mid A=1,2\right\}$ (cfr. defs. 1.2.3, 1.2.4), respectively, the module action of $\mathfrak{g}$ on $\mathfrak{g}^{\vee}$ being the coadjoint one. For $\mu \in \mathfrak{g}^{\vee}$, denote by $\mathrm{W}(\mu)$ the linear span of $\left\{W_{A}(\mu) \mid A=\right.$ $1,2\} \cup\left\{W_{A, B}(\mu) \mid A, B=1,2\right\} \cup\left\{\tilde{W}_{A}(\mu) \mid A=1,2\right\}$. Assume that

$$
\mathrm{I}^{-1}(\xi) \mathrm{W}^{1}(\mu)=\mathbb{R} 1,
$$

for some $\xi \in \mathfrak{g}$, for fixed $\mu \in \mathfrak{g}^{\vee}, \mu \neq 0$, and for some $\mu \in \mathfrak{g}^{\vee}$, for fixed $\xi \in \mathfrak{g}, \xi \neq 0$. Finally, suppose that

$$
\mathbf{I}^{0}(\xi) \mathbf{W}^{1}(\mu)=\mathbf{W}^{1}\left(\operatorname{ad}^{\vee} \xi \mu\right),
$$

for $\xi \in \mathfrak{g}$ and $\mu \in \mathfrak{g}^{\vee}$. Then, after perhaps redefining $W_{A}$ into $\lambda_{A}{ }^{B} W_{B} \circ f$ for some matrix $\lambda \in \mathrm{GL}(2, \mathbb{R})$ and invertible linear map $f: \mathfrak{g}^{\vee} \mapsto \mathfrak{g}^{\vee}$ and viewing $W$ as an element of $\mathrm{Z}_{2} \otimes \mathfrak{g}$, one has

$$
\begin{array}{ll}
H_{A} W_{B}=0, & H_{A} W_{B, C}=-\epsilon_{B C} W_{A}, \\
H_{A} \tilde{W}_{B}=-W_{A, B}-W_{B, A}, & H_{A, C} W_{B}=-\epsilon_{B C} W_{A}, \\
H_{A, C} W_{B, D}=\epsilon_{C B} W_{A, D}-\epsilon_{D C} W_{B, A}, & H_{A, C} \tilde{W}_{B}=-\epsilon_{B C} \tilde{W}_{A}-\epsilon_{A C} \tilde{W}_{B}, \\
\tilde{H}_{A} W_{B}=-W_{B, A}, & \tilde{H}_{A} W_{B, C}=\epsilon_{A C} \tilde{W}_{B}, \\
\tilde{H}_{A} \tilde{W}_{B}=0, &
\end{array}
$$

$$
\begin{array}{ll}
I(\xi) W_{A}=0, & I(\xi) W_{A, B}=\epsilon_{A B} \xi, \\
I(\xi) \tilde{W}_{A}=-\left[\xi, W_{A}\right], & I_{, A}(\xi) W_{B}=\epsilon_{A B} \xi, \\
I_{, A}(\xi) W_{B, C}=-\epsilon_{A C}\left[\xi, W_{B}\right], & I_{, A}(\xi) \tilde{W}_{B}=-\left[\xi, W_{B, A}\right], \\
\tilde{I}(\xi) W_{A}=-\left[\xi, W_{A}\right], & \tilde{I}(\xi) W_{A, B}=-\left[\xi, W_{A, B}\right], \\
\tilde{I}(\xi) \tilde{W}_{A}=-\left[\xi, \tilde{W}_{A}\right], & \xi \in \mathfrak{g} .
\end{array}
$$


Proof. It is straightforward to check that (3.2.3a)-(3.2.3i) and (3.2.4a)-(3.2.4i) are compatible with relations $(2.2 .2 \mathrm{a})-(2.2 .2 \mathrm{f}),(2.2 .6 \mathrm{a})-(2.2 .6 \mathrm{f})$ and $(2.2 .9 \mathrm{a})-(2.2 .9 \mathrm{i})$. By stability, 1-stability and grading reasons, one has $\mathrm{H}^{i} \mathrm{~W}^{j}(\mu) \subseteq \mathrm{W}^{i+j}(\mu)$ and $\mathrm{I}^{i}(\xi) \mathrm{W}^{j}(\mu) \subseteq$ $\mathrm{W}^{i+j}\left(\mathrm{ad}^{\vee} \xi \mu\right) \oplus \mathbb{R} \delta_{i+j, 0} 1$, where $\mathrm{H}^{i}$ and $\mathrm{I}^{i}(\xi)$ are given above (2.2.3) and (2.2.7), respectively, and $\mathrm{W}^{1}(\mu)=\bigoplus_{A=1,2} \mathbb{R} W_{A}(\mu), \mathrm{W}^{2}(\mu)=\bigoplus_{A, B=1,2} \mathbb{R} W_{A, B}(\mu), \mathrm{W}^{3}(\mu)=\bigoplus_{A=1,2} \mathbb{R} \tilde{W}_{A}(\mu)$ and $\mathrm{W}^{i}(\mu)=0$, else. We note further that $H_{A} 1=0$ and $I(\xi) 1=0$ as $H_{A}$ and $I(\xi)$ act as derivations and $1^{2}=1$. Thus, by stability, (3.2.3a) holds and one has

$$
H_{A, C} W_{B}(\mu)=a_{A B C}{ }^{K} W_{K}(\mu), \quad \tilde{H}_{A} W_{B}(\mu)=b_{A B}{ }^{K L} W_{K, L}(\mu)
$$

where $a_{A B C}{ }^{D}, b_{A B}{ }^{C D} \in \mathbb{R}$. Further, by 1-stability, (3.2.4a) holds and one has

$$
I_{, A}(\xi) W_{B}(\mu)=\varphi_{A B}(\xi, \mu), \quad \tilde{I}(\xi) W_{A}(\mu)=c_{A}{ }^{K} W_{K}\left(\operatorname{ad}^{\vee} \xi \mu\right),
$$

where $\varphi_{A B}: \mathfrak{g} \times \mathfrak{g}^{\vee} \mapsto \mathbb{R}$ is a bilinear map and $c_{A}{ }^{B} \in \mathbb{R}$. Now, by $(2.2 .2 \mathrm{~d}),\left(\left[H_{A, C}, H_{B, D}\right]-\right.$ $\left.\epsilon_{A B} H_{C, D}+\epsilon_{D C} H_{B, A}\right) W_{E}(\mu)=0$. Upon substituting (3.2.5) into this relation, one obtains $\left(a_{A E C}{ }^{K} a_{B K D}{ }^{L}-a_{B E D}{ }^{K} a_{A K C}{ }^{L}+\epsilon_{A B} a_{C E D}{ }^{L}-\epsilon_{D C} a_{B E A}{ }^{L}\right) W_{L}(\mu)=0$. Since the $W_{A}(\nu)$ are linearly independent for $\nu \neq 0, a_{A E C}{ }^{K} a_{B K D}{ }^{F}-a_{B E D}{ }^{K} a_{A K C}{ }^{F}+\epsilon_{A B} a_{C E D}{ }^{F}-$ $\epsilon_{D C} a_{B E A}{ }^{F}=0$. As is easy to see, this relation implies that the matrices $a_{A B}=\left(a_{A C B}{ }^{D}\right)$ form a 2 dimensional representation of the Lie algebra $\mathfrak{s l}(2, \mathbb{R}) \oplus \mathbb{R}$. A simple Lie algebraic analysis shows that either $i) a_{A B C}{ }^{D}=-\epsilon_{B C} \delta_{A}{ }^{D}-\epsilon_{A C} a \delta_{B}{ }^{D}$ or $\left.i i\right) a_{A B C}{ }^{D}=$ $-\epsilon_{A C} a_{B}{ }^{D}$ up to equivalence, where $a, a_{A}{ }^{B} \in \mathbb{R}$. From $(2.2 .9 \mathrm{e})$, one has the relation $\left(\left[H_{A, C}, I_{, B}(\xi)\right]-\epsilon_{A B} I_{, C}(\xi)\right) W_{D}(\mu)=0$, which upon using (3.2.5), (3.2.7), entails that $-a_{A D C}{ }^{K} \varphi_{B K}(\xi, \mu)-\epsilon_{A B} \varphi_{C D}(\xi, \mu)=0$. Substituting the expression of $a_{A C B}{ }^{D}$ found above into this identity, one gets after some simple rearrangements $a \varphi_{A B}(\xi, \mu)=0$ in case $i$ and $\varphi_{A B}(\xi, \mu)=0$ in case $i i$. As $\varphi_{A B}$ cannot vanish by (3.2.1), (3.2.7), only case $i$ with $a=0$ can occur. So, $a_{A B C}{ }^{D}=-\epsilon_{B C} \delta_{A}{ }^{D}$. Using this expression, one sees then that $\epsilon_{C D} \varphi_{A B}(\xi, \mu)-\epsilon_{A B} \varphi_{C D}(\xi, \mu)=0$, which implies that $\varphi_{A B}(\xi, \mu)=\epsilon_{A B} \varphi(\xi, \mu)$ for some bilinear map $\varphi: \mathfrak{g} \times \mathfrak{g}^{\vee} \mapsto \mathbb{R}$. Next, by (3.2.2), (3.2.8), the matrix $c=\left(c_{A}{ }^{B}\right)$ is invertible. From $(2.2 .6 \mathrm{f})$, one has the relation $[[\tilde{I}(\xi), \tilde{I}(\eta)]-\tilde{I}([\xi, \eta])] W_{A}(\mu)=0$, which, using (3.2.8), yields $c_{A}^{K}\left(c_{K}{ }^{L}+\delta_{K}{ }^{L}\right) W_{L}\left(\operatorname{ad}^{\vee}[\xi, \eta] \mu\right)=0$. As, $c$ is invertible, $[\mathfrak{g},[\mathfrak{g}, \mathfrak{g}]] \neq 0$ and the $W_{A}(\nu)$ are linearly independent for $\nu \neq 0$, one has $c_{A}{ }^{B}=-\delta_{A}{ }^{B}$. Next, we write $\varphi(\xi, \mu)=\langle\mu, \phi(\xi)\rangle$, where $\phi: \mathfrak{g} \mapsto \mathfrak{g}$ is a linear map, which on account of (3.2.1) is invertible. From $(2.2 .6 \mathrm{e})$, one has the relation $\left(\left[I_{, A}(\xi), \tilde{I}(\eta)\right]-I_{, A}([\xi, \eta])\right) W_{B}(\mu)=0$, which, using (3.2.7), (3.2.8), gives the relation $\epsilon_{A B}\langle\mu,[\phi(\xi), \eta]-\phi([\xi, \eta])\rangle=0$. So, $[\phi$, ad $\eta]=0$. Redefine $W$ into $W \circ \phi^{\vee-1}$. After doing so, (3.2.7) holds with $\varphi(\xi, \mu)=\langle\mu, \xi\rangle$, while (3.2.8) is formally invariant. In this way, one obtains $(3.2 .4 \mathrm{~d}),(3.2 .4 \mathrm{~g})$. By applying $\partial_{B}$ to $(3.2 .4 \mathrm{a})$ and 
using $(3.2 .4 \mathrm{~d})$, one obtains (3.2.4b). Applying $\partial_{C}$ to $(3.2 .4 \mathrm{~d})$ and using $(3.2 .4 \mathrm{~g})$, one obtains (3.2.4e). Applying $\partial_{C}$ to $(3.2 .4 \mathrm{~b})$ and using (3.2.4e), one gets (3.2.4c). Applying $\partial_{B}$, $\partial_{C} \partial_{B}$ to $(3.2 .4 \mathrm{~g})$, one gets $(3.2 .4 \mathrm{~h}),(3.2 .4 \mathrm{i})$, respectively. Applying $\partial_{D}$ to $(3.2 .4 \mathrm{e})$ and using $(3.2 .4 \mathrm{~h})$, one gets $(3.2 .4 \mathrm{f})$. Next, by $(2.2 .9 \mathrm{~h}),\left(\left[\tilde{H}_{A}, I_{, B}(\xi)\right]-\epsilon_{A B} \tilde{I}(\xi)\right) W_{C}(\mu)=0$. Using (3.2.6), (3.2.4d), (3.2.4e), (3.2.4g), this yields $\left(\epsilon_{A B} \delta_{C}{ }^{K}+b_{A C}{ }^{K L} \epsilon_{L B}\right) W_{K}\left(\mathrm{ad}^{\vee} \xi \mu\right)=0$. As $[\mathfrak{g}, \mathfrak{g}] \neq 0$ and the $W_{A}(\nu)$ are linearly independent for $\nu \neq 0$, one has $b_{A B}{ }^{C D}=-\delta_{A}{ }^{D} \delta_{B}{ }^{C}$. Substituting the expressions of $a_{A B C}{ }^{D}$ and $b_{A B}{ }^{C D}$ obtained into (3.2.5), (3.2.6), one obtains $(3.2 .3 \mathrm{~d}),(3.2 .3 \mathrm{~g})$. By applying $\partial_{B}$ to $(3.2 .3 \mathrm{a})$ and using $(3.2 .3 \mathrm{~d})$, one obtains $(3.2 .4 \mathrm{~b})$. Applying $\partial_{D}$ to $(3.2 .3 \mathrm{~d})$ and using $(3.2 .3 \mathrm{~g})$, one obtains (3.2.3e). Applying $\partial_{D}$ to $(3.2 .3 \mathrm{~b})$ and using $(3.2 .3 \mathrm{e})$, one gets $(3.2 .3 \mathrm{c})$. Applying $\partial_{C}, \partial_{D} \partial_{C}$ to $(3.2 .3 \mathrm{~g})$, one gets $(3.2 .3 \mathrm{~h})$, (3.2.3i), respectively. Applying $\partial_{E}$ to $(3.2 .3 \mathrm{e})$ and using $(3.2 .3 \mathrm{~h})$, one gets $(3.2 .3 \mathrm{f})$.

For an arbitrary ungraded Lie algebra $\mathfrak{g}$, relations (3.2.3a)-(3.2.3i), (3.2.4a)-(3.2.4i) define a $\mathbb{Z}$ graded real left module algebra $w_{\theta}$ of the graded real Lie algebra $t_{\theta}$ (cfr. subsect. 2.2 ). As shown by the above proposition, when $[\mathfrak{g},[\mathfrak{g}, \mathfrak{g}]] \neq 0, \mathrm{w}_{\theta}$ is the unique solution of a suitable set of stability and non degeneracy conditions. Else, $w_{\theta}$ is a particular case of a certain class of $\mathbb{Z}$ graded left module algebra of $\mathrm{t}_{\theta}$. Note the analogy to the $N=1$ case

Note that $\mathrm{w}_{\theta} \simeq \mathrm{w}_{0}=: \mathrm{w} . \mathrm{w}$ is called $N=2$ Weil algebra of $\mathfrak{g}$ and is a $\mathbb{Z}$ graded real left module algebra of the fundamental $N=2$ superstructure $\mathrm{t}$ of $\mathfrak{g}$ (cfr. subsect. 2.2). It is defined in terms of the generators $1, w_{A}, w_{A, B}, \tilde{w}_{A}$ of the derivations $h_{A}, h_{A, B}, \tilde{h}_{A}, i(\xi)$, $i_{, A}(\xi), \tilde{\imath}(\xi), \xi \in \mathfrak{g}$ satisfying relations $(3.2 .3 \mathrm{a})-(3.2 .3 \mathrm{i}),(3.2 .4 \mathrm{a})-(3.2 .4 \mathrm{i})$. To make contact with ref. [31], we shall present $w$ as follows. Define

$$
\begin{array}{ll}
\omega_{A}=w_{A}, & \phi_{A B}=\frac{1}{2}\left(w_{A, B}+w_{B, A}+\left[w_{A}, w_{B}\right]\right), \\
\gamma=-\frac{1}{2} \epsilon^{K L} w_{K, L}, & \rho_{A}=-\frac{1}{2} \tilde{w}_{A}-\frac{1}{2} \epsilon^{K L}\left[w_{K}, w_{A, L}\right]-\frac{1}{6} \epsilon^{K L}\left[w_{K},\left[w_{L}, w_{A}\right]\right] .
\end{array}
$$

Then, one has

$$
\begin{array}{ll}
t_{A C} \omega_{B}=\epsilon_{A B} \omega_{C}+\epsilon_{C B} \omega_{A}, & k \omega_{A}=\omega_{A}, \\
t_{A C} \phi_{B D}=\epsilon_{A B} \phi_{C D}+\epsilon_{C B} \phi_{A D}+\epsilon_{A D} \phi_{B C}+\epsilon_{C D} \phi_{B A}, & k \phi_{A B}=2 \phi_{A B}, \\
t_{A B} \gamma=0, & k \gamma=2 \gamma, \\
t_{A C} \rho_{B}=\epsilon_{A B} \rho_{C}+\epsilon_{C B} \rho_{A}, & k \rho_{A}=3 \rho_{A},
\end{array}
$$




$$
\begin{array}{ll}
u_{A} \omega_{B}=0, & u_{A} \phi_{B C}=0 \\
u_{A} \gamma=-\omega_{A}, & u_{A} \rho_{B}=\phi_{A B} \\
d_{A} \omega_{B}=-\frac{1}{2}\left[\omega_{A}, \omega_{B}\right]+\phi_{A B}-\epsilon_{A B} \gamma, & d_{A} \phi_{B C}=-\left[\omega_{A}, \phi_{B C}\right]+\epsilon_{A B} \rho_{C}+\epsilon_{A C} \rho_{B}, \\
d_{A} \gamma=-\frac{1}{2}\left[\omega_{A}, \gamma\right]+\rho_{A} & d_{A} \rho_{B}=-\left[\omega_{A}, \rho_{B}\right]-\frac{1}{2} \epsilon^{K L}\left[\phi_{K A}, \phi_{L B}\right] \\
+\frac{1}{2} \epsilon^{K L}\left[\omega_{K}, \phi_{L A}-\frac{1}{6}\left[\omega_{L}, \omega_{A}\right]\right], & j(\xi) \phi_{A B}=0, \\
j(\xi) \omega_{A}=0, & j(\xi) \rho_{A}=0, \\
j(\xi) \gamma=\xi, & j_{A}(\xi) \phi_{B C}=0, \\
j(\xi) \omega_{B}=\epsilon_{A B} \xi, & j(\xi) \rho_{B}=0, \\
j(\xi) \gamma=-\frac{1}{2}\left[\xi, \omega_{A}\right], & l(\xi) \phi_{A B}=-\left[\xi, \phi_{A B}\right], \\
l(\xi) \omega_{A}=-\left[\xi, \omega_{A}\right], & l(\xi) \rho_{A}=-\left[\xi, \rho_{A}\right], \\
l(\xi) \gamma=-[\xi, \gamma], & \xi \in \mathfrak{g},
\end{array}
$$

where $t_{A, B}, k, u_{A}, d_{A}, j, j_{A}, l$ are given by by $(2.2 .14 \mathrm{a})-(2.2 .14 \mathrm{~d}),(2.2 .15 \mathrm{a})-(2.2 .15 \mathrm{c})$. Note that $\omega_{A}$ is just another name for $w_{A}$. $\gamma$ contains the information about $\tilde{h}_{A} w_{B}$ not exhausted by $\phi_{A B}$. By construction $\phi_{A B}$ and $\rho_{A}$ are 'horizontal', i. e. satisfy (3.2.12b), $(3.2 .12 \mathrm{~d}),(3.2 .12 \mathrm{f}),(3.2 .12 \mathrm{~h})$.

\section{Superoperations and their cohomologies}

Let $\mathfrak{g}$ be an ungraded real Lie algebra.

4.1. $N=1$ superoperations and their cohomologies

Definition 4.1.1. a is called an $N=1 \mathfrak{g}$ superoperation if:

i) a is a $\mathbb{Z}$ graded real left module algebra of the fundamental $N=1$ superstructure $\mathrm{t}$ of $\mathfrak{g}$ (cfr. subsect. 2.1);

ii) the action of $\mathrm{t}$ on a is derivative;

iii) a is completely reducible under the internal symmetry algebra i of $\mathrm{t}$ (cfr. subsect. 2.1), the spectrum of the invariant $k$ of $\mathrm{i}$ is integer and the eigenspace $\mathrm{a}^{p}$ of $k$ of the eigenvalue $p \in \mathbb{Z}$ is precisely the degree $p$ subspace of a. 
So, a is acted upon by four graded derivations $h, \tilde{h}, i(\xi), \tilde{\imath}(\xi), \xi \in \mathfrak{g}$, of degree 0 , $+1,-1,0$, respectively, satisfying relations $(2.1 .2 \mathrm{a})-(2.1 .2 \mathrm{c}),(2.1 .5 \mathrm{a})-(2.1 .5 \mathrm{c}),(2.1 .8 \mathrm{a})-$ (2.1.8d), or, equivalently, by four graded derivations $k, d, j(\xi), l(\xi), \xi \in \mathfrak{g}$, of degree $0,+1$, -1 , 0, respectively, satisfying relations $(2.1 .14),(2.1 .15 \mathrm{a})-(2.1 .15 \mathrm{c}),(2.1 .16),(2.1 .17 \mathrm{a})-$ (2.1.17b), (2.1.18a)-(2.1.18c), the two sets of derivations being related as in (2.1.12a), (2.1.12b), (2.1.13a), (2.1.13b).

Proposition 4.1.1. If $\mathrm{a}^{(r)}, r=1,2$, are two $N=1 \mathfrak{g}$ superoperations, then their graded tensor product $\mathrm{a}=\mathrm{a}^{(1)} \hat{\otimes} \mathrm{a}^{(2)}$ is also an $N=1 \mathfrak{g}$ superoperation.

Proof. Indeed a satisfies the conditions stated in def. 4.1.1.

Let a be an $N=1 \mathfrak{g}$ superoperation.

The pair $(\mathrm{a}, d)$ is an ordinary differential complex, as the graded derivation $d$ has degree +1 and $[d, d]=0$. Its cohomology $H^{*}(\mathrm{a})$, defined in the usual way by

$$
H^{p}(\mathrm{a})=\left(\operatorname{ker} d \cap \mathrm{a}^{p}\right) / d \mathrm{a}^{p-1}, \quad p \in \mathbb{Z},
$$

is the ordinary cohomology of the superoperation. Define

$$
\mathrm{a}_{\text {basic }}=\bigcap_{\xi \in \mathfrak{g}} \operatorname{ker} j(\xi) \cap \operatorname{ker} l(\xi)
$$

By (2.1.17a), (2.1.17b), a $_{\text {basic }}$ is $d$ invariant. So, $\left(\mathrm{a}_{\text {basic }}, d\right)$ is also a differential complex. Its cohomology $H_{\text {basic }}^{*}(\mathrm{a})$

$$
H_{\text {basic }}^{p}(\mathrm{a})=\left(\operatorname{ker} d \cap \mathrm{a}_{\text {basic }}^{p}\right) / d \mathrm{a}_{\text {basic }}^{p-1}, \quad p \in \mathbb{Z},
$$

is the basic cohomology of the superoperation.

Proposition 4.1.2. Each non zero (basic) cohomology class of degree p defines a 1 dimensional representation of the internal Lie algebra i of invariant $p$.

Proof. Set $k[x]=[k x]=p[x]$ for $[x] \in H^{p}(\mathrm{a})\left([x] \in H_{\text {basic }}^{p}(\mathrm{a})\right)$ with arbitrary representative $x \in \mathrm{a}^{n, p}\left(x \in \mathrm{a}_{\text {basic }}^{n, p}\right)$.

Though the above proposition is trivial, it is nevertheless interesting because of its non trivial generalization to higher $N$.

\section{2. $N=2$ superoperations and their cohomologies}


Definition 4.2.1. a is called an $N=2 \mathfrak{g}$ superoperation if:

i) a is a $\mathbb{Z}$ graded real left module algebra of the fundamental $N=2$ superstructure $\mathrm{t}$ of $\mathfrak{g}$ (cfr. subsect. 2.2);

ii) the action of $\mathrm{t}$ on a is derivative;

iii) a is completely reducible under the internal symmetry algebra i of $\mathrm{t}$ (cfr. subsect. 2.2), the spectrum of the invariant $k$ of $\mathrm{i}$ is integer and the eigenspace $\mathrm{a}^{p}$ of $k$ of the eigenvalue $p \in \mathbb{Z}$ is precisely the degree $p$ subspace of a.

So, a is acted upon by six graded derivations $h_{A}, h_{A, B}, \tilde{h}_{A}, i(\xi), i_{, A}(\xi), \tilde{\imath}(\xi), \xi \in \mathfrak{g}$, of degree $-1,0,+1,-2,-1,0$, respectively, satisfying relations $(2.2 .2 \mathrm{a})-(2.2 .2 \mathrm{f})$, (2.2.6a)(2.2.6f), (2.2.9a)-(2.2.9i), or, equivalently, by seven graded derivations $t_{A B}, k, u_{A}, d_{A}$, $j(\xi), j_{A}(\xi), l(\xi), \xi \in \mathfrak{g}$, of degree $0,0,-1,+1,-2,-1,0$, respectively, satisfying relations $(2.2 .16 \mathrm{a})-(2.2 .16 \mathrm{c}),(2.2 .17 \mathrm{a})-(2.2 .17 \mathrm{j}),(2.2 .18 \mathrm{a})-(2.2 .18 \mathrm{c}),(2.2 .19 \mathrm{a})-(2.2 .19 \mathrm{f}),(2.2 .20 \mathrm{a})-$ $(2.2 .20 \mathrm{f})$, the two sets of derivations being related as in $(2.2 .14 \mathrm{a})-(2.2 .14 \mathrm{~d}),(2.2 .15 \mathrm{a})-$ $(2.2 .15 \mathrm{c})$.

Besides $k$, i possesses another invariant, namely

$$
c=-\frac{1}{8} \epsilon^{K L} \epsilon^{M N} t_{K M} t_{L N}
$$

An irreducible representation of $\mathrm{i}$ is completely characterized up to equivalence by the values of $c$ and $k$, which we parametrize as $\frac{1}{4}\left(n^{2}-1\right)$ and $p$, respectively, where $n \in \mathbb{N}$ and $p \in \mathbb{Z}$. $n$ is nothing but the dimension of the representation. Being completely reducible under i, a organizes into irreducible representations of $i$. We denote by $a^{n, p}$ the eigenspace of $c, k$ of eigenvalues $\frac{1}{4}\left(n^{2}-1\right), p$, respectively. It follows that a has a finer grading than the original one.

Proposition 4.2.1. If $\mathrm{a}^{(r)}, r=1,2$, are two $N=2 \mathfrak{g}$ superoperations, then their graded tensor product $\mathrm{a}=\mathrm{a}^{(1)} \hat{\otimes} \mathrm{a}^{(2)}$ is also an $N=2 \mathfrak{g}$ superoperation.

Proof. Indeed a satisfies the conditions stated in def. 4.2.1.

Let a be an $N=2 \mathfrak{g}$ superoperation.

The graded derivations $d_{A}$ have degree +1 and satisfy $\left[d_{A}, d_{B}\right]=0$. So, one may define a double differential complex $\left(\mathrm{a}, d_{A}\right)$. We do not define cohomology in the usual way, as the standard definition would not be covariant with respect to i. Instead, we propose the following definition generalizing that of ref. [31]. The ordinary cohomology $H^{*}(\mathrm{a})$ is labelled by the values of the invariants $c, k$ of $\mathrm{i}$ and is defined as

$$
H^{n, p}(\mathrm{a})=\left(\cap_{A=1,2} \operatorname{ker} d_{A} \cap \mathrm{a}^{n, p}\right) / \frac{1}{2} \epsilon^{K L} d_{K} d_{L} \mathrm{a}^{n, p-2}, \quad(n, p) \in \mathbb{N} \times \mathbb{Z} .
$$


The basic subspace of a is defined as

$$
\mathrm{a}_{\mathrm{basic}}=\bigcap_{\xi \in \mathfrak{g}} \operatorname{ker} j(\xi) \cap \cap_{A=1,2} \operatorname{ker} j_{A}(\xi) \cap \operatorname{ker} l(\xi) .
$$

Using (2.2.18d)-(2.2.18f), one can show that $\mathrm{a}_{\text {basic }}$ is $d_{A}$ invariant. So, $\left(\mathrm{a}_{\text {basic }}, d_{A}\right)$ is also a double differential complex. Its cohomology $H_{\text {basic }}^{*}(a)$ is defined

$$
H_{\text {basic }}^{n, p}(\mathrm{a})=\left(\cap_{A=1,2} \operatorname{ker} d_{A} \cap \mathrm{a}_{\text {basic }}^{n, p}\right) / \frac{1}{2} \epsilon^{K L} d_{K} d_{L} \mathrm{a}_{\text {basic }}^{n, p-2}, \quad(n, p) \in \mathbb{N} \times \mathbb{Z},
$$

where $\mathrm{a}_{\text {basic }}^{n, p}=\mathrm{a}^{n, p} \cap \mathrm{a}_{\mathrm{basic}}$, and is the basic cohomology of the superoperation.

The (basic) cohomology of any $N=2$ superoperation a is structurally restricted, as indicated by the following.

Proposition 4.2.2. One has

$$
H^{n, p}(\mathrm{a})=0, \quad \text { for } p \neq \pm n+1 \text {. }
$$

Similarly,

$$
H_{\text {basic }}^{n, p}(\mathrm{a})=0, \quad \text { for } p \neq \pm n+1 .
$$

Proof. It is convenient for the time being to revert to the original basis $h_{A}, h_{B, C}$, $\tilde{h}_{D}$ of $\mathrm{t}$, which allows for a more compact notation. Let $x \in$ a such that $\tilde{h}_{A} x=0$. Using $(2.2 .2 \mathrm{~b}),(2.2 .2 \mathrm{c})$, it is easy to show that

$$
\left[h_{A}+\epsilon^{K L} h_{K} h_{L, A}\right] x-\tilde{h}_{A} \frac{1}{2} \epsilon^{K L} h_{K} h_{L} x=0 .
$$

Apply now $\tilde{h}_{B}$ to the left hand side of this equation and contract with $\epsilon^{B A}$. After a short calculation exploiting $(2.2 .2 \mathrm{c}),(2.2 .2 \mathrm{e})$, one gets

$$
\left[-\frac{1}{2} \epsilon^{K L} \epsilon^{M N} h_{K, M} h_{L, N}+\frac{1}{2} \epsilon^{K L} h_{K, L}\right] x-\frac{1}{2} \epsilon^{K L} \tilde{h}_{K} \tilde{h}_{L} \frac{1}{2} \epsilon^{M N} h_{M} h_{N} x=0 .
$$

Using the relation $h_{A, B}=\frac{1}{2}\left(t_{A B}-\epsilon_{A B} k\right)$, following from (2.2.14a), (2.2.14b), and (2.2.14c), $(2.2 .14 \mathrm{~d})$ and $(4.2 .1)$ in (4.2.8), one gets finally

$$
\left[c+\frac{1}{4}\left(1-(k-1)^{2}\right)\right] x-\frac{1}{2} \epsilon^{K L} d_{K} d_{L} \frac{1}{2} \epsilon^{M N} u_{M} u_{N} x=0 .
$$

If $x \in \mathrm{a}^{n, p},(4.2 .9)$ yields

$$
\frac{1}{4}\left[n^{2}-(p-1)^{2}\right] x-\frac{1}{2} \epsilon^{K L} d_{K} d_{L} \frac{1}{2} \epsilon^{M N} u_{M} u_{N} x=0
$$

(4.2.10) yields (4.2.5) immediately. (4.2.6) follows also from (4.2.10) upon checking that for $x \in \mathrm{a}_{\text {basic }}, \frac{1}{2} \epsilon^{M N} h_{M} h_{N} x \in \mathrm{a}_{\text {basic }}$ as well, by (2.2.9a)-(2.2.9c). 
Proposition 4.2.3. The non trivial elements of $H^{n, p}(\mathrm{a})\left(H_{\text {basic }}^{n, p}(\mathrm{a})\right)$ fill irreducible representations of the internal symmetry algebra i of invariants $n, p$.

Proof. By (2.2.17c), (2.2.17d), if $x \in \mathrm{a}^{n, p} \cap \cap_{A=1,2} \operatorname{ker} d_{A}$, then $t_{A B} x, k x \in \mathrm{a}^{n, p} \cap$ $\cap_{A=1,2} \operatorname{ker} d_{A}$ as well. Further, if $x \in \frac{1}{2} \epsilon^{K L} d_{K} d_{L} \mathrm{a}^{n, p-2}, t_{A B} x, k x \in \frac{1}{2} \epsilon^{K L} d_{K} d_{L} \mathrm{a}^{n, p-2}$, also. One thus defines $t_{A B}[x]=\left[t_{A B} x\right]$ and $k[x]=[k x]$, for any $[x] \in H^{n, p}(\mathrm{a})$ with arbitrary representative $x \in \mathrm{a}^{n, p} \cap \cap_{A=1,2} \operatorname{ker} d_{A}$. This yields the first part of the proposition. The statement extends to basic cohomology, by noting that $t_{A B} x, k x \in \mathrm{a}_{\text {basic }}^{n, p}$ whenever $x \in$ $\mathrm{a}_{\text {basic }}^{n, p}$, by $(2.2 .17 \mathrm{e})-(2.2 .17 \mathrm{j})$.

Recall that the only irreducible $n$ dimensional module of $\mathrm{i}=\mathfrak{s l}(2, \mathbb{R}) \oplus \mathbb{R}$ is the completely symmetric tensor space $\bigvee^{n-1} \mathbb{R}^{2}$ up to equivalence. Hence, one has a tensor factorization of the form

$$
\begin{gathered}
H^{n, p}(\mathrm{a})=K^{n, p} \otimes \bigvee^{n-1} \mathbb{R}^{2}, \\
H_{\text {basic }}^{n, p}(\mathrm{a})=K_{\text {basic }}^{n, p} \otimes \bigvee^{n-1} \mathbb{R}^{2},
\end{gathered}
$$

for certain real vector spaces $K^{n, p}, K_{\text {basic }}^{n, p}$

\section{The Weil superoperation and its cohomologies}

Let $\mathfrak{g}$ be an ungraded real Lie algebra.

5.1. The $N=1$ case

Let $\mathrm{w}$ be the $N=1$ Weil algebra of $\mathfrak{g}$ (cfr. subsect. 3.1). Then, $\mathrm{w}$ is an $N=1$ $\mathfrak{g}$ superoperation (cfr. def. 4.1.1) called $N=1$ Weil superoperation. Indeed, as shown in subsect. $3.1, \mathrm{w}$ is a $\mathbb{Z}$ graded real left module algebra of the fundamental $N=1$ superstructure $t$ of $\mathfrak{g}$, the action of $t$ on $w$ is derivative and $w$ is obviously completely reducible under the internal symmetry algebra $\mathrm{i}$ with $k$ acting as the degree operator of $\mathrm{w}$ by (3.1.10a), (3.1.10b).

Theorem 5.1.1. $H^{p}(\mathrm{w})=0$ for $p \neq 0$ and

$$
H^{0}(\mathrm{w}) \simeq \mathbb{R}
$$

Similarly, $H_{\text {basic }}^{p}(\mathrm{w})=0$, for $p \neq 2 s$ with $s \geq 0$, and

$$
H_{\text {basic }}^{2 s}(\mathrm{w}) \simeq\left(\bigvee^{s} \mathfrak{g}^{\vee}\right)_{\mathrm{ad}^{\vee} \mathfrak{g}}, \quad s \geq 0
$$

where $\left(\bigvee^{s} \mathfrak{g}^{\vee}\right)_{\mathrm{ad}} \mathfrak{g}_{\mathfrak{g}}$ denotes the subspace of symmetrized tensor product $\bigvee^{s} \mathfrak{g}^{\vee}$ spanned by the elements which are invariant under the coadjoint action of $\mathfrak{g}$. 
Proof. Below, we shall use the following notation. Let $r \in \Lambda^{*} \mathfrak{g}^{\vee} \otimes \bigvee^{*} \mathfrak{g}^{\vee}$. Let $\xi \in \Pi \mathfrak{g}$, $\eta \in \mathfrak{g}$, where $\Pi \mathfrak{g}$ is the Grassmann odd partner of $\mathfrak{g}$. We denote by $r(\xi, \eta)$ the evaluation of $r$ on $\sum_{p, q \geq 0} \xi^{\otimes p} \otimes \eta^{\otimes q}$. Every element $z \in \mathrm{w}$ is of the form $z=r(w, \tilde{w})$ for some $r \in \Lambda^{*} \mathfrak{g}^{\vee} \otimes \bigvee^{*} \mathfrak{g}^{\vee}$ uniquely determined by $z$. As $\operatorname{deg} w=1, \operatorname{deg} \tilde{w}=2, \mathrm{w}^{p}=0$ for $p<0$ and $\mathrm{w}^{0}=\mathbb{R} 1$. Hence, $H^{p}(\mathrm{w})=0$ for $p<0$ and $H^{0}(\mathrm{w}) \simeq \mathbb{R}$, trivially. Let $\mathrm{w}^{p>0}=\bigoplus_{p>0} \mathrm{w}^{p}$. $\mathrm{w}^{p>0}$ is acted upon by the graded derivations $h, \tilde{h}$ and two more graded derivations $i^{*}, \tilde{\imath}^{*}$ of degree $-1,0$, respectively, defined by

$$
\begin{aligned}
& i^{*} w=0, \quad i^{*} \tilde{w}=w, \\
& \tilde{\imath}^{*} w=w, \quad \tilde{\imath}^{*} \tilde{w}=\tilde{w} .
\end{aligned}
$$

Identify $i^{*}, \tilde{\imath}^{*}$ with the linear maps $i^{*}(x)=x i^{*}, \tilde{\imath}^{*}(x)=x \tilde{\imath}^{*}, x \in \mathbb{R}$, Then, $h, \tilde{h}, i^{*}, \tilde{\imath}^{*}$ satisfy relations (2.1.2a)-(2.1.2c), (2.1.5a)-(2.1.5c), (2.1.8a)-(2.1.8d) with $\mathfrak{g}=\mathbb{R}$. It follows that $\mathrm{w}^{p>0}$ is an $N=1 \mathbb{R}$ superoperation. Switch now to the derivations $k, d, j^{*}, l^{*}$ defined by $(2.1 .12 \mathrm{a}),(2.1 .12 \mathrm{~b}),(2.1 .13 \mathrm{a}),(2.1 .13 \mathrm{~b})$. By $(2.1 .17 \mathrm{a}), j^{*}$ is a homotopy operator for $d$, for $l^{*}$ commutes with $j^{*}$ and $d$, by $(2.1 .17 \mathrm{~b}),(2.1 .18 \mathrm{~b})$, and $l^{*}$ is invertible on $\mathrm{w}^{p>0}$, by $(5.1 .3 \mathrm{c}),(5.1 .3 \mathrm{~d})$ and the definition of $\mathrm{w}^{p>0}$. Thus, the cohomology of $d$ is trivial on $\mathrm{w}^{p>0}$. This proves the first part of the theorem. Every element $z \in \mathrm{w}_{\mathrm{basic}}$ is of the form $z=r(\phi)$ for some $r \in\left(\bigvee^{*} \mathfrak{g}^{\vee}\right)_{\mathrm{ad}^{\vee} \mathfrak{g}}$ uniquely determined by $z$. Indeed, $z=r(\omega, \phi)$ for a unique $r \in \Lambda^{*} \mathfrak{g}^{\vee} \otimes \bigvee^{*} \mathfrak{g}^{\vee}$, by an argument similar to that employed earlier, and, by (3.1.12a)-(3.1.12d), the basicity conditions $j(\xi) r(\omega, \phi)=0, l(\xi) r(\omega, \phi)=0$ imply that $r$ has polynomial degree 0 in the first argument and is $\operatorname{ad}^{\vee} \mathfrak{g}$ invariant. It follows that $\mathrm{w}_{\text {basic }}^{p}=0$ for $p \neq 2 s$ with $s \geq 0$, as $\operatorname{deg} \phi=2$. So, $H_{\text {basic }}^{p}(\mathrm{w})=0$ for $p \neq 2 s$ with $s \geq 0$. Let $s \geq 0$. If $z=r(\phi)$ with $r \in\left(\bigvee^{s} \mathfrak{g}^{\vee}\right)_{\mathrm{ad}^{\vee} \mathfrak{g}}$, then $d z=0$, by (3.1.11b) and the ad ${ }^{\vee} \mathfrak{g}$ invariance of $r$. Hence, $\mathrm{w}_{\text {basic }}^{2 s} \cap \operatorname{ker} d=\mathrm{w}_{\text {basic }}^{2 s}$. We thus have a linear injection $\mu: \mathrm{w}_{\text {basic }}^{2 s} \cap \operatorname{ker} d \mapsto\left(\bigvee^{s} \mathfrak{g}^{\vee}\right)_{\mathrm{ad}^{\vee} \mathfrak{g}}$ given by $z \mapsto r$. As, $\mathrm{w}_{\text {basic }}^{2 s-1}=0, \mu$ induces a linear bijection $\hat{\mu}: H_{\text {basic }}^{2 s}(\mathrm{w}) \mapsto\left(\bigvee^{s} \mathfrak{g}^{\vee}\right)_{\mathrm{ad}^{\vee} \mathfrak{g}}$.

5.2. The $N=2$ case

Let $\mathrm{w}$ be the $N=2$ Weil algebra of $\mathfrak{g}$ (cfr. subsect. 3.2). Then, $\mathrm{w}$ is an $N=2$ $\mathfrak{g}$ superoperation (cfr. def. 4.2.1) called $N=2$ Weil superoperation. Indeed, as shown in subsect. $3.2, \mathrm{w}$ is a $\mathbb{Z}$ graded real left module algebra of the fundamental $N=2$ superstructure $t$ of $\mathfrak{g}$, the action of $t$ on $w$ is derivative and $w$ is obviously completely reducible under the internal symmetry algebra $\mathrm{i}$ with $k$ acting as the degree operator of $\mathrm{w}$ by $(3.2 .10 \mathrm{a})-(3.2 .10 \mathrm{~h})$. 
Theorem 5.2.1. $H^{n, p}(\mathrm{w})=0$, for $(n, p) \neq(1,0)$, and

$$
H^{1,0}(\mathrm{w}) \simeq \mathbb{R}
$$

Similarly, $H_{\text {basic }}^{n, p}(\mathrm{w})=0$, for $(n, p) \neq(1,0),(2 s, 2 s+1)$ with $s>0$, and

$$
H_{\text {basic }}^{1,0}(\mathrm{w}) \simeq \mathbb{R}, \quad H_{\text {basic }}^{2 s, 2 s+1}(\mathrm{w}) \simeq\left(\bigvee^{s} \mathfrak{g}^{\vee}\right)_{\mathrm{ad}^{\vee} \mathfrak{g}} \otimes \bigvee^{2 s-1} \mathbb{R}^{2}, \quad s>0
$$

Proof. Below, we shall use the following notation. Let $r \in \Lambda^{*}\left(\mathfrak{g}^{\vee} \otimes \otimes^{a} \mathbb{R}^{2}\right) \otimes \bigvee^{*}\left(\mathfrak{g}^{\vee} \otimes\right.$ $\left.\bigotimes^{b} \mathbb{R}^{2}\right)$. Let $\xi \in \Pi \mathfrak{g} \otimes \bigotimes^{a} \mathbb{R}^{2 \vee}, \eta \in \mathfrak{g} \bigotimes^{b} \mathbb{R}^{2 \vee}$. We denote by $r(\xi, \eta)$ the evaluation of $r$ on $\sum_{p, q \geq 0} \xi^{\otimes p} \otimes \eta^{\otimes q}$. The above notation can be straightforwardly generalized to the case where there are several $\xi$ and $\eta$. Every element $z \in \mathrm{w}$ is of the form $z=r(w, w, \tilde{w})$ for some $r \in \Lambda^{*}\left(\mathfrak{g}^{\vee} \otimes \mathbb{R}^{2}\right) \otimes \bigvee^{*}\left(\mathfrak{g}^{\vee} \otimes \otimes^{2} \mathbb{R}^{2}\right) \otimes \Lambda^{*}\left(\mathfrak{g}^{\vee} \otimes \mathbb{R}^{2}\right)$ uniquely determined by $z$. As $\operatorname{deg} w_{A}=1, \operatorname{deg} w_{A, B}=2, \operatorname{deg} \tilde{w}_{A}=3, \mathrm{w}^{n, p}=0$, for $p<0$, and $\mathrm{w}^{n, 0}=\mathbb{R} \delta_{n, 1} 1$. So, $H^{n, p}(\mathrm{w})=0$, for $p<0$, and $H^{n, 0}(\mathrm{w}) \simeq \delta_{n, 1} \mathbb{R}$, trivially. Let $\mathrm{w}^{p>0}=\bigoplus_{n \in \mathbb{N}, p>0} \mathrm{w}^{n, p} \cdot \mathrm{w}^{p>0}$ is acted upon by the graded derivations $h_{A}, h_{A, B}, \tilde{h}_{A}$ and three more graded derivations $i, i_{, A}, \tilde{\imath}$ of degree $-2,-1,0$, respectively, defined by

$$
\begin{array}{lll}
i^{*} w_{A}=0, & i^{*} w_{A, B}=0, & i^{*} \tilde{w}_{A}=w_{A}, \\
i^{*}{ }_{A} w_{B}=0, & i^{*}{ }_{A} w_{B, C}=-\epsilon_{C A} w_{B}, & i^{*}{ }_{A} \tilde{w}_{B}=w_{B, A}, \\
\tilde{\imath}^{*} w_{A}=w_{A}, & \tilde{\imath}^{*} w_{A, B}=w_{A, B}, & \tilde{\imath}^{*} \tilde{w}_{A}=\tilde{w}_{A} .
\end{array}
$$

Identify $i^{*}, i^{*}{ }_{A}, \tilde{\imath}^{*}$ with the linear maps $i^{*}(x)=x i^{*}, i^{*}{ }_{A}(x)=x i^{*},{ }_{A}, \tilde{\imath}^{*}(x)=x \tilde{\imath}^{*}$,

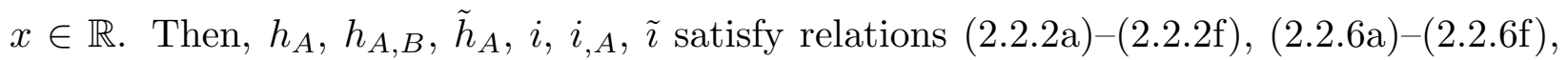
$(2.2 .9 \mathrm{a})-(2.2 .9 \mathrm{i})$ with $\mathfrak{g}=\mathbb{R}$. From this fact, it is easy to see that $\mathrm{w}^{p>0}$ is an $N=2$ $\mathbb{R}$ superoperation. Switch now to the derivations $t_{A, B}, k, u_{A}, d_{A}, j^{*}, j_{A}^{*}, l^{*}$ defined by $(2.2 .14 \mathrm{a})-(2.2 .14 \mathrm{~d}),(2.2 .15 \mathrm{a})-(2.2 .15 \mathrm{c})$. By $(2.2 .19 \mathrm{e}), j^{*}{ }_{A}$ is a homotopy operator for $d_{A}$, for $l^{*}$ commutes with $j^{*} A$ and $d_{A}$, by (2.2.19f), (2.2.20e), and $l^{*}$ is invertible on $w^{p>0}$, by $(5.2 .3 \mathrm{~g})-(5.2 .3 \mathrm{i})$ and the definition of $\mathrm{w}^{p>0}$. Indeed, using (2.2.19e), (2.2.19f), (2.2.20e), one can show that

$$
\left[\frac{1}{2} \epsilon^{K L} d_{K} d_{L}, \frac{1}{2} \epsilon^{M N} j^{*}{ }_{M} j^{*}{ }_{N}\right]=-l^{*}\left(l^{*}+\epsilon^{K L} j^{*}{ }_{K} d_{L}\right)
$$

where, by $(2.2 .17 \mathrm{~g}),(2.2 .17 \mathrm{~h}), \frac{1}{2} \epsilon^{M N} j^{*}{ }_{M} j^{*}{ }_{N}$ maps $\mathrm{w}^{n, p}$ into $\mathrm{w}^{n, p-2}$. Thus, the cohomology of $d_{A}$ is trivial on $\mathrm{w}^{p>0}$. This proves the first part of the theorem. Let us examine next the second part. As $\mathrm{w}^{n, p}=0$, for $p<0$, and $\mathrm{w}^{n, 0}=\mathbb{R} \delta_{n, 1} 1$, as shown earlier, and 1 is obviously 
basic, $\mathrm{w}_{\text {basic }}^{n, p}=0$, for $p<0$, and $\mathrm{w}_{\text {basic }}^{n, 0}=\mathbb{R} \delta_{n, 1} 1$. Consequently, $H_{\text {basic }}^{n, p}(\mathrm{w})=0$ for $p<0$. and $H_{\text {basic }}^{n, 0}(\mathrm{w}) \simeq \delta_{n, 1} \mathbb{R}$. On the other hand, by prop. 4.2.2, eq. (4.2.6), $H_{\text {basic }}^{n, p}(\mathrm{w})=0$ for $p \neq \pm n+1$. So, the only potentially non vanishing cohomology spaces which are left are $H_{\text {basic }}^{n, n+1}(\mathrm{w}), n \geq 1$, which we shall analyze next. Every element $z \in \mathrm{w}_{\text {basic }}$ is of the form $z=$ $r(\phi, \rho)$ for some $r \in\left(\bigvee^{*}\left(\mathfrak{g}^{\vee} \otimes \bigvee^{2} \mathbb{R}^{2}\right) \otimes \Lambda^{*}\left(\mathfrak{g}^{\vee} \otimes \mathbb{R}^{2}\right)\right)_{\text {ad } \vee \mathfrak{g}}$ uniquely determined by $z$. Indeed, $z=r(\omega, \gamma, \phi, \rho)$, for a unique $r \in \Lambda^{*}\left(\mathfrak{g}^{\vee} \otimes \mathbb{R}^{2}\right) \otimes \bigvee^{*} \mathfrak{g}^{\vee} \otimes \bigvee^{*}\left(\mathfrak{g}^{\vee} \otimes \bigvee^{2} \mathbb{R}^{2}\right) \otimes \Lambda^{*}\left(\mathfrak{g}^{\vee} \otimes \mathbb{R}^{2}\right)$, by an argument similar to that employed earlier in the proof, and, by (3.2.12a)-(3.2.12l), the basicity conditions $j(\xi) r(\omega, \gamma, \phi, \rho)=0, j_{A}(\xi) r(\omega, \gamma, \phi, \rho)=0, l(\xi) r(\omega, \gamma, \phi, \rho)=0$ imply that $r$ has polynomial degree 0 in the first two arguments and is ad $\mathfrak{g}$ invariant. Let $z=r(\phi, \rho) \in \mathrm{w}_{\text {basic }}^{n, n+1}$. From (3.2.10c), (3.2.10d), (3.2.10g), (3.2.10h) and the representation theory of $\mathbf{i}=\mathfrak{s l}(2, \mathbb{R}) \oplus \mathbb{R}$, one knows that the total number of internal indices $A=1,2$ and the total degree carried by $\phi_{A B}, \rho_{A}$ in each monomial of $r(\phi, \rho)$ must be $n-1+2 \nu$ and $n+1$, respectively, where $2 \nu$ is the number of indices contracted by means of $\epsilon^{A B}$. Further, the $n-1$ uncontracted indices are totally symmetrized. So, the numbers $m_{\phi}, m_{\rho}$ of occurences of $\phi_{A B}, \rho_{A}$ in a given monomial must satisfy the equations $2 m_{\phi}+1 m_{\rho}=n-1+2 \nu$, $2 m_{\phi}+3 m_{\rho}=n+1$. Taking into account that $m_{\phi}, m_{\rho}$ are non negative integers, one finds that $\nu=0, m_{\phi}=s-1, m_{\rho}=1$, for $n=2 s$ with $s \geq 1$, and $\nu=1, m_{\phi}=s, m_{\rho}=0$, for $n=2 s-1$ with $s \geq 2$. Thus, the most general $z \in \mathrm{w}_{\text {basic }}^{n, n+1}$ is of the form

$$
\begin{aligned}
& z=u^{A_{1} \cdots A_{2 s-1}}\left(\phi_{A_{1} A_{s}}, \cdots, \phi_{A_{s-1} A_{2 s-2}}, \rho_{A_{2 s-1}}\right), \quad n=2 s, s \geq 1, \\
& z=\frac{1}{2} \epsilon^{K L} v^{A_{1} \cdots A_{2 s-2}}\left(\phi_{A_{1} A_{s-1}}, \cdots, \phi_{A_{s-2} A_{2 s-4}} \phi_{A_{2 s-3} K} \phi_{A_{2 s-2} L}\right), \quad n=2 s-1, s \geq 2,
\end{aligned}
$$

where $u^{A_{1} \cdots A_{2 s-1}} \in\left(\bigvee^{s-1} \mathfrak{g}^{\vee} \otimes \mathfrak{g}^{\vee}\right)_{\text {ad } \vee \mathfrak{g}}$ totally symmetric in $A_{1}, \cdots, A_{2 s-1}, v^{A_{1} \cdots A_{2 s-2}} \in$ $\left(\bigvee^{s-2} \mathfrak{g}^{\vee} \otimes \bigwedge^{2} \mathfrak{g}^{\vee}\right)_{\text {ad }} \vee \mathfrak{g}$ totally symmetric in $A_{1}, \cdots, A_{2 s-2}$. Suppose now that $z \in \mathrm{w}_{\text {basic }}^{n, n+1} \cap$ $\cap_{A=1,2} \operatorname{ker} d_{A}$ so that $z$, besides being of the form $(5.2 .5 \mathrm{a}),(5.2 .5 \mathrm{~b})$, satisfies $d_{A} z=0$. Suppose first that $n=2 s$. Using (5.2.5a), (3.2.11f), (3.2.11h), the symmetry properties and the adg invariance of $u^{A_{1} \cdots A_{2 s-1}}$ and taking into account that terms with a different number of occurences of $\phi_{A B}, \rho_{A}$ are linearly independent, the condition $d_{A} z=0$ is equivalent to the equations

$$
\begin{aligned}
& \epsilon_{A A_{s-1}} u^{A_{1} \cdots A_{2 s-1}}\left(\phi_{A_{1} A_{s}}, \cdots, \phi_{A_{s-2} A_{2 s-3}}, \rho_{A_{2 s-2}}, \rho_{A_{2 s-1}}\right)=0 \\
& \frac{1}{2} \epsilon^{K L} u^{A_{1} \cdots A_{2 s-1}}\left(\phi_{A_{1} A_{s}}, \cdots, \phi_{A_{s-1} A_{2 s-2}},\left[\phi_{K A_{2 s-1}}, \phi_{L A}\right]\right)=0
\end{aligned}
$$

As $\rho_{A}$ is odd and $u^{A_{1} \cdots A_{2 s-1}}$ is totally symmetric in $A_{1}, \cdots, A_{2 s-1}$, (5.2.6a) entails that $u^{A_{1} \cdots A_{2 s-1}}$ is totally symmetric in its $s$ arguments. Using this fact and the ad $\mathfrak{g}$ invariance of $u^{A_{1} \cdots A_{2 s-1}}$, it is easy to see that $(5.2 .6 \mathrm{~b})$ is identically satisfied. Hence, $u^{A_{1} \cdots A_{2 s-1}} \in$ 
$\left(\bigvee^{s} \mathfrak{g}^{\vee}\right)_{\mathrm{ad}^{\vee} \mathfrak{g}}$. Conversely, if this holds, then (5.2.6a), (5.2.6b) are fulfilled. The above analysis shows that $\mathrm{w}_{\text {basic }}^{2 s, 2 s+1} \cap \cap_{A=1,2} \operatorname{ker} d_{A}$ is precisely the space of the $z$ of the form (5.2.5a) with $u^{A_{1} \cdots A_{2 s-1}} \in\left(\bigvee^{s} \mathfrak{g}^{\vee}\right)_{\mathrm{ad}^{\vee} \mathfrak{g}}$ totally symmetric in $A_{1}, \cdots, A_{2 s-1}$. Thus, we have a linear bijection $\mu: \mathrm{w}_{\text {basic }}^{2 s, 2 s+1} \cap \cap_{A=1,2} \operatorname{ker} d_{A} \mapsto\left(\bigvee^{s} \mathfrak{g}^{\vee}\right)_{\text {ad } \mathfrak{g}_{\mathfrak{g}}} \otimes \bigvee^{2 s-1} \mathbb{R}^{2}$ defined by $z \mapsto\left(u^{A_{1} \cdots A_{2 s-1}}\right)_{A_{1}, \cdots, A_{2 s-1}=1,2}$. We note next that $\mathrm{w}_{\text {basic }}^{2 s, 2 s-1}=0$. Indeed, if $z=r(\phi, \rho) \in$ $\mathrm{w}_{\text {basic }}^{n, n-1}$, the total number of internal indices $A=1,2$ and the total degree carried by $\phi_{A B}$, $\rho_{A}$ in each monomial of $r(\phi, \rho)$ must be $n-1+2 \nu$ and $n-1$, respectively, where $2 \nu$ is the number of indices contracted by means of $\epsilon^{A B}$. So, the numbers $m_{\phi}, m_{\rho}$ of occurences of $\phi_{A B}, \rho_{A}$ in a given monomial must satisfy the equations $2 m_{\phi}+1 m_{\rho}=n-1+2 \nu$, $2 m_{\phi}+3 m_{\rho}=n-1$. Taking into account that $m_{\phi}, m_{\rho}$ are non negative integers, one finds that there are no solutions for $n=2 s$ with $s>0$, so that $\mathrm{w}_{\text {basic }}^{2 s, 2 s-1}=0$ as announced. Thus, the bijection $\mu$ above induces a bijection $\hat{\mu}: H_{\text {basic }}^{2 s, 2 s+1}(\mathrm{w}) \mapsto\left(\bigvee^{s} \mathfrak{g}^{\vee}\right)_{\mathrm{ad}^{\vee} \mathfrak{g}} \otimes \bigvee^{2 s-1} \mathbb{R}^{2}$ Suppose next that $n=2 s-1$. Using (5.2.5b), (3.2.11f), (3.2.11h), the (anti)symmetry properties and the ad $\mathfrak{g}$ invariance of $v^{A_{1} \cdots A_{2 s-2}}$, the condition $d_{A} z=0$ is equivalent to the equation

$$
\begin{aligned}
& (s-2) \epsilon^{K L} \epsilon_{A A_{s-2}} v^{A_{1} \cdots A_{2 s-2}}\left(\phi_{A_{1} A_{s-1}}, \cdots, \phi_{A_{s-3} A_{2 s-5}}, \rho_{A_{2 s-4}}, \phi_{A_{2 s-3} K}, \phi_{A_{2 s-2} L}\right) \\
& +v^{A_{1} \cdots A_{2 s-2}}\left(\phi_{A_{1} A_{s-1}}, \cdots, \phi_{A_{s-2} A_{2 s-4}}, \rho_{A_{2 s-3}}, \phi_{A_{2 s-2} A}\right) \\
& +\epsilon^{K L} \epsilon_{A A_{2 s-3}} v^{A_{1} \cdots A_{2 s-2}}\left(\phi_{A_{1} A_{s-1}}, \cdots, \phi_{A_{s-2} A_{2 s-4}}, \rho_{K}, \phi_{A_{2 s-2} L}\right)=0 .
\end{aligned}
$$

Now, apply $u_{B}$ to this relation, using (3.2.11b), (3.2.11d), and then contract with $\epsilon^{B A}$. One gets then $\frac{1}{2} \epsilon^{K L} v^{A_{1} \cdots A_{2 s-2}}\left(\phi_{A_{1} A_{s-1}}, \cdots, \phi_{A_{s-2} A_{2 s-4}} \phi_{A_{2 s-3} K} \phi_{A_{2 s-2} L}\right)=0$. So, $z=0$. We conclude that $\mathrm{w}_{\text {basic }}^{2 s-1,2 s} \cap \cap_{A=1,2} \operatorname{ker} d_{A}=0$. Thus, $H_{\text {basic }}^{2 s-1,2 s}(\mathrm{w})=0$ as well.

5.3. The relation between the cohomologies of the $N=1$ and $N=2$ Weil superoperations

Let $\mathrm{w}(n)$ denote the $N=n$ Weil superoperations, $n=1,2$.

Corollary 7.3.1. One has

$$
\begin{aligned}
& H^{n, \pm n+1}(\mathrm{w}(2)) \simeq H^{ \pm\left(n-\frac{1}{2}\right)+\frac{1}{2}}(\mathrm{w}(1)) \otimes \mathrm{V}^{n-1} \mathbb{R}^{2}, \\
& H_{\text {basic }}^{n, \pm n+1}(\mathrm{w}(2)) \simeq H_{\text {basic }}^{ \pm\left(n-\frac{1}{2}\right)+\frac{1}{2}}(\mathrm{w}(1)) \otimes \bigvee^{n-1} \mathbb{R}^{2} .
\end{aligned}
$$

Proof. Combine props. 5.1.1, 5.2.1.

Thus, the $N=1$ and $N=2$ cohomologies of $\mathrm{w}$ are intimately related.

\section{Connections, equivariant cohomology and Weil homomorphism}

Let $\mathfrak{g}$ be an ungraded real Lie algebra.

6.1. The $N=1$ case

Let a be an $N=1 \mathfrak{g}$ superoperation with unity, i. e. a as an algebra has a unity 1 . 
Definition 6.1.1. A connection $a$ on a is an element of $a$ (3.1.10a), (3.1.12a), (3.1.12c) with $\omega$ substituted by a.

The curvature of $a$ is defined as usual as

$$
f=d a+\frac{1}{2}[a, a]
$$

It is easy to see that $f$ satisfies relations $(3.1 .10 \mathrm{~b}),(3.1 .12 \mathrm{~b}),(3.1 .12 \mathrm{~d})$ with $\phi$ substituted by $f$. In particular, being $j(\xi) f=0$ for any $\xi \in \mathfrak{g}, f$ is horizontal. $a, f$ together fulfill (3.1.11a), (3.1.11b).

We denote by Conn(a) the set of the connections of the $N=1 \mathfrak{g}$ superoperation a. $\operatorname{Conn}(\mathrm{a})$ is an affine space modelled on $\mathrm{a}^{1} \otimes \mathfrak{g}$.

Proposition 6.1.1. Let $r \in \bigwedge^{*} \mathfrak{g}^{\vee} \otimes \bigvee^{*} \mathfrak{g}^{\vee}$ be such that, for any connection $a \in$ Conn $(\mathrm{a}), r(a, f)$ is a representative of some element of $H_{\mathrm{basic}}^{p}(\mathrm{a})$ (see above eq. (5.1.3a) for the definition of the notation). Then, the basic cohomology class $[r(a, f)]$ is independent from the choice of a.

Proof. We follow the methods of ref. [32]. Consider the $N=1$ superoperation s generated by $s, \tilde{s}$ of degree $0,+1$, respectively, with

$$
\begin{gathered}
h^{s} s=0, \quad h^{s} \tilde{s}=\tilde{s}, \\
\tilde{h}^{s} s=-\tilde{s}, \quad \tilde{h}^{s} \tilde{s}=0, \\
i^{s}(\xi)=0, \quad \tilde{\imath}^{s}(\xi)=0, \quad \xi \in \mathfrak{g} .
\end{gathered}
$$

Next, we consider the graded tensor product superoperation $\mathbf{s} \hat{\otimes} \mathbf{a}$ and the subalgebra $\mathbf{c}$ of $\mathbf{s} \hat{\otimes} \mathbf{a}$ generated by the elements of the form $a(s), \tilde{h}^{s} a(s), \tilde{a}(s), \tilde{h}^{s} \tilde{a}(s)$, where $a: \mathbb{R} \mapsto \mathbf{a} \otimes \mathfrak{g}$ is a polynomial such that, for fixed $\sigma \in \mathbb{R}, a(\sigma)$ is a connection on a and $\tilde{a}(\sigma)=-\tilde{h} a(\sigma)$. Next, we define a degree 0 derivation $q$ on $c$ by

$$
\begin{aligned}
& q a(s)=0, \quad q \tilde{a}(s)=-\tilde{h}^{s} a(s), \\
& q \tilde{h}^{s} a(s)=0, \quad q \tilde{h}^{s} \tilde{a}(s)=0 .
\end{aligned}
$$

Note that, for fixed $\sigma \in \mathbb{R}, a(\sigma), \tilde{a}(\sigma)$ satisfy relations (3.1.3a)-(3.1.3d), (3.1.4a)-(3.1.4d) with $w, \tilde{w}$ replaced by $a(\sigma), \tilde{a}(\sigma)$. Using this fact, one easily checks that

$$
[q, \tilde{h}]=\tilde{h}^{s}, \quad\left[q, \tilde{h}^{s}\right]=0
$$




$$
\begin{gathered}
{\left[q, h+h^{s}\right]=0,} \\
{[q, i(\xi)]=0, \quad[q, \tilde{\imath}(\xi)]=0, \quad \xi \in \mathfrak{g} .}
\end{gathered}
$$

Let $r \in \bigwedge^{*} \mathfrak{g}^{\vee} \otimes \bigvee^{*} \mathfrak{g}^{\vee}$ be such that, for any connection $a$ on a, $r[a]:=r(a, \tilde{a})$ belongs to $\mathrm{a}_{\text {basic }} \cap \operatorname{ker} \tilde{h}$. By (6.1.5a) and the fact that $\tilde{h} r[a]=0$,

$$
\tilde{h}^{s} r[a(s)]=-\tilde{h} q r[a(s)] .
$$

We note that, by $(6.1 .2 \mathrm{c}),(6.1 .2 \mathrm{~d}),(6.1 .4 \mathrm{a}),(6.1 .4 \mathrm{~b}), \operatorname{qr}[a(s)]$ is necessarily of the form $\operatorname{qr}[a(s)]=\tilde{s} \alpha(s \mid a)$, where $\alpha(s \mid a)$ is a polynomial in $s$. From this expression and (6.1.2a), (6.1.2b), it follows that $h^{s} q r[a(s)]=q r[a(s)]$. By (6.1.6), one has then

$$
h q r[a(s)]=q(h-1) r[a(s)] .
$$

Further, from (6.1.7a), (6.1.7b) and the fact that $i(\xi) r[a]=0, \tilde{\imath}(\xi) r[a]=0$,

$$
i(\xi) \operatorname{qr}[a(s)]=0, \quad \tilde{\imath}(\xi) q r[a(s)]=0, \quad \xi \in \mathfrak{g} .
$$

For any element $x$ of $\mathbf{s} \hat{\otimes}$ a of the form $x=\tilde{s} \alpha(s)$ with $\alpha(s)$ a polynomials in $s$, we define $\int_{[0,1]} x=\int_{0}^{1} \alpha(\sigma) d \sigma$, where the right hand side is an ordinary Riemann integral. It is obvious that, for any element of $f(s)$ of $\mathbf{s} \hat{\otimes}$ a polynomial in $s, \tilde{h}^{s} f(s)$ is of the above form and $-\int_{[0,1]} \tilde{h}^{s} f(s)=f(1)-f(0)$. From (6.1.8), one has thus

$$
r[a(1)]-r[a(0)]=\tilde{h} \int_{[0,1]} q r[a(s)] .
$$

By (2.1.12b), the right hand side of (6.1.11) belongs to da. From (2.2.12a), (6.1.9), (6.1.10a), (6.1.10b), if $r[a]$ belongs to $\mathrm{a}_{\text {basic }}^{p}$ for any connection $a$ on a, then $\operatorname{qr}[a(\sigma)]$ belongs to $\mathrm{a}_{\text {basic }}^{p-1}$ for $\sigma \in \mathbb{R}$, so that $\int_{[0,1]} q r[a(s)]$ belongs to $\mathrm{a}_{\text {basic }}^{p-1}$, too.

Consider the $N=1$ Weil $\mathfrak{g}$ superoperation $w$ (cfr. subsect. 5.1). Then $\omega$ is a connection on w with curvature $\phi$ (cfr. eq. (3.1.9)).

Given an $N=1 \mathfrak{g}$ superoperation a with unity, one can define the graded tensor product $N=1 \mathfrak{g}$ superoperation $\mathbf{w} \hat{\otimes} \mathbf{a}$ (cfr. subsect. 4.1). The latter is the equivariant $N=1$ superoperation associated to a. The equivariant cohomology of a is, by definition the basic cohomology of $\mathbf{w} \hat{\otimes} \mathbf{a}$ :

$$
H_{\text {equiv }}^{p}(\mathrm{a})=H_{\text {basic }}^{p}(\mathrm{w} \hat{\otimes} \mathrm{a}), \quad p \in \mathbb{Z} .
$$

An equivariant cohomology class of a is represented by elements of $w \hat{\otimes} a$ of the form $r(\omega, \phi)$, where $r \in \Lambda^{*} \mathfrak{g}^{\vee} \otimes \bigvee^{*} \mathfrak{g}^{\vee} \otimes$ a. The Weil generator $\omega$ constitutes a connection of $\mathbf{w} \hat{\otimes} \mathbf{a}$. If $a$ is a connection of $\mathrm{a}, a$ is a connection of $\mathbf{w} \hat{\otimes} \mathbf{a}$ as well. By prop. 6.1.1, $r(\omega, \phi)$ is equivalent to $r(a, f)$ in equivariant cohomology. On the other hand, $r(a, f)$ is a representative of a basic cohomology class of a, which, by prop. 6.1.1, is independent from $a$ in basic cohomology. Thus, there is a natural homomorphism of $H_{\text {equiv }}^{*}\left(\right.$ a) into $H_{\text {basic }}^{p}($ a), called $N=1$ Weil homomorphism.

6.2. The $N=2$ case

Let a be an $N=2 \mathfrak{g}$ superoperation with unity. 
Definition 6.2.1. A connection $\left(a_{A}\right)_{A=1,2}$, on a is a doublet of a $\otimes \mathfrak{g}$ satisfying relations (3.2.10a), (3.2.10b), (3.2.11a), (3.2.12a), (3.2.12e), (3.2.12i) with $\omega_{A}$ substituted by $a_{A}$.

The derived connection

$$
b=\frac{1}{2} \epsilon^{K L} d_{K} a_{L}
$$

and the curvature and derived curvature

$$
\begin{aligned}
& f_{A B}=\frac{1}{2}\left(d_{A} a_{B}+d_{B} a_{A}+\left[a_{A}, a_{B}\right]\right) \\
& g_{A}=-\frac{1}{4} \epsilon^{K L} d_{K} d_{L} a_{A}-\frac{1}{2} \epsilon^{K L}\left[a_{K}, d_{L} a_{A}\right]-\frac{1}{6} \epsilon^{K L}\left[a_{K},\left[a_{L}, a_{A}\right]\right]
\end{aligned}
$$

satisfy relations (3.2.10c)-(3.2.10h), (3.2.12b)-(3.2.12d), (3.2.12f)-(3.2.12h), (3.2.12j)-(3. 2.12l) with $\gamma, \phi_{A B}, \rho_{A}$ substituted by $b, f_{A B}, g_{A}$, respectively. In particular, being $j(\xi) f_{A B}=0, j_{A}(\xi) f_{B C}=0, j(\xi) g_{A}=0, j_{A}(\xi) g_{B}=0$ for any $\xi \in \mathfrak{g}, f_{A B}, g_{A}$ are horizontal. $a_{A}, b, f_{A B}, g_{A}$ together satisfy (3.2.11b)-(3.2.11h).

We denote by Conn(a) the set of the connections of the $N=2$ superoperation a. Conn $(a)$ is an affine space modelled on $\mathrm{a}^{2,1} \otimes \mathfrak{g}$.

Proposition 6.2.1. Let $r \in \bigwedge^{*}\left(\mathfrak{g}^{\vee} \otimes \mathbb{R}^{2}\right) \otimes \bigvee^{*} \mathfrak{g}^{\vee} \otimes \bigvee^{*}\left(\mathfrak{g}^{\vee} \otimes \bigvee^{2} \mathbb{R}^{2}\right) \otimes \bigwedge^{*}\left(\mathfrak{g}^{\vee} \otimes \mathbb{R}^{2}\right)$ be such that, for any connection $\left(a_{A}\right)_{A=1,2} \in \operatorname{Conn}(\mathrm{a}), r(a, b, f, g)$ is a representative of some element of $H_{\text {basic }}^{n, p}(\mathrm{a})$ (see above eq. (5.2.3a) for the definition of the notation). Then, the basic cohomology class $[r(a, b, f, g)]$ is independent from the choice of $\left(a_{A}\right)_{A=1,2}$.

Proof. We generalize the methods of ref. [32]. Consider the $N=2$ superoperation s generated by $s, s, A, \tilde{s}$ of degree $0,+1,+2$, respectively, with

$$
\begin{array}{ll}
h^{s}{ }_{A} s=0, & h^{s}{ }_{A},_{, B}=0, \\
h^{s}{ }_{A} \tilde{s}=-s, A, & h^{s}{ }_{A, B} s=0, \\
h^{s}{ }_{A, C} s, B=-\epsilon_{B C} s, A, & h^{s}{ }_{A, B} \tilde{s}=-\epsilon_{A B} \tilde{s}, \\
\tilde{h}_{A}^{s} s=-s_{, A}, & \tilde{h}_{A}^{s}{ }_{, B}=\epsilon_{A B} \tilde{s}, \\
\tilde{h}_{A}^{s} \tilde{s}=0, & \\
i^{s}(\xi)=0, \quad i^{s}{ }_{A}(\xi)=0, & \tilde{i}^{s}(\xi)=0, \quad \xi \in \mathfrak{g} .
\end{array}
$$


Next, we consider the graded tensor product superoperation $\mathbf{s} \hat{\otimes} \mathbf{a}$ and the subalgebra $\mathbf{c}$ of $\mathbf{s} \hat{\otimes} \mathbf{a}$ generated by the elements of the form $a_{A}(s), \tilde{h}^{s}{ }_{A} a_{B}(s), \tilde{h}^{s} \tilde{h}^{s}{ }_{B} a_{C}(s), a_{A, B}(s)$, $\tilde{h}^{s}{ }_{A} a_{B, C}(s), \tilde{h}_{A}^{s} \tilde{h}_{B}^{s} a_{C, D}(s), \tilde{a}_{A}(s), \tilde{h}^{s}{ }_{A} \tilde{a}_{B}(s), \tilde{h}^{s}{ }_{A} \tilde{h}^{s}{ }_{B} \tilde{a}_{C}(s)$, where $a_{A}: \mathbb{R} \mapsto \mathrm{a} \otimes \mathfrak{g}$, $A=1,2$, is a polynomial such that, for fixed $\sigma \in \mathbb{R}, a_{A}(\sigma)$ is a connection of a and $a_{A, B}(\sigma)=-\tilde{h}_{B} a_{A}(\sigma), \tilde{a}_{A}(\sigma)=\frac{1}{2} \epsilon^{K L} \tilde{h}_{K} \tilde{h}_{L} a_{A}(\sigma)$. Next, we define a degree 0 derivation $q$ on $\mathrm{c}$ by

$$
\begin{array}{ll}
q a_{A}(s)=0, & q a_{A, B}(s)=-\tilde{h}_{B}^{s} a_{A}(s), \\
q \tilde{a}_{A}(s)=-\epsilon^{K L} \tilde{h}^{s}{ }_{K} a_{A, L}(s), & q \tilde{h}^{s}{ }_{A} a_{B}(s)=0, \\
q \tilde{h}^{s}{ }_{A} a_{B, C}(s)=\epsilon_{A C} \frac{1}{2} \epsilon^{K L} \tilde{h}^{s}{ }_{K} \tilde{h}^{s}{ }_{L} a_{B}(s), & q \tilde{h}^{s}{ }_{A} \tilde{a}_{B}(s)=\frac{1}{2} \epsilon^{K L} \tilde{h}^{s}{ }_{K} \tilde{h}^{s}{ }_{L} a_{B, A}(s), \\
q \frac{1}{2} \epsilon^{K L} \tilde{h}^{s}{ }_{K} \tilde{h}^{s}{ }_{L} a_{A}(s)=0, & q \frac{1}{2} \epsilon^{K L} \tilde{h}^{s}{ }_{K} \tilde{h}^{s}{ }_{L} a_{A, B}(s)=0, \\
q \frac{1}{2} \epsilon^{K L} \tilde{h}^{s}{ }_{K} \tilde{h}^{s}{ }_{L} \tilde{a}_{A}(s)=0 . &
\end{array}
$$

Note that, for fixed $\sigma \in \mathbb{R}, a(\sigma), a_{A}(\sigma), \tilde{a}(\sigma)$ satisfy relations (3.2.3a)-(3.2.3i), (3.2.4a)(3.2.4i) with $w, w_{A}, \tilde{w}$ replaced by $a(\sigma), a_{A}(\sigma), \tilde{a}(\sigma)$. Using this fact, one easily checks that

$$
\begin{gathered}
{\left[q, \tilde{h}_{A}\right]=\tilde{h}_{A}^{s}, \quad\left[q, \tilde{h}_{A}^{s}\right]=0,} \\
{\left[q, h_{A, B}+h_{A, B}^{s}\right]=0,} \\
{[q, i(\xi)]=0, \quad\left[q, i_{, A}(\xi)\right]=0, \quad[q, \tilde{\imath}(\xi)]=0, \quad \xi \in \mathfrak{g} .}
\end{gathered}
$$

Using (6.2.6a), it is easy to show that

$$
\left[q, \frac{1}{2} \epsilon^{K L} \tilde{h}_{K} \tilde{h}_{L}\right]=\epsilon^{K L} \tilde{h}_{K}^{s} \tilde{h}_{L}, \quad \frac{1}{2}\left[q,\left[q, \frac{1}{2} \epsilon^{K L} \tilde{h}_{K} \tilde{h}_{L}\right]\right]=\frac{1}{2} \epsilon^{K L} \tilde{h}_{K}^{s} \tilde{h}_{L}^{s}
$$

Let $r \in \Lambda^{*}\left(\mathfrak{g}^{\vee} \otimes \mathbb{R}^{2}\right) \otimes \bigvee^{*}\left(\mathfrak{g}^{\vee} \otimes \bigotimes^{2} \mathbb{R}^{2}\right) \otimes \Lambda^{*}\left(\mathfrak{g}^{\vee} \otimes \mathbb{R}^{2}\right)$ be such that, for any connection $a_{A}$, $A=1,2$, on a, $r[a]:=r(a, a, \tilde{a})$ belongs to $a_{\text {basic }} \cap \cap_{A=1,2} \operatorname{ker} \tilde{h}_{A}$. Using (6.2.9a), (6.2.9b) and the fact that $\tilde{h}_{A} r[a]=0$, it is easy to see that

$$
\frac{1}{2} \epsilon^{K L} \tilde{h}_{K}^{s} \tilde{h}_{L}^{s} r[a(s)]=\frac{1}{2} \epsilon^{K L} \tilde{h}_{K} \tilde{h}_{L} \frac{1}{2} q^{2} r[a(s)]
$$

We note that, by $(6.2 .3 \mathrm{~g})-(6.2 .3 \mathrm{i}),(6.2 .5 \mathrm{a})-(6.2 .5 \mathrm{f}), \frac{1}{2} q^{2} r[a(s)]$ is necessarily of the form $\frac{1}{2} q^{2} r[a(s)]=\tilde{s} \alpha(s \mid a)+\frac{1}{2} \epsilon^{K L} s_{, K}{ }_{, L} \beta(s \mid a)$, where $\alpha(s \mid a), \beta(s \mid a)$ are polynomials in $s$. From 
this expression and (6.2.3d)-(6.2.3f), it follows that $h^{s}{ }_{A, B} \frac{1}{2} q^{2} r[a(s)]=-\epsilon_{A B} \frac{1}{2} q^{2} r[a(s)]$. By (6.2.7), one has then

$$
h_{A, B} \frac{1}{2} q^{2} r[a(s)]=\frac{1}{2} q^{2}\left(h_{A, B}+\epsilon_{A B}\right) r[a(s)] .
$$

Further, from (6.2.8a)-(6.2.8c) and the fact that $i(\xi) r[a]=0, i_{, A}(\xi) r[a]=0, \tilde{\imath}(\xi) r[a]=0$,

$$
i(\xi) \frac{1}{2} q^{2} r[a(s)]=0, \quad i_{, A}(\xi) \frac{1}{2} q^{2} r[a(s)]=0, \quad \tilde{\imath}(\xi) \frac{1}{2} q^{2} r[a(s)]=0, \quad \xi \in \mathfrak{g}
$$

For any element $x$ of $\mathbf{s} \hat{\otimes} \mathbf{a}$ of the form $x=\tilde{s} \alpha(s)+\frac{1}{2} \epsilon^{K L} s_{, K} s_{, L} \beta(s)$ with $\alpha(s), \beta(s)$ polynomials in $s$, we define $\int_{[0,1]} x=\int_{0}^{1} \alpha(\sigma) d \sigma$, where the right hand side is an ordinary Riemann integral. It is not difficult to show that, for any element of $f(s)$ of $\mathbf{s} \hat{\otimes}$ a polynomial in $s$, $\frac{1}{2} \epsilon^{K L} \tilde{h}_{K}^{s} \tilde{h}_{L}^{s} f(s)$ is of the above form and $\int_{[0,1]} \frac{1}{2} \epsilon^{K L} \tilde{h}_{K}^{s} \tilde{h}_{L}^{s} f(s)=f(1)-f(0)$. From $(6.2 .10)$,

$$
r[a(1)]-r[a(0)]=\frac{1}{2} \epsilon^{K L} \tilde{h}_{K} \tilde{h}_{L} \int_{[0,1]} \frac{1}{2} q^{2} r[a(s)] .
$$

By $(2.2 .14 \mathrm{~d})$, the right hand side of (6.2.13) belongs to $\frac{1}{2} \epsilon^{K L} d_{K} d_{L}$ a. From (2.2.14a), $(2.2 .14 \mathrm{~b}),(6.2 .11),(6.2 .12 \mathrm{a})-(6.2 .12 \mathrm{c})$, if $r[a]$ belongs to $a_{\mathrm{basic}}^{n, p}$ for any connection $a_{A}$ on a, then $\frac{1}{2} q^{2} r[a(\sigma)]$ belongs to $a_{\text {basic }}^{n, p-2}$ for $\sigma \in \mathbb{R}$, so that $\int_{[0,1]} \frac{1}{2} q^{2} r[a(s)]$ belongs to $a_{\text {basic }}^{n, p-2}$, too.

Consider the $N=2$ Weil $\mathfrak{g}$ superoperation $\mathrm{w}$ (cfr. subsect. 5.2). Then, $\omega_{A}$ is a connection of $\mathrm{w}$ with derived connection $\gamma$ and curvature and derived curvature $\phi_{A B}, \rho_{A}$ (cfr. eqs. (3.2.9a) $-(3.2 .9 \mathrm{~b}))$.

Given an $N=2 \mathfrak{g}$ superoperation a, one can define the graded tensor product $N=2 \mathfrak{g}$ superoperation $\mathbf{w} \hat{\otimes} \mathbf{a}$ (cfr. subsect. 4.2). The latter is the equivariant $N=2$ superoperation associated to a. The equivariant cohomology of a is by definition the basic cohomology of w $\hat{\otimes} \mathrm{a}:$

$$
H_{\text {equiv }}^{n, p}(\mathrm{a})=H_{\text {basic }}^{n, p}(\mathbf{w} \hat{\otimes} \mathrm{a}), \quad(n, p) \in \mathbb{N} \times \mathbb{Z} .
$$

An equivariant cohomology class of a is represented by elements of $w \hat{\otimes} a$ of the form $r(\omega, \gamma, \phi, \rho)$, where $r \in \Lambda^{*}\left(\mathfrak{g}^{\vee} \otimes \mathbb{R}^{2}\right) \otimes \bigvee^{*} \mathfrak{g}^{\vee} \otimes \bigvee^{*}\left(\mathfrak{g}^{\vee} \otimes \bigvee^{2} \mathbb{R}^{2}\right) \otimes \Lambda^{*}\left(\mathfrak{g}^{\vee} \otimes \mathbb{R}^{2}\right) \otimes$ a. The Weil generator $\omega_{A}$ constitutes a connection of $\mathbf{w} \hat{\otimes} \mathbf{a}$. If $a_{A}$ is a connection of $\mathrm{a}, a_{A}$ is a connection of $\mathbf{w} \hat{\otimes} \mathbf{a}$ as well. By prop. $6.2 .1, r(\omega, \gamma, \phi, \rho)$ is equivalent to $r(a, b, f, g)$ in equivariant cohomology. On the other hand, $r(a, b, f, g)$ is a representative of a basic cohomology class of a, which, by prop. 6.2.1, is independent from $a_{A}$ in basic cohomology. Thus, there is a natural homomorphism of $H_{\text {equiv }}^{n, p}\left(\right.$ a) into $H_{\text {basic }}^{n, p}($ a), called $N=2$ Weil homomorphism. 


\section{Superoperations of a smooth manifold with a group action}

Let $M$ be a smooth $m$ dimensional real manifold. Thus, $M$ is endowed with a collection of smooth charts $\left(U_{a}, x_{a}\right), a \in A$, in the usual way. Let $M$ carry the right action of a real Lie group $G$ with Lie algebra $\mathfrak{g}$ (see ref. [33] for an exhaustive treatment of the theory of manifolds with a group action).

Let $\mathrm{s}$ be a real Grassmann algebra such that $\mathrm{s}^{0} \simeq \mathbb{R}$.

\section{1. $N=1$ differential geometry}

Definition 7.1.1. An $N=1$ differential structure on $M$ is a collection $\left\{\left(U_{a}, X_{a}\right) \mid a \in\right.$ A\}, where

i) $\left\{U_{a} \mid a \in A\right\}$ is an open covering of $M$;

ii) for each $a \in A, X_{a}: U_{a} \mapsto\left(\mathrm{S}_{1}^{0}\right)^{m}$ and $x_{a}=\left.X_{a}\right|_{\theta=0}: U_{a} \mapsto \mathbb{R}^{m}$ is a coordinate of $M$;

iii) for $a, b \in A$ such that $U_{a} \cap U_{b} \neq \emptyset, X_{a}=x_{a} \circ x_{b}{ }^{-1}\left(X_{b}\right)$.

Below, we shall omit the chart indices $a, b, \ldots$ except when dealing with matching relations.

We write as usual

$$
X^{i}=x^{i}+\theta \tilde{x}^{i}, \quad \tilde{X}^{i}=\tilde{x}^{i},
$$

where $x^{i}: U \mapsto \mathbb{R}, \tilde{x}^{i}: U \mapsto \mathrm{s}^{1}$.

We introduce the $N=1$ covariant superderivatives

$$
D_{i}=\tilde{\partial}_{x i}+\theta \partial_{x i}, \quad \tilde{D}_{i}=\partial_{x i},
$$

where $\tilde{\partial}_{x i}=\partial / \partial \tilde{x}^{i}$. One has relations

$$
\begin{aligned}
& {\left[D_{i}, D_{j}\right]=0, \quad\left[D_{i}, \tilde{D}_{j}\right]=0,} \\
& {\left[\tilde{D}_{i}, \tilde{D}_{j}\right]=0}
\end{aligned}
$$

Further,

$$
\begin{array}{ll}
D_{i} X^{j}=0, & D_{i} \tilde{X}^{j}=\delta_{i}^{j}, \\
\tilde{D}_{i} X^{j}=\delta_{i}^{j}, & \tilde{D}_{i} \tilde{X}^{j}=0 .
\end{array}
$$

Using (7.1.1a), (7.1.1b), it is straightforward to check that relations (7.1.4a)-(7.1.4d) completely characterize $D_{i}, \tilde{D}_{i}$. 
The transformation properties of $X^{i}$ under chart changes, stated in def. 7.1.1, imply that

$$
\tilde{X}_{a}{ }^{i}=\tilde{X}_{b}{ }^{j} \tilde{D}_{b j} X_{a}{ }^{i} .
$$

Using that (7.1.4a)-(7.1.4d) completely characterize $D_{i}, \tilde{D}_{i}$, one can show easily that they match as

$$
D_{a i}=\tilde{D}_{a i} X_{b}^{j} D_{b j}, \quad \tilde{D}_{a i}=\tilde{D}_{a i} \tilde{X}_{b}^{j} D_{b j}+\tilde{D}_{a i} X_{b}{ }^{j} \tilde{D}_{b j} .
$$

We denote by $\mathcal{F}$ the sheaf of germs of smooth $N=1$ functions on $M$ generated by $X^{i}, \tilde{X}^{i}$. By definition, a generic element $F \in \mathcal{F}(U)$ is a finite sum of the form $F=$ $\sum_{p \geq 0} f_{i_{1} \cdots i_{p}} \circ X \tilde{X}^{i_{1}} \cdots \tilde{X}^{i_{p}}$ for certain smooth maps $f_{i_{1} \cdots i_{p}}: \mathbb{R}^{m} \mapsto \mathbb{R}$ antisymmetric in $i_{1}, \cdots, i_{p}$. It is easy to see that

$$
F=\sum_{p=0}^{m}\left[F_{i_{1} \cdots i_{p}}+\theta \partial_{x i_{0}} F_{i_{i} \cdots i_{p}} \tilde{x}^{i_{0}}\right] \tilde{x}^{i_{1}} \cdots \tilde{x}^{i_{p}},
$$

where $F_{i_{1} \cdots i_{p}}=f_{i_{1} \cdots i_{p}} \circ x . \mathcal{F}$ has a natural grading corresponding to the total s degree of $\tilde{x}^{i}$.

We define on $U_{a} \cap U_{b} \neq \emptyset$,

$$
Z_{a b}{ }^{i}{ }_{j}=\tilde{D}_{b j} X_{a}{ }^{i} .
$$

It is easy to see that $Z$ is a $\operatorname{GL}(m, \mathcal{F}) 1$-cocycle on $M . Z$ is called the fundamental 1-cocycle of the $N=1$ differential structure. One can introduce in standard fashion the sheaf $\mathcal{F}_{r, s}:=\mathcal{F}\left(Z^{\otimes r} \otimes Z^{\vee \otimes s}\right)$ of germs of smooth $N=1$ sections of $Z^{\otimes r} \otimes Z^{\vee \otimes s}$. We denote by $\mathrm{f}_{\theta r, s}$ the real vector space of sections of $\mathcal{F}_{r, s}$ on $M$.

Notice that $\mathrm{f}_{\theta r, s} \simeq \mathrm{f}_{0 r, s}$, since, by (7.1.7), any $F \in \mathcal{F}(U)$ is completely determined by $f=\left.F\right|_{\theta=0}$.

$z=\left.Z\right|_{\theta=0}$ is nothing but the tangent bundle 1-cocycle of $M$. By (7.1.1b), (7.1.5), $\mathrm{f}_{0 r, s}{ }^{p}$, and thus also $\mathrm{f}_{\theta r, s}{ }^{p}$ by the previous remark, can be identified with the space of degree $p$ smooth tensor fields of type $r, s$ on $M$.

We are particularly interested in the space $\mathrm{f}_{\theta 0,0}$, which is a graded algebra.

We define

$$
H=\tilde{X}^{i} D_{i}, \quad \tilde{H}=-\tilde{X}^{i} \tilde{D}_{i} .
$$

Using (7.1.5), (7.1.6a), (7.1.6b), it is easy to see that $H, \tilde{H}$ are globally defined derivations on $\mathrm{f}_{\theta 0,0}$.

Denoting by $c \xi$ the fundamental vector field on $M$ induced by $\xi \in \mathfrak{g}$, we define further

$$
I(\xi)=C^{i} \xi D_{i}, \quad \tilde{I}(\xi)=C^{i} \xi \tilde{D}_{i}+\tilde{X}^{j} \tilde{D}_{j} C^{i} \xi D_{i}
$$


where $C \xi$ is the element of $\mathrm{f}_{\theta 1,0}{ }^{0}$ corresponding to $c \xi$ given explicitly by $C^{i} \xi=c^{i} \xi+$ $\theta \tilde{x}^{j} \partial_{x j} c^{i} \xi$. By (7.1.6a), (7.1.8), $I(\xi), \tilde{I}(\xi)$ are also globally defined derivations on $\mathrm{f}_{\theta 0,0}$.

Using the relation $D_{i} C^{j} \xi=0$, it is now straightforward to verify that $H, \tilde{H}, I, \tilde{I}$ satisfy relations $(2.1 .2 \mathrm{a})-(2.1 .2 \mathrm{c}),(2.1 .5 \mathrm{a})-(2.1 .5 \mathrm{c}),(2.1 .8 \mathrm{a})-(2.1 .8 \mathrm{~d})$. In this way, $\mathrm{f}_{\theta 0,0}$ becomes a $\mathbb{Z}$ graded real left module algebra of the $\mathbb{Z}$ graded real Lie algebra $\mathrm{t}_{\theta}$ (cfr. sect 2.1).

Thus, $f:=f_{00,0}$ acquires the structure of $N=1 \mathfrak{g}$ superoperation (cfr. def. 4.1.1), the relevant graded derivations being

$$
\begin{gathered}
h=\tilde{x}^{i} \tilde{\partial}_{x i}, \quad \tilde{h}=-\tilde{x}^{i} \partial_{x i} \\
i(\xi)=c^{i} \xi \tilde{\partial}_{x i}, \quad \tilde{\imath}(\xi)=c^{i} \xi \partial_{x i}+\tilde{x}^{j} \partial_{x j} c^{i} \xi \tilde{\partial}_{x i} .
\end{gathered}
$$

This superoperation is canonically associated to the $N=1$ differential structure.

Now, from (7.1.7) and (7.1.5), it appears that the graded algebra $f$ is isomorphic to the graded algebra of ordinary differential forms on $M$. Under such an isomorphism, the derivations $k, d, j(\xi), l(\xi)$, defined in (2.1.12a), (2.1.12b), (2.1.13a), (2.1.13b), correspond to the form degree $k_{\mathrm{dR}}$, the de Rham differential $d_{\mathrm{dR}}$, the contraction $j_{\mathrm{dR}}(\xi)$ and the Lie derivative $l_{\mathrm{dR}}(\xi)$, respectively. Therefore, the above is nothing but a reformulation of the customary theory of differential forms, so that, in particular, the (basic) cohomology of $f$ is isomorphic to the (basic) de Rham cohomology.

Theorem 7.1.1. There is an isomorphism of the $N=1$ (basic) cohomology of $\mathrm{f}$ the de Rham (basic) cohomology of the $(G)$ manifold $M$. Indeed, one has that $H^{p}(\mathrm{f})=0$ $\left(H_{\text {basic }}^{p}(\mathbf{f})=0\right)$, except perhaps for $0 \leq p \leq m$, and

$$
\begin{gathered}
H^{p}(\mathrm{f}) \simeq H_{\mathrm{dR}}^{p}(M), \quad 0 \leq p \leq m, \\
H_{\text {basic }}^{p}(\mathrm{f}) \simeq H_{\mathrm{dR} \text { basic }}^{p}(M) \quad 0 \leq p \leq m .
\end{gathered}
$$

Proof. See the above remarks.

Recall that a connection $y$ on the $G$ space $M$ is a $\mathfrak{g}$ valued 1 form satisfying relations (3.1.10a), (3.1.12a), (3.1.12c) with $j, l, \omega$ substituted by $j_{\mathrm{dR}}, l_{\mathrm{dR}}, y$ respectively [33]. We denote by Conn $(M)$ the affine space of the connections on $M$.

Theorem 7.1.2. One has

$$
\operatorname{Conn}(\mathfrak{f}) \simeq \operatorname{Conn}(M)
$$

(cfr. def. 6.1.1). 
Proof. Any $a \in \mathrm{f}^{1} \otimes \mathfrak{g}$ is locally of the form $a=a_{i} \tilde{x}^{i}$, where $a_{i}$ is a $\mathfrak{g}$ valued smooth map. Define $\lambda(a)=a_{i} d_{\mathrm{dR}} x^{i}$. Then, by the above remarks, $\lambda(a)$ is a connection of $M$ if and only if $a$ is a connection of $\mathrm{f}$. The map $\lambda$ is obviously a bijection.

\section{2. $N=2$ differential geometry}

Definition 7.2.1. An $N=2$ differential structure on $M$ is a collection $\left\{\left(U_{a}, X_{a}\right) \mid a \in\right.$ A\}, where

i) $\left\{U_{a} \mid a \in A\right\}$ is an open covering of $M$;

ii) for each $a \in A, X_{a}: U_{a} \mapsto\left(S_{2}^{0}\right)^{m}$ and $x_{a}=\left.X_{a}\right|_{\theta=0}: U_{a} \mapsto \mathbb{R}^{m}$ is a coordinate of $M$; iii) for $a, b \in A$ such that $U_{a} \cap U_{b} \neq \emptyset, X_{a}=x_{a} \circ x_{b}{ }^{-1}\left(X_{b}\right)$.

Below, we shall omit the chart indices $a, b, \ldots$ except when dealing with matching relations.

We write as usual

$$
\begin{aligned}
& X^{i}=x^{i}+\theta^{A} x^{i}{ }_{, A}+\frac{1}{2} \epsilon_{K L} \theta^{K} \theta^{L} \tilde{x}^{i}, \quad X^{i}{ }_{, A}=x^{i}{ }_{, A}+\epsilon_{A K} \theta^{K} \tilde{x}^{i}, \\
& \tilde{X}^{i}=\tilde{x}^{i},
\end{aligned}
$$

where $x^{i}: U \mapsto \mathbb{R}, x^{i}, A: U \mapsto \mathrm{s}^{1}, \tilde{x}^{i}: U \mapsto \mathrm{s}^{2}$.

We introduce the $N=2$ covariant superderivatives

$$
\begin{aligned}
& D_{i}=\tilde{\partial}_{x i}+\epsilon_{K L} \theta^{K} \partial_{x i}{ }^{L}+\frac{1}{2} \epsilon_{K L} \theta^{K} \theta^{L} \partial_{x i}, \quad D_{i, A}=\epsilon_{A K}\left(\partial_{x i}{ }^{K}+\theta^{K} \partial_{x i}\right), \\
& \tilde{D}_{i}=\partial_{x i}
\end{aligned}
$$

where $\partial_{x i}{ }^{A}=\partial / \partial x^{i}{ }_{, A}, \tilde{\partial}_{x i}=\partial / \partial \tilde{x}^{i}$. One has

$$
\begin{aligned}
& {\left[D_{i}, D_{j}\right]=0, \quad\left[D_{i}, D_{j, A}\right]=0, \quad\left[D_{i}, \tilde{D}_{j}\right]=0,} \\
& {\left[D_{i, A}, D_{j, B}\right]=0, \quad\left[D_{i, A}, \tilde{D}_{j}\right]=0, \quad\left[\tilde{D}_{i}, \tilde{D}_{j}\right]=0 .}
\end{aligned}
$$

Further,

$$
\begin{array}{lll}
D_{i} X^{j}=0, & D_{i} X^{j}{ }_{, A}=0, & D_{i} \tilde{X}^{j}=\delta_{i}^{j}, \\
D_{i, A} X^{j}=0, & D_{i, A} X^{j}{ }_{, B}=\epsilon_{A B} \delta_{i}^{j}, & D_{i, A} \tilde{X}^{j}=0, \\
\tilde{D}_{i} X^{j}=\delta_{i}^{j}, & \tilde{D}_{i} X^{j}{ }_{, A}=0, & \tilde{D}_{i} \tilde{X}^{j}=0 .
\end{array}
$$


By (7.2.1a)-(7.2.1c), relations (7.2.4a)-(7.2.4i) completely characterize $D_{i}, D_{i, A}, \tilde{D}_{i}$.

The transformation properties of $X^{i}$ under chart changes, stated in def. 7.2.1, imply that

$$
X_{a}{ }^{i}, A=X_{b}{ }^{j}{ }_{A} \tilde{D}_{b j} X_{a}{ }^{i}, \quad \tilde{X}_{a}{ }^{i}=\tilde{X}_{b}{ }^{j} \tilde{D}_{b j} X_{a}{ }^{i}+\frac{1}{2} \epsilon^{J K} X_{b}{ }^{j}{ }_{J} X_{b}{ }^{k}{ }_{, K} \tilde{D}_{b j} \tilde{D}_{b k} X_{a}{ }^{i} .
$$

Using that (7.2.4a)-(7.2.4i) completely characterize $D_{i}, D_{i, A}, \tilde{D}_{i}$, one can show easily that they match as

$$
\begin{aligned}
& D_{a i}=\tilde{D}_{a i} X_{b}{ }^{j} D_{b j}, \quad D_{a i, A}=\tilde{D}_{a i} X_{b}{ }^{j}{ }_{, A} D_{b j}+\tilde{D}_{a i} X_{b}{ }^{j} D_{b j, A}, \\
& \tilde{D}_{a i}=\tilde{D}_{a i} \tilde{X}_{b}{ }^{j} D_{b j}+\epsilon^{K L} \tilde{D}_{a i} X_{b}{ }^{k}{ }_{K} D_{b k, L}+\tilde{D}_{a i} X_{b}{ }^{j} \tilde{D}_{b j} .
\end{aligned}
$$

We denote by $\mathcal{F}$ the sheaf of germs of smooth $N=2$ functions on $M$ generated by $X^{i}, X^{i}{ }_{A}, \tilde{X}^{i}$. By definition, a generic element $F \in \mathcal{F}(U)$ is a finite sum of the form $F=\sum_{p, q \geq 0} f_{i_{1} \cdots i_{p} i_{p+1} \cdots i_{p+q}}^{I_{1} \cdots I_{p}} \circ X X^{i_{1}}{ }_{I_{1}} \cdots X^{i_{p}}, I_{p} \tilde{X}^{i_{p+1}} \cdots \tilde{X}^{i_{p+q}}$ for certain smooth maps $f_{i_{1} \cdots i_{p} i_{p+1} \cdots i_{p+q}}^{I_{1} \cdots I_{p}}: \mathbb{R}^{m} \mapsto \mathbb{R}$ antisymmetric in the pairs $\left(i_{1}, I_{1}\right), \cdots,\left(i_{p}, I_{p}\right)$ and symmetric in $i_{p+1}, \cdots, i_{p+q}$. It is straightforward though tedious to show that

$$
\begin{aligned}
& F=\sum_{p=0}^{2 m} \sum_{q=0}^{q_{0}}\left\{F_{i_{1} \cdots i_{p} i_{p+1} \cdots i_{p+q}}^{I_{1} \cdots I_{p}} x^{i_{1}}{ }_{, I_{1}} x^{i_{2}}, I_{2}\right. \\
& +\theta^{K}\left[\delta_{K}^{I_{0}} \partial_{i_{0}} F_{i_{1} \cdots i_{p} i_{p+1} \cdots i_{p+q}}^{I_{1} \cdots I_{p}} x^{i_{0}}{ }_{, I_{0}} x^{i_{1}}{ }_{, I_{1}} x^{i_{2}}{ }_{, I_{2}}-p \epsilon_{K I_{1}} F_{i_{1} \cdots i_{p} i_{p+1} \cdots i_{p+q}}^{I_{1} \cdots I_{p}} x^{i_{2}}{ }_{, I_{2}} \tilde{x}^{i_{1}}\right] \\
& +\frac{1}{2} \epsilon_{K L} \theta^{K} \theta^{L}\left[\frac{1}{2} \epsilon^{I_{-1} I_{0}} \partial_{i_{-1}} \partial_{i_{0}} F_{i_{1} \cdots i_{p} i_{p+1} \cdots i_{p+q}}^{I_{1} \cdots I_{p}} x^{i_{-1}}{ }_{, I_{-1}} x^{i_{0}}{ }_{, I_{0}} x^{i_{1}}{ }_{, I_{1}} x^{i_{2}}, I_{2}\right. \\
& +\partial_{i_{0}} F_{i_{1} \cdots i_{p} i_{p+1} \cdots i_{p+q}}^{I_{1} \cdots I_{p}} x^{i_{1}}{ }_{, I_{1}} x^{i_{2}}{ }_{, I_{2}} \tilde{x}^{i_{0}}-p \delta_{I_{1}}^{I_{0}} \partial_{i_{0}} F_{i_{1} \cdots i_{p} i_{p+1} \cdots i_{p+q}}^{I_{1} \cdots I_{p}} x^{i_{0}}{ }_{I_{0}} x^{i_{2}}{ }_{, I_{2}} \tilde{x}^{i_{1}} \\
& \left.\left.+\frac{1}{2} p(p-1) \epsilon_{I_{1} I_{2}} F_{i_{1} \cdots i_{p} i_{p+1} \cdots i_{p+q}}^{I_{1} \cdots I_{p}} \tilde{x}^{i_{1}} \tilde{x}^{i_{2}}\right]\right\} x^{i_{3}}{ }_{, I_{3}} \cdots x^{i_{p}}, I_{p} \tilde{x}^{i_{p+1}} \cdots \tilde{x}^{i_{p+q}},
\end{aligned}
$$

where $F_{i_{1} \cdots i_{p} i_{p+1} \cdots i_{p+q}}^{I_{1} \cdots I_{p}}=f_{i_{1} \cdots i_{p} i_{p+1} \cdots i_{p+q}}^{I_{1} \cdots I_{p}} \circ x . \mathcal{F}$ has a natural grading corresponding to the total s degree of $x^{i}{ }_{, I}, \tilde{x}^{i}$.

We define on $U_{a} \cap U_{b} \neq \emptyset$,

$$
Z_{a b}{ }^{i}{ }_{j}=\tilde{D}_{b j} X_{a}{ }^{i} .
$$


It is easy to see that $Z$ is a $\operatorname{GL}(m, \mathcal{F}) 1$-cocycle on $M . Z$ is called the fundamental 1 cocycle of the $N=2$ differential structure. One can introduce in standard fashion the sheaf $\mathcal{F}_{r, s}:=\mathcal{F}\left(Z^{\otimes r} \otimes Z^{\vee \otimes s}\right)$ of germs of smooth $N=2$ sections of $Z^{\otimes r} \otimes Z^{\vee \otimes s}$. We denote by $\mathrm{f}_{\theta r, s}$ the real vector space of sections of $\mathcal{F}_{r, s}$ on $M$.

Notice that $\mathrm{f}_{\theta r, s} \simeq \mathrm{f}_{0 r, s}$, since, by (7.2.7), any $F \in \mathcal{F}(U)$ is completely determined by $f=\left.F\right|_{\theta=0}$.

$z=\left.Z\right|_{\theta=0}$ is nothing but the tangent bundle 1-cocycle of $M$. However, unlike the $N=1$ case, there is no simple geometrical interpretation of the spaces $\mathrm{f}_{0 r, s}{ }^{p}, \mathrm{f}_{\theta r, s} p$.

We are particularly interested in the space $\mathrm{f}_{\theta 0,0}$, which is a graded algebra.

We define

$$
\begin{aligned}
& H_{A}=-X^{i}{ }_{, A} D_{i}, \quad H_{A, B}=X^{i}{ }_{, A} D_{i, B}-\epsilon_{A B} \tilde{X}^{i} D_{i} \\
& \tilde{H}_{A}=\tilde{X}^{i} D_{i, A}-X^{i}{ }_{, A} \tilde{D}_{i} .
\end{aligned}
$$

Using (7.2.5a), (7.2.5b), (7.2.6a)-(7.2.6c), it is easy to see that $H_{A}, H_{A, B}, \tilde{H}_{A}$ are globally defined derivations on $\mathrm{f}_{\theta 0,0}$.

We set next

$$
\begin{aligned}
& I(\xi)=C^{i} \xi D_{i}, \quad I_{, A}(\xi)=X^{j}{ }_{, A} \tilde{D}_{j} C^{i} \xi D_{i}+C^{i} \xi D_{i, A} \\
& \tilde{I}(\xi)=\left[\tilde{X}^{j} \tilde{D}_{j} C^{i} \xi+\frac{1}{2} \epsilon^{K L} X^{k}{ }_{, K} X^{l}{ }_{, L} \tilde{D}_{k} \tilde{D}_{l} C^{i} \xi\right] D_{i}+\epsilon^{K L} X^{k}{ }_{, K} \tilde{D}_{k} C^{i} \xi D_{i, L}+C^{i} \xi \tilde{D}_{i}
\end{aligned}
$$

where $C \xi$ is the element of $\mathrm{f}_{\theta 1,0}{ }^{0}$ corresponding to $c \xi$ and is given explicitly by $C^{i} \xi=$ $c^{i} \xi+\theta^{K} x^{j}{ }_{, K} \partial_{x j} c^{i} \xi+\frac{1}{2} \epsilon_{K L} \theta^{K} \theta^{L}\left[\tilde{x}^{j} \partial_{x j} c^{i} \xi+\frac{1}{2} \epsilon^{M N} x^{j}{ }_{, M} x^{k}{ }_{, N} \partial_{x j} \partial_{x k} c^{i} \xi\right]$. By (7.2.6a), (7.2.8), $I(\xi), I_{, A}(\xi), \tilde{I}(\xi)$ are globally defined derivations on $\mathrm{f}_{\theta 0,0}$.

Using the relation $D_{i} C^{j} \xi=0, D_{i, A} C^{j} \xi=0$, it is now straightforward to verify that $H_{A}, H_{A, B}, \tilde{H}_{A}, I, I_{, A}, \tilde{I}$ satisfy relations (2.2.2a)-(2.2.2f), (2.2.6a)-(2.2.6f), (2.2.9a)(2.2.9i). In this way, $\mathrm{f}_{\theta 0,0}$ becomes a $\mathbb{Z}$ graded real left module algebra of the $\mathbb{Z}$ graded real Lie algebra $t_{\theta}($ cfr. sect 2.2$)$.

Thus, $\mathrm{f}:=\mathrm{f}_{00,0}$ acquires the structure of $N=2 \mathfrak{g}$ superoperation (cfr. def. 4.2.1), the relevant graded derivations being

$$
\begin{array}{ll}
h_{A} & =-x^{i}{ }_{, A} \tilde{\partial}_{x i}, \quad h_{A, B}=x^{i}{ }_{, A} \epsilon_{B L} \partial_{x i}{ }^{L}{ }^{L}-\epsilon_{A B} \tilde{x}^{i} \tilde{\partial}_{x i} \\
\tilde{h}_{A} & =\tilde{x}^{i} \epsilon_{A L} \partial_{x i}{ }^{L}-x^{i}{ }_{, A} \partial_{x i} .
\end{array}
$$




$$
\begin{aligned}
& i(\xi)=c^{i} \xi \tilde{\partial}_{x i}, \quad i_{, A}(\xi)=c^{i} \xi \epsilon_{A L} \partial_{x i}{ }^{L}+x^{j}{ }_{, A} \partial_{x j} c^{i} \xi \tilde{\partial}_{x i}, \\
& \tilde{\imath}(\xi)=c^{i} \xi \partial_{x i}+x^{j}{ }_{, K} \partial_{x j} c^{i} \xi \partial_{x i}, K+\left[\tilde{x}^{j} \partial_{x j} c^{i} \xi+\frac{1}{2} \epsilon^{K L} x^{k}{ }_{, K} x^{l}{ }_{, L} \partial_{x k} \partial_{x l} c^{i} \xi\right] \tilde{\partial}_{x i} .
\end{aligned}
$$

This superoperation is canonically associated to the $N=2$ differential structure.

In spite of the fact that, in the $N=2$ case, $f$ does not have any simple geometrical interpretation, unlike its $N=1$ counterpart, the (basic) cohomology of $\mathrm{f}$ in the $N=2$ case has essentially the same content as that of the $N=1$ case and a theorem analogous to theor. 7.1.1 holds.

Theorem 7.2.1. There is an isomorphism of the $N=2$ (basic) cohomology of $\mathrm{f}$ the de Rham (basic) cohomology of the $(G)$ manifold $M$. Indeed, one has that $H^{n, p}(\mathrm{f})=0$ $\left(H_{\text {basic }}^{n, p}(\mathfrak{f})=0\right)$, except perhaps for $(n, p)=(1,0),(r, r+1)$ with $1 \leq r \leq m$, and

$$
\begin{gathered}
H^{1,0}(\mathfrak{f}) \simeq H_{\mathrm{dR}}^{0}(M), \quad H^{r, r+1}(\mathfrak{f}) \simeq H_{\mathrm{dR}}^{r}(M) \otimes \bigvee^{r-1} \mathbb{R}^{2}, \quad 1 \leq r \leq m, \quad(7.2 .13 a),(7.2 .13 \\
H_{\text {basic }}^{1,0}(\mathrm{f}) \simeq H_{\mathrm{dR} \text { basic }}^{0}(M), \quad H_{\text {basic }}^{r, r+1}(\mathfrak{f}) \simeq H_{\mathrm{dR} \text { basic }}^{r}(M) \otimes \bigvee^{r-1} \mathbb{R}^{2}, \quad 1 \leq r \leq m .
\end{gathered}
$$

Proof. By prop. 4.2.2, $H^{n, p}(\mathbf{f})=0\left(H_{\text {basic }}^{n, p}(\mathfrak{f})=0\right)$ except perhaps for $p= \pm n+1$. On the other hand, from the definition of $\mathrm{f}$, given above, $\mathrm{f}^{n, p}=0$ for $p<0$. So, $H^{n, p}(\mathrm{f})=0$ $\left(H_{\text {basic }}^{n, p}(\mathfrak{f})=0\right)$ except perhaps for $(n, p)=(1,0),(r, r+1)$ with $1 \leq r$. Consider first the case where $(n, p)=(1,0)$. From $(7.2 .9 \mathrm{~b})$ and the representation theory of $\mathbf{i}=\mathfrak{s l}(2, \mathbb{R}) \oplus \mathbb{R}$, it is immediate to see that $\mathrm{f}^{1,0}$ consists precisely of the $F$ of the form $F=\alpha$ for some smooth function $\alpha$ on $M$ and that $\mathrm{f}^{1,-2}=0$. Further, the conditions $d_{A} F=0$ is equivalent to $d_{\mathrm{dR}} \alpha=0$, hence to the local constance of $\alpha$. We thus have a linear bijection $\nu$ : $\mathrm{f}^{1,0} \cap \cap_{A=1,2} \operatorname{ker} d_{A} \mapsto Z_{\mathrm{dR}}^{0}(M)$, where $Z_{\mathrm{dR}}^{r}(M)$ is the space of closed $r$ forms, given by $F \mapsto \alpha$. Being $\mathrm{f}^{1,-2}=0,(7.2 .13 \mathrm{a})$ follows. (7.2.14a) also holds, as, clearly, $\mathrm{f}^{1,0}=\mathrm{f}_{\text {basic }}^{1,0}$ and $Z_{\mathrm{dR}}^{0}(M)=Z_{\mathrm{dR} \text { basic }}^{0}(M)$. Consider next the case where $(n, p)=(r, r+1)$ with $1 \leq r$. Let $F \in \mathrm{f}^{r, r+1}$. From $(7.2 .9 \mathrm{~b})$ and the representation theory of $\mathrm{i}=\mathfrak{s l}(2, \mathbb{R}) \oplus \mathbb{R}, F$ is locally of the form

$$
F=x^{i_{1}}{ }_{, A_{1}} \cdots x^{i_{r-1}}{ }_{, A_{r-1}}\left[\tilde{x}^{i_{r}} \alpha_{i_{1} \cdots i_{r-1} i_{r}}^{A_{1} \cdots A_{r-1}}+\frac{1}{2} \epsilon^{M N} x^{i_{r}}{ }_{M} x^{i_{r+1}}{ }_{, N} \beta_{i_{1} \cdots i_{r-1} i_{r} i_{r+1}}^{A_{1} \cdots A_{r-1}}\right]
$$

with $\alpha_{i_{1} \cdots i_{r-1} i_{r}}^{A_{1} \cdots A_{r-1}}$ a realvalued smooth map symmetric in $A_{1}, \cdots, A_{r-1}$ and antisymmetric in $i_{1}, \cdots, i_{r-1}$ and $\beta_{i_{1} \cdots i_{r-1} i_{r} i_{r+1}}^{A_{1} \cdots A_{i_{-1}}}$ a realvalued smooth map symmetric in $A_{1}, \cdots, A_{r-1}$, antisymmetric in $i_{1}, \cdots, i_{r-1}$ and symmetric in $i_{r}, i_{r+1}$. Next, assume that $d_{A} F=0$. 
Substituting (7.2.15) into the relation $d_{A} F_{A_{1} \cdots A_{r-1}}=0$ and taking into account the fact that terms with different numbers of $x^{i}{ }_{, I}, \tilde{x}^{i}$ are linearly independent and, thus, must vanish separately, one gets the following three identities

$$
\begin{aligned}
& x^{i_{1}}{ }_{, A_{1}} \cdots x^{i_{r-2}}{ }_{, A_{r-2}} \tilde{x}^{i_{r-1}} \tilde{x}^{i_{r}} \alpha_{i_{1} \cdots i_{r-1} i_{r}}^{A_{1} \cdots A_{r-1}}=0, \\
& (r-1) \epsilon_{A A_{r-1}} x^{i_{1}}{ }_{, A_{1}} \cdots x^{i_{r-2}}{ }_{, A_{r-2}} \frac{1}{2} \epsilon^{M N} x^{i_{r}}, M x^{i_{r+1}}{ }_{, N} \tilde{x}^{i_{r-1}} \beta_{i_{1} \cdots i_{r-1} i_{r} i_{r+1}}^{A_{1} \cdots A_{r-1}} \\
& +x^{i_{1}}{ }_{, A_{1}} \cdots x^{i_{r-1}}{ }_{, A_{r-1}}\left[-x^{i_{r}}{ }_{, A} \tilde{x}^{i_{r+1}} \beta_{i_{1} \cdots i_{r-1} i_{r} i_{r+1}}^{A_{1} \cdots A_{r-1}}+x^{i_{r+1}}{ }_{, A} \tilde{x}^{i_{r}} \partial_{x i_{r+1}} \alpha_{i_{1} \cdots i_{r-1} i_{r}}^{A_{1} \cdots A_{r-1}}\right]=0, \\
& x^{i_{1}}{ }_{, A_{1}} \cdots x^{i_{r-1}}{ }_{, A_{r-1}} \frac{1}{2} \epsilon^{M N} x^{i_{r}}{ }_{, M} x^{i_{r+1}}{ }_{, N} x^{i_{r+2}}{ }_{, A} \partial_{x i_{r+2}} \beta_{i_{1} \cdots i_{r-1} i_{r} i_{r+1}}^{A_{1} \cdots A_{r-1}}=0 .
\end{aligned}
$$

From (7.2.16a), using the symmetry properties of $\alpha_{i_{1} \cdots i_{r-1} i_{r}}^{A_{1} \cdots A_{r-1}}$ and the fact that $x^{i}, A, \tilde{x}^{i}$ are odd, even, respectively, it follows immediately that $\alpha_{i_{1} \cdots i_{r-2} i_{r-1} i_{r}}^{A_{1} \cdots A_{r-1}}+\alpha_{i_{1} \cdots i_{r-2} i_{r} i_{r-1}}^{A_{1} \cdots A_{r-1}}=0$. Since $\alpha_{i_{1} \cdots i_{r-1} i_{r}}^{A_{1} \cdots A_{r-1}}$ is already antisymmetric in $i_{1}, \cdots, i_{r-1}, \alpha_{i_{1} \cdots i_{r}}^{A_{1} \cdots A_{r-1}}$ is antisymmetric in all the indices $i_{1}, \cdots, i_{r}$. Thus, for fixed $A_{1}, \cdots, A_{r-1}$, the $\alpha_{i_{1} \cdots i_{r}}^{A_{1} \cdots A_{r-1}}$ are the coefficients of a local $r$ form $\alpha^{A_{1} \cdots A_{r-1}}$. Next, applying the derivation $u_{B}$ (cfr. eq. (7.2.11a)) to eq. (7.2.16b) and contracting with $\epsilon^{B A}$, one gets

$$
\begin{aligned}
& x^{i_{1}}{ }_{, A_{1}} \cdots x^{i_{r-1}}, A_{r-1} \frac{1}{2} \epsilon^{M N} x^{i_{r}}{ }_{, M} x^{i_{r+1}}{ }_{, N} \beta_{i_{1} \cdots i_{r-1} i_{r} i_{r+1}}^{A_{1} \cdots A_{r-1}} \\
& =\frac{2}{r+1} x^{i_{1}}{ }_{, A_{1}} \cdots x^{i_{r-1}}{ }_{, A_{r-1}} \frac{1}{2} \epsilon^{M N} x^{i_{r}}, M x^{i_{r+1}}{ }_{, N} \partial_{x i_{r+1}} \alpha_{i_{1} \cdots i_{r-1} i_{r}}^{A_{1} \cdots A_{r-1}}
\end{aligned}
$$

Applying $d_{A}$ to this relation, one gets

$$
\begin{aligned}
& (r-1) \epsilon_{A A_{r-1}} x^{i_{1}}{ }_{, A_{1}} \cdots x^{i_{r-2}}{ }_{, A_{r-2}} \frac{1}{2} \epsilon^{M N} x^{i_{r}}, M x^{i_{r+1}}{ }_{, N} \tilde{x}^{i_{r-1}} \beta_{i_{1} \cdots i_{r-1} i_{r} i_{r+1}}^{A_{1} \cdots A_{r-1}} \\
& -x^{i_{1}}{ }_{, A_{1}} \cdots x^{i_{r-1}}{ }_{, A_{r-1}} x^{i_{r}}{ }_{, A} \tilde{x}^{i_{r+1}} \beta_{i_{1} \cdots i_{r-1} i_{r} i_{r+1}}^{A_{1} \cdots A_{r-1}} \\
& =2 \frac{r-1}{r+1} \epsilon_{A A_{r-1}} x^{i_{1}}{ }_{, A_{1}} \cdots x^{i_{r-2}}{ }_{, A_{r-2}} \frac{1}{2} \epsilon^{M N} x^{i_{r}}, M x^{i_{r+1}}{ }_{, N} \tilde{x}^{i_{r-1}} \partial_{x i_{r+1}} \alpha_{i_{1} \cdots i_{r-1} i_{r}}^{A_{1} \cdots A_{r-1}} \\
& -\frac{1}{r+1} x^{i_{1}}{ }_{, A_{1}} \cdots x^{i_{r-1}}{ }_{, A_{r-1}} x^{i_{r}}{ }_{, A} \tilde{x}^{i_{r+1}}\left(\partial_{x i_{r+1}} \alpha_{i_{1} \cdots i_{r-1} i_{r}}^{A_{1} \cdots A_{r-1}}+\partial_{x i_{r}} \alpha_{i_{1} \cdots i_{r-1} i_{r+1}}^{A_{1} \cdots A_{r-1}}\right) \text {, } \\
& x^{i_{1}}{ }_{, A_{1}} \cdots x^{i_{r-1}}{ }_{, A_{r-1}} \frac{1}{2} \epsilon^{M N} x^{i_{r}}, M x^{i_{r+1}}{ }_{, N} x^{i_{r+2}}{ }_{, A} \partial_{x i_{r+2}} \beta_{i_{1} \cdots i_{r-1} i_{r} i_{r+1}}^{A_{1} \cdots A_{r-1}} \\
& =\frac{2}{r+1} x^{i_{1}}{ }_{, A_{1}} \cdots x^{i_{r-1}}{ }_{, A_{r-1}} \frac{1}{2} \epsilon^{M N} x^{i_{r}}{ }_{, M} x^{i_{r+1}}{ }_{, N} x^{i_{r+2}}{ }_{, A} \partial_{x i_{r+1}} \partial_{x i_{r+2}} \alpha_{i_{1} \cdots i_{r-1} i_{r}}^{A_{1} \cdots A_{r-1}} .
\end{aligned}
$$


Substituting (7.2.18a), (7.2.18b) into (7.2.16b), (7.2.16c), respectively, one obtains after a straightforward calculation the equations

$$
\begin{aligned}
& x^{i_{1}}{ }_{, A_{1}} \cdots x^{i_{r-1}}{ }_{, A_{r-1}} x^{i_{r+1}}{ }_{, A_{r+1}} \tilde{x}^{i_{r}} \sum_{l=1}^{r+1}(-1)^{l-1} \partial_{x i_{l}} \alpha_{i_{1} \cdots i_{l-1} i_{l+1} \cdots i_{r+1}}^{A_{1} \cdots A_{r-1}}=0 \\
& x^{i_{1}}{ }_{, A_{1}} \cdots x^{i_{r-1}}{ }_{, A_{r-1}} \frac{1}{2} \epsilon^{M N} x^{i_{r}}, M x^{i_{r+1}}{ }_{, N} x^{i_{r+2}}{ }_{, A} \partial_{x i_{r+1}} \partial_{x i_{r+2}} \alpha_{i_{1} \cdots i_{r-1} i_{r}}^{A_{1} \cdots A_{r-1}}=0 .
\end{aligned}
$$

Using the symmetry properties of $\alpha_{i_{1} \cdots i_{r-1} i_{r}}^{A_{1} \cdots A_{r-1}}$ and the fact that $x^{i}, A, \tilde{x}^{i}$ are odd, even, respectively, it is easy to see, that (7.2.19a) implies that $\sum_{l=1}^{r+1}(-1)^{l-1} \partial_{x i_{l}} \alpha_{i_{1} \cdots i_{l-1} i_{l+1} \cdots i_{r+1}}^{A_{1} \cdots A_{r-1}}=0$ or $d_{\mathrm{dR}} \alpha^{A_{1} \cdots A_{r-1}}=0$ so that the local $r$ form $\alpha^{A_{1} \cdots A_{r-1}}$ is closed and locally exact. By this reason and the fact that $x^{i}{ }_{,} \partial_{x i} x^{j}{ }_{,} \partial_{x j} x^{k}{ }_{, K} \partial_{x k}=0$ by antisymmetry, one finds that eq. (7.2.19b) is automatically satisfied. We note that, by (7.2.5a) and the global definition of $F$, the local exact $r$ form $\alpha^{A_{1} \cdots A_{r-1}}$ is the local restriction of a globally defined closed $r$ form, which will be denoted by the same symbol. To summarize, we have shown that (7.2.16a)-(7.2.16c) imply that, for fixed $A_{1}, \cdots, A_{r-1}, \alpha^{A_{1} \cdots A_{r-1}}$ is a closed $r$ form and that (7.2.17) holds. Conversely, assume that for fixed $A_{1}, \cdots, A_{r-1}, \alpha^{A_{1} \cdots A_{r-1}}$ is a closed $r$ form and that (7.2.17) holds. Using (7.2.5a), (7.2.5b), it is straightforward though tedious to show that $F$, as given by (7.2.15), belongs to $f^{r, r+1}$. As shown above, (7.2.17) implies (7.2.18a), (7.2.18b) using which eqs. (7.2.16b), (7.2.16c) become equivalent to eqs. (7.2.19a), (7.2.19b). Eqs. (7.2.16a), (7.2.19a), (7.2.19b), are trivially satisfied by the closed $r$ form $\alpha^{A_{1} \cdots A_{r-1}}$. Thus, (7.2.16a)-(7.2.16c) are satisfied as well implying that $d_{A} F=0$. In conclusion, we have shown that $\mathrm{f}^{r, r+1} \cap \cap_{A=1,2}$ ker $\left.d\right)_{A}$ consists precisely of the elements $F \in \mathrm{f}^{r, r+1}$ of the form

$$
F=x^{i_{1}}{ }_{, A_{1}} \cdots x^{i_{r-1}}{ }_{, A_{r-1}}\left[\tilde{x}^{i_{r}} \alpha_{i_{1} \cdots i_{r-1} i_{r}}^{A_{1} \cdots A_{r-1}}+\frac{2}{r+1} \frac{1}{2} \epsilon^{M N} x^{i_{r}}{ }_{, M} x^{i_{r+1}}{ }_{, N} \partial_{x i_{r+1}} \alpha_{i_{1} \cdots i_{r-1} i_{r}}^{A_{1} \cdots A_{r-1}}\right]
$$

with $\alpha^{A_{1} \cdots A_{r-1}}$ an $r$ form symmetric in $A_{1}, \cdots, A_{r-1}$ and such that $d_{\mathrm{dR}} \alpha^{A_{1} \cdots A_{r-1}}=0$. We thus have a linear bijection $\nu: \mathrm{f}^{r, r+1} \cap \cap_{A=1,2} \operatorname{ker} d_{A} \mapsto Z_{\mathrm{dR}}^{r}(M) \otimes \bigvee^{r-1} \mathbb{R}^{2}$, where $Z_{\mathrm{dR}}^{r}(M)$ is the space of closed $r$ forms, given by $F \mapsto\left(\alpha^{A_{1} \cdots A_{r-1}}\right)_{A_{1}, \cdots, A_{r-1}=1,2}$. Next, assume that $F \in \frac{1}{2} \epsilon^{K L} d_{K} d_{L} \mathrm{f}^{r, r-1}$. Then, $F=\frac{1}{2} \epsilon^{K L} d_{K} d_{L} G$ for some $G \in \mathrm{f}^{r, r-1}$. From (7.2.9b) and the representation theory of $\mathrm{i}=\mathfrak{s l}(2, \mathbb{R}) \oplus \mathbb{R}, G$ is of the form

$$
G=x^{i_{1}}{ }_{, A_{1}} \cdots x^{i_{r-1}}, A_{r-1} \gamma_{i_{1} \cdots i_{r-1}}^{A_{1} \cdots A_{r-1}}
$$

with $\gamma_{i_{1} \cdots i_{r-1}}^{A_{1} \cdots A_{r-1}}$ a realvalued smooth map symmetric in $A_{1}, \cdots, A_{r-1}$ and antisymmetric in $i_{1}, \cdots, i_{r-1}$. By a straightforward computation, one finds that

$$
\begin{gathered}
\frac{1}{2} \epsilon^{K L} d_{K} d_{L} G=(-1)^{r-1} x^{i_{1}}{ }_{, A_{1}} \cdots x^{i_{r-1}}{ }_{, A_{r-1}}\left[\tilde{x}^{i_{r}} \sum_{l=1}^{r}(-1)^{l-1} \partial_{x i_{l}} \gamma_{i_{1} \cdots i_{l-1} i_{l+1} \cdots i_{r-1} i_{r}}^{A_{1} \cdots A_{r-1}}\right. \\
\left.+\frac{2}{r+1} \frac{1}{2} \epsilon^{M N} x_{, M}^{i_{r}} x^{i_{r+1}}{ }_{, N} \partial_{x i_{r+1}} \sum_{l=1}^{r}(-1)^{l-1} \partial_{x i_{l}} \gamma_{i_{1} \cdots i_{l-1} i_{l+1} \cdots i_{r-1} i_{r}}^{A_{1} \cdots A_{r-1}}\right]
\end{gathered}
$$


Note that the $\gamma_{i_{1} \cdots i_{r-1}}^{A_{1} \cdots A_{r-1}}$ are the coefficients of a local $r-1$ form $\gamma^{A_{1} \cdots A_{r-1}}$. By (7.2.5a) and the global definition of $G, \gamma^{A_{1} \cdots A_{r-1}}$ is the restriction of a globally defined $r-1$ form, which we shall denote by the same symbol. As (7.2.22) indicates, the linear map $\nu$ maps cohomologically trivial elements of $\mathrm{f}^{r, r+1} \cap \cap_{A=1,2} \operatorname{ker} d_{A}$ into cohomologically trivial elements of $Z_{\mathrm{dR}}^{r}(M) \otimes \bigvee^{r-1} \mathbb{R}^{2}$. Thus, $\nu$ induces a linear bijection $\hat{\nu}: H^{r, r+1}(\mathrm{f}) \mapsto H_{\mathrm{dR}}^{r}(M) \otimes \bigvee^{r-1} \mathbb{R}^{2}$. Next, assume that $F \in \mathrm{f}_{\text {basic }}^{r, r+1}$ and that $d_{A} F=0$. In particular, $F$ is of the form (7.2.20) for some closed $r$ form $\alpha^{A_{1} \cdots A_{r-1}}$ symmetric in $A_{1}, \cdots, A_{r-1}$. By $(2.2 .19 \mathrm{~d}),(2.2 .19 \mathrm{e})$ and the relation $d_{A} F=0$, the basicity of $F$ is equivalent to the relation $j(\xi) F=0, \xi \in \mathfrak{g}$, where $j(\xi)$, by $(2.2 .15 \mathrm{a})$, is given in the present situation by (7.2.12a). A simple computation shows that this identity is equivalent to

$$
x^{i_{1}}{ }_{, A_{1}} \cdots x^{i_{r-1}}, A_{r-1} c^{i_{r}} \xi \alpha_{i_{1} \cdots i_{r-1} i_{r}}^{A_{1} \cdots A_{r-1}}=0 .
$$

As is straightforward to check, this relation entails that $c^{i_{0}} \xi \alpha_{i_{0} i_{1} \cdots i_{r-1}}^{A_{1} \cdots A_{r-1}}=0$, so that $j_{\mathrm{dR}}(\xi) \alpha^{A_{1} \cdots A_{r-1}}=0$. As $l_{\mathrm{dR}}(\xi)=\left[d_{\mathrm{dR}}, j_{\mathrm{dR}}(\xi)\right]$ and $d_{\mathrm{dR}} \alpha^{A_{1} \cdots A_{r-1}}=0$, the closed $r$ form $\alpha^{A_{1} \cdots A_{r-1}}$ is basic. Conversely, if $\alpha^{A_{1} \cdots A_{r-1}}$ is basic (7.2.23) obviously holds. So, the linear bijection $\nu$ introduced earlier maps $\mathrm{f}_{\text {basic }}^{r, r+1} \cap \cap_{A=1,2} \operatorname{ker} d_{A}$ into $Z_{\mathrm{dR} \text { basic }}^{r}(M) \otimes \bigvee^{r-1} \mathbb{R}^{2}$, where $Z_{\mathrm{dR} \text { basic }}^{r}(M)$ is the space of closed basic $r$ forms. Let $G \in \mathrm{f}_{\mathrm{basic}}^{r, r-1}$. Then, $G$ is of the form (7.2.21) and satisfies $j(\xi) G=0, j_{A}(\xi) G=0, l(\xi) G=0$, where $j(\xi), j_{A}(\xi)$ and $l(\xi)$ are defined by $(2.2 .15 \mathrm{a})-(2.2 .15 \mathrm{c})$ and are given by $(7.2 .12 \mathrm{a})-(7.2 .12 \mathrm{c})$. It is straightforward to see that these identities yields the equations

$$
\begin{aligned}
& \epsilon_{A A_{r-1}} x^{i_{1}}{ }_{, A_{1}} \cdots x^{i_{r-2}}, A_{r-2} c^{i_{r-1}} \xi \gamma_{i_{1} \cdots i_{r-1}}^{A_{1} \cdots A_{r-1}}=0, \\
& x^{i_{1}}{ }_{, A_{1}} \cdots x^{i_{r-1}}, A_{r-1}\left[\sum_{l=1}^{r-1} \partial_{x i_{l}} c^{i_{r}} \xi \gamma_{i_{1} \cdots i_{l-1} i_{r} i_{l+1} \cdots i_{r-1}}^{A_{1} \cdots A_{r-1}}+c^{i_{r}} \xi \partial_{x i_{r}} \gamma_{i_{1} \cdots i_{r-1}}^{A_{1} \cdots A_{r-1}}\right]=0 .
\end{aligned}
$$

Thus, $c^{i_{0}} \xi \gamma_{i_{0} i_{1} \cdots i_{r-2}}^{A_{1} \cdots A_{r-1}}=0, \sum_{l=1}^{r-1} \partial_{x i_{l}} c^{i_{r}} \xi \gamma_{i_{1} \cdots i_{l-1} i_{r} i_{l+1} \cdots i_{r-1}}^{A_{1} \cdots A_{r-1}}+c^{i_{r}} \xi \partial_{x i_{r}} \gamma_{i_{1} \cdots i_{r-1}}^{A_{1} \cdots A_{r-1}}=0$, as is easy to see, so that $j_{\mathrm{dR}}(\xi) \gamma^{A_{1} \cdots A_{r-1}}=0$ and $l_{\mathrm{dR}}(\xi) \gamma^{A_{1} \cdots A_{r-1}}=0$ and $\gamma^{A_{1} \cdots A_{r-1}}$ is basic. Conversely the basicity of $\gamma^{A_{1} \cdots A_{r-1}}$ implies (7.2.24a), (7.2.24b). From (7.2.21), (7.2.22), we see that $\nu$ maps cohomologically trivial elements of $\mathrm{f}_{\text {basic }}^{r, r+1} \cap \cap_{A=1,2} \operatorname{ker} d_{A}$ into cohomologically trivial elements of $Z_{\mathrm{dR} \text { basic }}^{r}(M) \otimes \bigvee^{r-1} \mathbb{R}^{2}$. Thus, $\nu$ induces a linear bijection $\hat{\nu}: H_{\text {basic }}^{r, r+1}(\mathrm{f}) \mapsto H_{\mathrm{dR} \text { basic }}^{r}(M) \otimes \bigvee^{r-1} \mathbb{R}^{2}$.

A theorem analogous to theor. 7.1.2 also holds.

Theorem 7.2.2. One has

$$
\operatorname{Conn}(\mathrm{f}) \simeq \operatorname{Conn}(M)
$$

(cfr. def. 6.2.1). 
Proof. From the representation theory of $\mathrm{i}=\mathfrak{s l}(2, \mathbb{R}) \oplus \mathbb{R}$, any $a_{A} \in \mathfrak{f}^{2,1} \otimes \mathfrak{g}$ is locally of the form $a_{A}=a_{i} \tilde{x}^{i}{ }_{A}$, where $a_{i}$ is a $\mathfrak{g}$ valued smooth map. Define $\lambda\left(\left(a_{A}\right)_{A=1,2}\right)=a_{i} d_{\mathrm{dR}} x^{i}$. Then, from (7.2.12a) $-(7.2 .12 \mathrm{c})$, it is easy to see that $\lambda\left(\left(a_{A}\right)_{A=1,2}\right)$ is a connection of $M$ if and only if $\left(a_{A}\right)_{A=1,2}$ is a connection of $\mathrm{f}$. The map $\lambda$ is clearly a bijection.

7.3. The relation between the $N=1$ and $N=2$ cohomologies of $\mathrm{f}$

Let $\mathrm{f}(n)$ denote the superoperation $\mathrm{f}$ for $N=n, n=1,2$, as defined in subsects. 7.1, 7.2 .

Corollary 7.3.1. One has

$$
\begin{aligned}
& H^{n, \pm n+1}(\mathrm{f}(2)) \simeq H^{ \pm\left(n-\frac{1}{2}\right)+\frac{1}{2}}(\mathrm{f}(1)) \otimes \bigvee^{n-1} \mathbb{R}^{2} \\
& H_{\text {basic }}^{n, \pm n+1}(\mathrm{f}(2)) \simeq H_{\text {basic }}^{ \pm\left(n-\frac{1}{2}\right)+\frac{1}{2}}(\mathrm{f}(1)) \otimes \bigvee^{n-1} \mathbb{R}^{2} .
\end{aligned}
$$

Proof. Combine props. 7.1.1, 7.2.1.

Thus, the $N=1$ and $N=2$ cohomologies of $\mathrm{f}$ are closely related. Note the analogy to relations (5.3.1), (5.3.2).

Corollary 7.3.2. One has

$$
\operatorname{Conn}(\mathrm{f}(2)) \simeq \operatorname{Conn}(\mathrm{f}(1))
$$

Proof. Combine props. 7.1.2, 7.2.2.

Thus, the $N=1$ and $N=2$ connections of $f$ are manifestations of the same geometrical structure.

\section{Concluding remarks}

There are a few fundamental questions which are still open and which are of considerable salience both in geometry and topological field theory.

Cors. 5.3.1, 7.3.1 suggest that a relation formally analogous to (7.3.2) should hold also between the $N=1$ and $N=2$ equivariant cohomologies of $\mathrm{f}$ (cfr. sect. 6). Further, from (7.3.3), we expect that the range of the $N=1$ and $N=2$ Weil homomorphisms (cfr. subsects. 6.1, 6.2) should have essentially the same content. This question is of fundamental importance to show conclusively that balanced topological gauge field theory does not contain new topological observables besides those coming from the underlying $N=1$ theory. We have not been able to either proove or disproove such assertions yet.

There are other possible lines of inquiry. It is known that the $N=1$ Maurer-Cartan equations of a Lie algebra $\mathfrak{g}$ can be obtained from the $N=1$ Weil algebra relation (3.1.11a) 
by formally setting $\phi=0$. By a similar procedure, one can obtain the $N=2$ MaurerCartan equations by formally setting $\phi_{A B}=0, \rho_{A}=0$ in the $N=2$ Weil algebra relations $(3.2 .11 \mathrm{e}),(3.2 .11 \mathrm{~g})$. Indeed, it is straigtforward to check that the basic relation $\left[d_{A}, d_{B}\right]=0$ still holds after this truncation. This hints to a possible $N=2$ generalization of gauge fixing.

Finally, note that, by obtaining the $N=2$ Weil algebra, we are in the position of formulating other models of equivariant cohomology in balanced topological field theory besides Cartan's used in [31], generalizing the $N=1$ intermediate or BRST model of [7,8].

We leave these matters to future work [34].

Acknowledgements. We are greatly indebt to R. Stora for providing his invaluable experience and relevant literature.

\section{REFERENCES}

[1] S. Cordes, G. Moore and S. Ramgoolam, Lectures on 2-D Yang-Mills Theory, Equivariant Cohomology and Topological Field Theories, presented at the 1994 Trieste Spring School on String Theory, Gauge Theory and Quantum Gravity, Trieste, Italy, 11 April - 22 April 1994, and at the NATO Advanced Study Institute, Les Houches Summer School, Session 62: Fluctuating Geometries in Statistical Mechanics and Field Theory, Les Houches, France, 2 August - 9 September 1994, Nucl. Phys. Proc. Suppl. 41 (1995), 184, and Les Houches proceedings, hep-th/9411210.

[2] R. Dijkgraaf, Les Houches Lectures on Fields, Strings and Duality, lectures given at NATO Advanced Study Institute at the Les Houches Summer School on Theoretical Physics, Session 64: Quantum Symmetries, Les Houches, France, 1 August - 8 September 1995, hep-th/9703136.

[3] J. M. F. Labastida and C. Lozano, Lectures in Topological Quantum Field Theory, talk given at La Plata Meeting on Trends in Theoretical Physics, La Plata, Argentina, 28 April - 6 May 1997, CERN-TH-97-250, hep-th/9709192.

[4] E. Witten, Introduction to Cohomological Field Theories, Lectures at the Trieste Workshop on Topological Methods in Physics, Trieste, Italy, June 1990, Int. J. Mod. Phys. A6 (1991), 2775.

[5] L. Baulieu and I. M. Singer, Topological Yang-Mills Symmetry, Nucl. Phys. Proc. Suppl. 5B (1988), 12.

[6] L. Baulieu and I. M. Singer, Conformally Invariant Gauge Fixed Actions for 2-D Topological Gravity, Commun. Math. Phys. 135 (1991), 253. 
[7] J. Kalkman, BRST Model for Equivariant Cohomology and Representatives for the Equivariant Thom Class, Commun. Math. Phys. 153 (1993), 447.

[8] S. Ouvry, R. Stora and P. van Baal, On the Algebraic Characterization of Witten Topological Yang-Mills Theory, Phys. Lett. B220 (1989), 159.

[9] R. Stora, F. Thuillier and J.-C. Wallet, Algebraic Structure of Cohomological Field Theory Models and Equivariant Cohomology, lectures presented at the First Carribean School of Mathematics and Theoretical Physics, Saint Francois, Guadaloupe MayJune 1993.

[10] V. Mathai and D. Quillen, Superconnections, Thom Classes and Equivariant Differential Forms, Topology 25 (1986), 85.

[11] M. F. Atiyah and L. Jeffrey, Topological Lagrangians and Cohomology, J. Geom. Phys. 7 (1990), 119.

[12] M. Blau, The Mathai-Quillen Formalism and Topological Field Theory, J. Geom. Phys. 11 (1991), 129.

[13] R. Stora, Equivariant Cohomology and Topological Field Theories, talk given at the International Symposium on BRS Symmetry on the Occasion of its 20-th Anniversary, Kyoto Japan, 18 September - 22 September 1995, ENSLAPP-A-571-95; Exercises in Equivariant Cohomology, talk given at NATO Advanced Study Institute on Quantum Fields and Quantum Space Time, Cargese, France, 22 July - 3 August 1996. in Cargese 1996, Quantum Fields and Quantum Space Time, 265, hep-th/9611114; Exercises in Equivariant Cohomology and Topological Field Theories, talk given at Symposium on the Mathematical Beauty of Physics, Gif-sur-Yvette, France, 5 June 7 June 1996, in Saclay 1996, The Mathematical Beauty of Physics, 51, hep-th/9611116.

[14] J. P. Yamron, Topological Actions for Twisted Supersymmetric Theories, Phys. Lett. B213 (1988), 325.

[15] E. Witten, Topology Changing Amplitudes in $2+1$ Dimensional Gravity, Nucl. Phys. B323 (1989), 113.

[16] D. Birmingham, M. Blau and G. Thompson, Geometry and Quantization of Topological Gauge Theories, Int. J. Mod. Phys. A5 (1990), 4721.

[17] M. Blau and G. Thompson, N=2 Topological Gauge Theory, the Euler Characteristic of Moduli Spaces and the Casson Invariant, Commun. Math. Phys. 152 (1993), 41, hep-th/9112012.

[18] C. Vafa and E. Witten, A Strong Coupling Test of S Duality, Nucl. Phys. B431 (1994), 3, hep-th/9408074.

[19] M. Bershadsky, A. Johansen, V. Sadov and C. Vafa, Topological Reduction of 4-D SYM to 2-D Sigma Models, Nucl. Phys. B448 (1995), 166, hep-th/9501096. 
[20] N. Marcus, The Other Topological Twisting of $N=4$ Yang-Mills Nucl. Phys. B452 (1995), 331, hep-th/9506002.

[21] M. Blau and G. Thompson, Aspects of $N_{T} \geq 2$ Topological Gauge Theories and DBranes, Nucl. Phys. B492 (1997), 545, hep-th/9612143.

[22] J. M. F. Labastida and C. Lozano, Mathai-Quillen Formulation of Twisted $\mathrm{N}_{4} 4 \mathrm{Su}$ persymmetric Gauge Theories in Four Dimensions, Nucl. Phys. B502 (1997), 741, hep-th/9702106.

[23] J. M. F. Labastida and C. Lozano, Mass Perturbations in Twisted N=4 Supersymmetric Gauge Theories, CERN-TH-97-316, hep-th/9711132.

[24] By R. Dijkgraaf, J. -S. Park and B. J. Schroers, N=4 Supersymmetric Yang-Mills Theory on a Kaehler Surface, ITFA-97-09, hep-th/9801066.

[25] M. Bershadsky, V. Sadov and C. Vafa, D-Branes and Topological Field Theories, Nucl. Phys. B463 (1996), 420, hep-th/9511222.

[26] M. Blau and G. Thompson, Euclidean SYM Theories by Time Reduction and Special Holonomy Manifolds Phys. Lett. B415 (1997), 242, hep-th/9706225.

[27] B. S. Acharya, J. M. Figueroa-O'Farrill, B. Spence and M. O'Loughlin, Euclidean D-Branes and Higher Dimensional Gauge Theory QMW-PH-97-20, hep-th/9707118.

[28] J. M. Figueroa-O'Farrill, A. Imaanpur and J. McCarthy, Supersymmetry and Gauge Theory on Calabi-Yau Three-Folds, QMW-PH-97-29, hep-th/9709178.

[29] J. -S. Park, Monads and D-Instantons, Nucl. Phys. B493 (1997), 198, hep-th/9612 096.

[30] C. Hofman and J. -S. Park, Monads, Strings, and M Theory, THU-97-14A, hepth/9706130.

[31] R. Dijkgraaf and G. Moore, Balanced Topological Field Theoreis, Commun. Math. Phys. 185 (1997), 411, hep-th/9608169.

[32] J. Manes, R. Stora and B. Zumino, Algebraic Study of Chiral Anomalies, Commun. Math. Phys. 102 (1985), 157.

[33] W. Grueb, S. Halperin and R. Vanstone, Connections, Curvature and Cohomology, vol. III, Academic Press, New York 1973

[34] R. Zucchini, im preparation. 